\title{
Towards a Policy for Bilingual education in Developing Countries
}

\author{
Christopher Stroud \\ University of the Western Cape and Stockholm University
}

\begin{abstract}
Executive summary
Language can make a difference to peoples' lives in many ways. On one dimension, language empowers by serving as a resource for individuals to constitute and trans-form social and personal identities and by providing access to important socio-economic and political markets. Language can empower because it does not merely reflect a pre-existing reality; it is itself - under certain conditions - an instrument in the constitution of these realities, by providing a new version of meaning that offers speakers a fresh interpretation or alternative perspective on reality. The multilingual proficiency that social elites have in important global languages such as English, French, German, and today, Chinese, is a case in point. These elites have long recognized that mastery of many languages is an economic asset to be cultivated and passed on to successive generations.
\end{abstract}

But just as commonly, language may also be a means by which speakers and communities are marginalized and disempowered. This happens when minority language communities are barred from access to important socio-economic institutions and markets on the grounds that they do not speak the appropriate languages for these activities, or when individuals are stigmatised and discriminated against in political and educational contexts because they do not master the official language used in these domains. These communities may host highly multilingual individuals, but the language portfolio or verbal repertoire they have at their disposal neither fit market demands nor the linguistic profile of those in power. This comparison alerts us to the fact that the notion of multilingualism comprises very many different types of language practice and perception, and that some of these practices are accorded a lesser value than others. In fact, we could see the verbal repertoire or set of multilingual practices that communities have access to as one way in which power and marginalisation are mediated and transmitted over generations. In this report, we are concerned precisely with marginalized multilingual indigenous communities, where linguistic practices mediate and help constitute disempowerment. I will be particularly concerned with exploring the ways in which formal educational institutions can either reaffirm community marginalisation or empower minority language speakers. In this context, I will detail a range of problems educationalists encounter when trying to provide bilingual or mother tongue education in minority languages. I will argue that, ultimately, the problems that these programmes confront are not educational problems per se, that is, they cannot be solved by educational means alone. Neither do they originate from the regrettably limited institutional support, commitment and resources most often provided for multilingual or mother tongue education. 
Rather, the well-nigh impossible task of creating well-functioning minority language programmes emanates from the nature of educational structures in global economies, whereby schools reinforce tendencies to marginality of minority languages and speakers already prevalent in outside society. Many of the problems that bilingual programmes are currently grappling with can be traced back to the relationship of former colonies to the colonial powers and to the existence of exploitative relation-ships within these societies between powerful and powerless social strata; the main problem that bilingual programmes encounter is "one of social structure" (cf. Robinson, 1996: 41). This suggests that a discussion of multilingual education should take place in a discourse of language and power, which would shift the terms of the debate away from an emphasis on the details of educational programmes to the more important, although also more elusive, framing conditions for multilingual education. In other words, in order to understand the nature of the problems in using indigenous languages in education, we need to understand the role played by multiple languages in mediating relationships of power between communities or social groups in contact. The report opens by exploring the many ways that minority languages have suffered throughout the course of colonialism, post colonialism and globalisation. As any policy needs to consider future language scenarios, this chapter concludes with a note on some possible effects of globalisation on language minority communities. Following on this, we shall look into some common language ideological debates or discourses on multilingualism and/or local languages. These debates occur in specific historical contexts and involve a struggle over definitions or representations of reality; "various representations of reality are pitted against each other - discursively - with the aim of gaining authority for one particular representation" (Blommaert, 1999: 9). These ideologies serve to mediate how speakers come to understand multilingualism in their lives.

The third set of concerns dealt with in this report are the ways in which social stigma is mediated institutionally in minority language contexts. How has mother tongue education been managed in schools throughout the developing world? And what are the factors that we need to refer to in order to account for this?

A central part of this report is the working through of an account of how minority groups' linguistic marginalisation is related to socio-economic and political marginalisation. The notion of a bivalent collectivity is introduced in this context. In Fraser's (1995) use of this term, it refers to groups where "neither socio-economic maldistribution or cultural misrecognition are an indirect effect of the other, but $[\ldots]$ both are primary and cooriginal". The conception of language and power articulated in this notion is used in this report to frame the set of policies and strategies, or remedies that educational language planners need to attend to in developing bilingual programmes. In essence, the claim I am making is that work on language issues must consistently go hand in hand with a more general socio-economic and political approach to the welfare of minority communities. The remaining sections of the report present a number of policy proposals that follow from this conception, as well as detail some points to consider in the implementation of these proposals. In conjunction with this, some ethical issues are dealt with that relate to what stance we may take on the issue of language loss and/or language shift. The main ethical issue revolves around the question of how to determine the choice(s) of minority languages in any specific national educational context. There will often be more languages around than available resources to teach them. Furthermore, the wishes of parents and local community may well not be in favour of using local languages as instructional media, even though this may go against the recommendations of expert discourses. Cases such as these are common, and require an ethical approach to a solution. 


\section{INTRODUCTION}

$\mathrm{I}_{\mathrm{c}}^{\mathrm{n}}$

1996, SIDA commissioned the Centre for Research on Bilingualism at Stockholm University to produce a report on the "State-of-the-Art" in bilingual and mother tongue education in developing countries. The brief was to formulate guidelines for future policy thinking in language education on the basis of an extensive review of published reports and evaluation; to develop a data base of studies; to establish contacts with institutions and organizations engaged in development work within the field of bilingual education; to produce an annotated bibliography; to write a report in English on factors found to impact upon the success or failure of bilingual and mother tongue programs; and to present the findings at a seminar. The Centre appointed Christopher Stroud to be responsible for designing and coordinating the project, as well as for writing up the final report. Three project assistants worked on putting together a dataset of mother tongue and bilingual programmes from various parts of the world; Margaret Obondo (countries where English is an official language), Christina Thornell (countries where French is an official language) and Birgitta Quick (countries where Portuguese and Spanish are official languages). Kerstin Simberg was the librarian responsible for compiling the bibliography.

In 1997, the team working on the project submitted three reports, Stroud, (1997); Stroud, Obondo, Quick and Thornell (1997); and Simberg (1997). A final report, with the working title $A n$ Overview and Analysis of Programmes was prefigured. The first report (Stroud, 1997), Bilingual Education in Developing Countries. I. Concepts, Models and Theories, has been extensively revised and is now part of the present report (more specifically, Chapters 1-8). The prefigured report comprises chapters 9 and 10 in the current policy text. In other words, the current volume entitled Towards a Policy for Bilingual Education in Developing Countries subsumes both the report on the theoretical background and the actual discussion and evaluation of existing programs. The data-set of programmes in bilingual and mother tongue education that have formed the empirical basis for this work, written up as Stroud, Obondo, Quick and Thornell (1997) is currently undergoing extensive reworking in preparation for its transformation into a web-based database.

Despite the fact that this report has been many years in the making, its conceptual formulation of the problems and issues embroiling bilingual and mother tongue programmes is still highly current. If anything, there is a growing awareness among an increasing number of people working with bilingual and mother tongue education that issues of language and education are fundamentally issues of power and marginalisation of minority language speakers in globally transformed economies. Likewise, the nature of the recommendations, emphasizing equity, social justice, democracy and citizenship, which force us to reconceptualise solutions to educational problems in nontraditional and non-educational terms of power and politics, is also gaining a wider acceptance - as evidenced by numerous recent publications (see, for example, the special issue of International Journal of Bilingualism and Bilingual Education, Vol. 2:3, 1999 on Indigenous Language Maintenance in Latin America). In his excellent review of politics and change in applied linguistics, Ben Rampton (1995) points out that contracted research 
and development work, of which the present report is an instance, suffer from some fairly obvious generic risks such as (a) limited time - sponsors often work to tight schedules, which restricts the amount of time an applied linguist has for finding out about the environment they are supposed to research. This predisposes the linguist towards choosing to work with models of language that might not be the best available or most appropriate, and it inhibits alternative data interpretations and impedes theoretical generalizations, as well as preventing attention to issues that may arise during and after the project, (b) overrapport with sponsors - researchers need to spend time negotiating the conduct of their work with sponsors, and there is a risk that research turns into personal management, public relations or advertising, (c) lack of academic context - the academic community often plays no significant role in the discussion of a project, which may mean less exposure to creative doubt and different theoretical accounts, and easily lead to "spurious exploitation of the prestige and credibility normally associated with independent re-search" (op. cit.: 242). Rampton goes on to say that

[c]ounterposed to these risks, however, there is often scope for applied linguists to negotiate the terms and conditions of their work, and this is increased if they are based in a supportive institution that allows them time to work and think sheltered from market pressures. (Rampton, 1995: 242)

To some extent, the conditions governing the production of the present report have been "negotiable". This has allowed time enough for its production, and permitted academic peer insight and commentary on the issues it raises, thereby offsetting some of the potential disadvantages with contracted research mentioned by Rampton. Especially important has been the network of contacts with institutions and departments working with language policy and education in various parts of the world, especially the Project for Alternative Education in South Africa (PRAESA) at the University of Cape Town headed by Dr. Neville Alexander, which have provided valuable insights on this project.

\section{TERMINOLOGICAL PRELIMINARIES}

The report uses various technical terms and concepts that need to be explained. Besides providing an explication of how these notions are used in the text, the chapter will illustrate how our beliefs about language fundamentally shape how we talk about it and work with it. The main focus of this chapter will be a discussion of 'standard language ideology' (cf. Lippi-Green). By this is meant "the bias towards an abstract, ideological, homogenous language which is imposed and maintained by dominant institutions, and which has as its model, the written language but which is drawn primarily from spoken language of the upper class" (2000). Here, I explore in a cursory fashion the implications that a standard language ideology has for the way in which we understand language, literacy, as well as first and second language acquisition. The chapter concludes with a discussion of language planning theory and practice, suggesting that language policies need to find ways of promoting 'ethnolinguistic' diversity. 


\section{Language}

In this report, different notions referring to language are used. A minority language is used in the sense of a less powerful language. That is, this terminology refers principally to the power relationship between languages, rather than to paucity. Official language refers to the language that the state has allotted to serve administrative, educational, and other official functions. Nations may recognize one or more languages as official; the Republic of South Africa designates 11 languages as official, although the most common situation is to recognize only one language in this capacity. A national language symbolically represents the tradition and heartland of the people. A metropolitan language is a language of the Centre states (i.e. First World countries), often an ex-colonial language such as English, French, Portuguese or Spanish. Vernaculars are local languages. All these terms refer to a specific taxonomy of languages. But what, more exactly, is a language?

Blommaert (1999: 431ff) discusses how language is always the product of a politics of representation, involving "massive projections of power, status, values, norms onto the linguistic phenomena at hand" (p. 431). He goes on to present some recurrent features in relation to which the quality of a language is measured, namely structure and order (a product of standardization and institutionalisation); singularity, people, societies and meanings must be monolingual, singular, clear and unambiguous, and languages must express clear and precise thinking; ownership, "a 'good' language is one that is inherited through generations of speakers" (p. 433); expert voices, who produce "legitimising and rationalizing discourses" (ibid.) for specific languages and language forms. All of these phenomena can be encompassed in the notion of a standard language ideology (e.g. Lippi-Green, 1997).

The standard language ideology downplays variation in language, viewing it as deviant and a corruption of a 'pure system'. But variation is in actual fact the very essence of communication. "Variation sends a complex series of messages about societies and the way we position ourselves in the modern world" (Lippi-Green, 1997: 30), allowing the expression of a "web of personal and collective identities".. Part and parcel of the way in which variation is downplayed is through the way structuralist ideas of language as a system — an entity — apart from its speakers is emphasized. This is by far the most problematic aspect of the bulk of linguistic work on multilingual situations, as it cannot accommodate the fact that every speech community is heterogeneous, com-prising different ways of speaking or using a language. Different styles of speech, like different styles of dress, function symbolically to articulate identities and position speakers socially; speakers aspire to use those forms of language that are used by significant others that they might wish to identify with, and utterances come to encode the social position, values and ambitions of the speaker. Language is thus deeply entwined in the social life of speakers and different ways of speaking reflect this. Language is a social phenomenon, par excellens, a form of social action comprising one of the most important ways by means of which individuals and groups construct personal identities, negotiate social relationships, articulate social categories and contest or acquiesce to power relations. In the words of Bambi Schieffelin, "[I]anguage is a system of communicative conventions used to 
establish, maintain and organize social life" (Schieffelin, 1990: 18). The fact that language can be described as a system is a by-product of individuals' linguistic convergence over time in the way in which they use language. When individuals use language they do so with the intention of communicating, referring, complaining etc. They do not consider that they are in any sense constructing language or contributing purposefully to its continuity over time or through space. Speakers just want to get things done with their utterances. However, the unintended consequence of speaking is precisely the creation of language, its continuity and change throughout time (Stubbs, 1995); in the linguistic work that speakers do when they interact, accommodate, and linguistically articulate membership to the social groups to which they (wish to) belong (see Le Page and Tabouret-Keller, 1985), rules are enforced, conventions followed and agreement and understanding between interactants publicly displayed. In this process, conventions of speaking are factored out, and languages are constituted and delimited. This way of conceiving of language, then, suggests that it comes into being through the linguistic work that speakers do when negotiating and constructing social identities. Such a 'fluid' and negotiable concept of language also implies that it is open to systematic conscious and intentional change.

\section{Speech community/linguistic market}

A speech community is usually defined as a social unit made up of people who are in regular linguistic contact with each other, and who either share a language or share conventions for producing and interpreting different language varieties, as well as host-ing shared values and attitudes towards the language(s) and its uses. This definition includes communities where many languages are spoken, but where sufficient consensus among members on the use, interpretation and evaluation of the languages exists.

In fact, speakers shift patterns of discourse and practices of language without necessarily sharing a consensus on what comprises separate languages. Such a vantage point informs a perspective on multilingual societies where speech communities are better seen as a cacophony of intersecting sociolinguistic 'positions of speakers'. According to Hill (1993),

[w]e should assume that speakers confront 'hetereoglossia', which is not necessarily sorted out into a clearly delineated system of codes. Such codes emerge and are reproduced (or not) through what speakers do as they create and deploy a set of interpretative and productive practices that are 'interested', exploiting the available symbolic materials to try to create those conjunctions of forms and meanings that may be most advantageous. (Hill, 1993: 69)

In fact, bilingual societies reproduce themselves through processes of code differentiation and code conceptualisation.

\section{Literacies}

Standard language ideologies have also had consequences for the way in which literacy has been perceived. In recent years, the notion of literacy has been the focus of much debate and contention. Researchers, educators and policy makers have lamented what they consider to be declining standards 
of literacy, developing nations strive to increase their quotient of literate individuals, and civil rights activists underscore how literacy is a prerequisite for true participatory democracy. In most of these contexts, literacy as a notion is treated as non-problematic. It is most often identified with being able to read and write formal, expository, highly edited, content-oriented, decontextualised and non-collaborative/individualized texts. The mastery of this form of literacy is assumed to be necessary for economic development, the development of bureaucratic institutions and institutions of government. On the individual level, it has been claimed to confer psychological advantages, such as the ability to logical thought, on those who master reading and writing. From development discourse we note how

[t]he association of literacy with a modernization syndrome, the conception of mod-ern man, and the development of attitudes and dispositions of flexibility, adaptability, empathy, willingness to accept change, proneness to adopt innovations, all of these were guiding assumptions behind the trend to literacy campaigns in the Third World. (Lankshear, 1987: 42)

The view of literacy as enlightenment and progress and illiteracy as backwardness found in this extract on development is highly reminiscent of traditional discourses on literacy in relation to "primitive" versus "modern" societies. We note here the idea of literacy impacting on society and individuals, and social change resulting from this impact.

This understanding of literacy has been called the autonomous model of literacy by the anthropologist Brian Street
(1984). Street chose this label, because it "rips literacy out of any social context, and treats it as an autonomous, asocial, cognitive skill with little or nothing to do with human relationships" (Gee, 1990: 49). Opposed to the autonomous view of literacy is one that sees reading and writing as embedded in social contexts, constitutive of social structures and linked to discourses of power and identity. Far from be-ing a mere technical skill it is "embedded in socially constructed epistemological principles", and "the ways in which people address reading and writing are themselves rooted in conceptions of knowledge and identity" (Street, unpubl.). This latter concept has been called the ideological model of literacy (Street, 1984), and it emphasizes literacy as a social practice. Literacy is ideological, according to Street because both its meanings and practices emanate from a particular world-view, and from specific cultural practices.

Kulick \& Stroud, (1993) offer an ethnographic account of the processes implicated in a Papua New Guinean villagers' encounters with literacy, and shows how literacy - as we know it - is transformed and (re)defined in the moment it is appropriated by the community. The ways in which these villagers use reading and writing differ markedly from common Western ideas on the uses and meanings of literacy, being used mainly for either religious functions or to encode and articulate specific notions of identity and interpersonal relationships. Villagers' social structures, local institutions and ideologies of Self and Other, fundamentally determine the way in which literacy is adopted in a society.

In multilingual societies, different literacy practices are often associated with different languages. 


\section{First language acquisition}

Standard language ideology has also had implications for how first language acquisition has been viewed. The language used in the first years of schooling has been taken as an implicit yardstick for what children should be acquiring. This is related to the fact that an important part of a standard language ideology is the notion of authoritative entextualisation and something that must be learned from the expenditure of effort through participation in official markets. Standard languages are consecrated varieties. In multilingual contexts, issues of language separation related to the idea of singularity of language and explicitness of thought have dominated the research agendas of applied linguistics. However, in general, the contexts, agencies and institutions where children acquire language are culturally very diverse. Although the norm in research is to assume that the prime linguistic input comes from the mother or father, or some other 'authoritative entextualisation', in point of fact children's language socialization occurs with a range of different caretakers elder siblings, peers at the day care centre, grandparents or other relatives, to mention but a few. In Western communities, children spend increasing amounts of time of time outside the family, and language socialization is distributed among many institutions.

Up until the mid-eighties, studies of first language acquisition paid traditionally little attention to the sociocultural and interactional contexts which frame the process of becoming a socially competent member of the speech community, choosing instead to focus mainly on psycholinguistic and biological correlates of language acquisition. Because of this, many of the routines of managing language socialization found in mainstream families, such as the so called baby-talk register or the important role accorded the language structuring input of parents, so-called scaffolding, which are both socioculturally highly particular to Western contexts, were unreflectingly taken as universal traits of every family. More recently, ethnographically inspired studies, which contextualise language socialization interactions between caregivers and children in socioculturally specific communicative activities, have revealed a diversity of ways through which communities bring up their children to use language. Forerunners in this field such as Ochs (1988), Ochs and Schieffelin (1983) and Kulick (1992), combining an interest in child language acquisition with socialization, have produced important and novel insights into the intimate connections between "socialization through the use of language and socialization to use language" (Schieffelin and Ochs, 1986: 163), and shown how "children and other novices in society acquire tacit knowledge of principles of social order and systems of beliefs (ethnotheories) through exposure to and participation in language mediated interactions" (Ochs 1986: 2). In effect, the structure and content of caregiver-child interactions is "organized by and expressive of wider cultural patterns of thought and interaction" (Kulick 1992: 15), and linked generally to local ideas about language, society and personhood, and to culturally specific conceptions of childhood, learning, knowledge and to roles such as authority and agency. For example, the way that children are encouraged to participate in bed-time stories, through prompts and queries, reflects how American, white-middle class parents conceive of the notion of 
truth (Heath, 1983). Among the Kaluli of Papua New Guinea, a highly common way of addressing children is by means of rhetorical questions, which is one manifestation of the preference among these people for indirect communication (Schieffelin, 1986). Samoan children acquire very early on in their development mastery of semantically complex verbs; this is bound up with the Samoan cultures interactive preferences for affect displays.

L1 acquisition in minority situations is a particular type of process. Minority status for a language often means that it is not used in a full range of functions, as the majority or dominant language tends to out-compete it in public spheres. Even if schooling is offered in the language, the number of years the pupil will encounter the language in school, and the range of functions and type of academic content it is used in are in all likelihood quite limited. All this means that children will encounter the language in a restricted set of styles and with a limited range of role models. Minority languages are also often stigmatised, and both parents and children may internalise the negative attitudes towards the group and its language, minimizing their use of it - at least in public-and preferring to present themselves through the medium of the majority language. Elderly speakers in language communities undergoing shift also tend to be intolerant of what they perceive as younger speakers flawed, incorrect speech. One effect of this is that younger speakers refrain from using the language in the company of elders, which further contributes to undermining it. All of these consequences find a rationale in the standard language ideology. Languages not supported by powerful institutions are rarely considered fullblown languages.

\section{Second language acquisition}

The notion of second language acquisition refers more generally to the acquisition of a language that is not the primary, native, or mother tongue of the learner. Standard language ideologies have also provided the most effective discourses in this field.

According to SLA-theory (see LarsenFreeman \& Long, 1991; Ellis, 1994), a major characteristic of second language acquisition is that it is a successive step-wise developmental progression or approximation to a target language norm. This is either explicitly or implicitly taken to be the standard and accepted educated norm of the community, despite the fact that the primary linguistic input for many minorities is that of the local neighbourhood, which may be far from standard. Learners' attempts to approach the standard throw up so called developmental structures, that is, linguistic structures that do not exist in the target language, but that are thought to transparently reflect the nature of the learners' abilities in it at a specific point in time. In SLA terminology, the stages in the acquisition of a language that a learner produces are known as interlanguages, interim systems of rules and structures that are not yet identical to the target language as it is spoken natively by the target population. An interlanguage is to all intents and purposes the version or model of the target language that a learner has at his/ her disposal at a certain point in his/her acquisitional career.

Learners' interlanguages all share some common, defining features. As they are by nature unstable and transitional, they are linguistically variable and fluctuating. This means that at any point in time, one and the same linguistic structure may be manifested 
differently depending on linguistic context, psychological processing factors etc.

Another important characteristic of learners' interlanguages is that many structures can be described in terms of developmental sequences. Learning a language is not an all or nothing affair. It is a process that takes time, proceeding through a series of steps where any given structure is mastered gradually in acquisition. For some structures, these steps have been shown to exhibit great similarities across speakers and languages, and may also be more or less identical for adult and child second language learners.

Interlanguages are thought to be the result of a variety of acquisitional strategies that learners use; simplification of target language structures and elements, where learners produce scaled down structures with simple phonotactic patterns, reduced morphology and simple unmarked word orders; overgeneralization of target structures where learner's use a correct target language structure, rule or item in a wider range of linguistic contexts than is possible in the target language; transfer, where structures, items and rules from the mother tongue or another language are carried over into utterances in the target language, resulting in interference.

The pattern of acquisition and speed of acquisition are also determined by the type of context in which learning takes place. The contexts in which learners encounter a second language constrain what level of proficiency in it that they will be able to attain. This is because different contexts offer different types of language input, or models of language, for the learner to emulate, as well as provide different conditions for using the language, that is, different opportunities for language output.
What is missing from this account, of course, is the communicative functions that so-called developmental structures might be filling in the local communicative ecologies in which they occur. This is because, from the point of view of standard language ideology, the developmental structures are primarily manifestations of incomplete standard language structures. In one branch of second language acquisition research, the fact that language learning also implies socialisation into the norms of specific primary social groups has given rise to important work about how languages are acquired outside the classroom in informal contexts - outside of standard language ideology (e.g. Rampton, 1995). This type of research takes into consideration the role of language in the social life of speakers and underscores the many ways in which language acquisition proceeds in interrelation with the social uses of language. The view of knowledge, language and learning the individual learner holds, how he/she perceives of the relationship between categories of interactants (child-adult, child-child), how processes of correction and disciplining are accomplished in the society, and how language is used in the negotiation of group or individual identities all deter-mine how language acquisition proceeds. Therefore, socioculturally determined perceptions are important to take into account.

\section{Acquiring language in developing contexts}

The problematic nature of the standard language ideology in relation to language acquisition is particularly evident in situations where new, postcolonial varieties of a metropolitan language are used. A number of social and cultural factors specific to developing nations may 
influence the path of second language acquisition. One such factor is the sociocultural role that a language plays in society. The conceptions and discourses on language learning and use that are associated with the language deter-mines not only what culturally specific elements a learner will use, but also influence the way in which a learner goes about his/ her learning, the social strategies s/he employs and the acquisitional profiles or types of competences that result.

The situation of massive language contact and multilingualism within which learners encounter many ex-colonial languages has a number of implications for language learning. First of all, the fact that the speech community is made up primarily of second language speakers of varying degrees of proficiency means that there is no single stable standard that the majority of the speech community will master. Even though the models of language that children are taught in school may aim at standard varieties of the target language, the model will probably not be significantly represented in the community. In other words, there is little infrastructure outside the school context of any import that might support the acquisition and use of the norm taught in schools. Teachers themselves, often non-native speakers of the ex-colonial language in the majority of cases, may provide inconsistent models of the norm they are sat to teach but do not master. In other words, the main language input that learners have access to is complexly structured non-native varieties. Muysken (1984:101) has pointed out how "L2 learning modelled on the speech production of L2 speakers may be the norm worldwide". The notion of target language in SLA theory has little to say about this situation, based as it is on the conception of the target language as a standard variety spoken by a majority of native speakers in a monolingual speech community (see Long 1993: 206, for further discussion of this point).

Depending upon a host of social factors, different situations of language contact give rise to different types of 'language'. Pidgins and Creoles are contact languages that have arisen in situations of minimal contact between 'target' language speakers and 'learners' who do not share a language. Perhaps the most common case today where minority speakers encounter an ex-colonial language is the school. Platt (1978) uses the term creoloid to refer to second language-based varieties that are largely acquired in formal school settings. He defines this as

[a] speech variety, which has developed through the educational system such that a non-native or introduced prestige speech variety is taught to speakers of another variety (or other speech varieties) in a situation where the introduced variety comes to be used in everyday situations, to be acquired by some or all of the children before they commence school and to become the virtual "native" speech variety of some or all speakers. (Platt, 1978: 55)

Platt (1993) has drawn attention to how the learning conditions of learners who interact daily with a community of second language speakers have some quite specific implications for what type of language is acquired. Referring to the situation in Singapore, he says that

[l]earners of English are continually exposed to SgE [Singaporean English, my note]. At the same time they can practice using $\mathrm{SgE}$ with other speakers of $\mathrm{SgE}$. This constant exposure and use of $\mathrm{SgE}$ has led to a petrified variety. (Platt, 1993: 11) 
The author is introducing here the idea of the importance of output in the reinforcement and consolidation of nonnative norms of language. Fossilization, or petrification, in Platt's idiom, will be the natural result of adapting speech to the non-native social networks that the speaker/learner is part of.

These sorts of situation challenge the assumption that all acquisition or contact with a second language involves speakers in an unstoppable progressive movement towards a 'native' or metropolitan competence in the language. The fact that languages in multilingual contexts show a functional division in use also militates against the idea that all contact should involve complete learning of a target language. Many community members will simply not require the target language for the same range of functions and repertoires as a monolingual speaker of the language, and neither will they encounter this in the language they hear about them. This has implications for the notion of target language entertained in SLA theory, where a monolingual norm is considered to be the endpoint of acquisition. In multilingual contexts, the endpoint of acquisition for the target language must be viewed in relation to the functions of the other languages in the multilingual individual's linguistic repertoire.

Minimally, when studying acquisition, assessment of learners' progress should be made keeping in mind to what uses the language will be put.

Multilingual situations of language contact commonly produce a rich flora of language contact effects in metropolitan languages. Lexical items are transferred from one language to the other, becoming adapted in the process to a new sound system and grammar; clause or sentence patterns as well as pragmatic and discourse notions may calque across language boundaries. As a result of this, there are many stable, recurrent and frequent structures, lexical items and pragmatic processes in ex-colonial languages that can be transparently related to the other languages in the context. As mentioned by Sridhar and Sridhar (1992), some authors do in fact also refer to ex-colonial varieties of metropolitan languages as "interference varieties". Another concept in SLA theory, then, that will benefit from rethinking in developing contexts is transfer. Transfer is, of course, to be expected in multilingual situations, where different languages overlap and complement each other, and where they are used in interaction with other multilinguals who are proficient in the same languages. As Sridhar and Sridhar (1992: 101) claim, "[f]ar from impeding intelligibility, transfer acts as the grease to make the wheels of bilingual communities turn smoothly", serving as "effective simplification, modes of acculturation, [...] and as markers of membership in the community of speakers of a given indigenised variety".. In other words, the lack of transfer between the languages of many multilingual societies would actually impede idiomatic communication and the sharing of cultural presuppositions.

Factors such as the social characteristics, age and gender of the learner are likely to interact in intricate ways in the acquisition of an ex-colonial language. Often variation among learners in this respect is manifested in the form of a lectal or proficiency continuum, similar to post-creole continua such as is found in Jamaica and Guyana. This means that it is frequently possible to distinguish a variety of an ex-colonial language, the so called basilect, comprising features very similar to a creole such as serial verb structures, variable marking of past tense etc. It is also often possible to distinguish a mesolect and an acrolect, 
where the acrolect is the variety closest to the target norm.

Different types of acquisition processes and products correlate with different social conditions and contexts. For example, for $\mathrm{SgE}$, transfer is found distributed mainly among speakers of basilectal varieties, in informal situations and among speakers with low levels of education. However, not all variation in interlanguage systems is due to transfer. Other L2 strategies such as simplification, blends, hypercorrection and analogies also contribute to the variation in different variables. And Fasold has pointed out that a number of features found in non-standard lects in adult speech and that make up variability also occur in the speech of children acquiring the standard language. Chambers (1995) has suggested the idea of "vernacular primitives", linguistic characteristics that are found in many different types of vernacular, creole and child language. When acquiring standard languages that do not permit or include such processes, these "primitives" need to be suppressed.

From the brief account of some concepts of language acquisition given here, we begin to see how specific sociocultural and linguistic contexts may contribute to second language acquisition processes and outcomes. Language acquisition is very much determined by the sociolinguistic heterogeneity and variation in the target language in interaction with the social biographies of the learners. The prevalence of different strategies and the importance of contextual framing factors are relative to the social position of the learner and the possibilities offered by the structure of the community.

\section{Language planning}

The assumptions layered down into the standard language ideology are particularly prominent in discourses and practices of language planning. Christian (1988) defines language planning as

an explicit and systematic effort to resolve (perceived) language problems and achieve related goals through institutionally organized intervention in the use and usage of languages (or language varieties). (Christian, 1988: 197)

A less restricted definition, recognizing the language planning activities of individuals and the wider range of issues that might be planned, is found in Cooper (1989):

Language planning refers to deliberate efforts to influence the behavior of others with respect to the acquisition, structure or functional allocation of their language codes. (Cooper, 1989: 45)

Jernudd and Das Gupta (1971) and Fishman (1973) view language planning as the organized pursuit of solutions to language problems. Fishman (1973) identifies four types of language planning problem, each associated with a particular language planning process: 1) code selection $\sim$ policy formation; 2) management of regional or sociolinguistic variability $\sim$ stabilization and codification; 3) addition of new functions to a code elaboration; and 4) development of functional differentiation between varieties $\sim$ cultivation.

According to Tollefsen (1991), [t]he commonly accepted definition of language planning is that it refers to all conscious efforts to affect the structure or function of language varieties. These efforts may 
involve creation of orthographies, standardization and modernization programmes, or allocation of functions to particular languages within multilingual societies. The commonly accepted definition of language policy is that it is language planning by governments. (Tollefsen, 1991: 16)

Blommaert (1997: 200) also claims that language planning can be understood as a kind of "discourse on language and society, containing ideological assumptions of various kinds". He notes (ibid.: 206) how language planning is actually a theory or discourse on language in its own right, "full of images, preconceptions and assumptions about language, communication and societies, but also about efficiency, rationality and so on". He goes on to say that these assumptions are rarely spelled out, but provide the foundational taken-for-granted commonalities of the field, upon which theorizing and practice may build. Likewise, Tollefsen, in a critical exposé of a classical conception of language planning, which he argues is uncritical and a-historical, proposes an approach where the relationship between the state, language and individual is laid bare, revealing the nature of the ideological interest carried by state intervention in language questions. There is also an increasing amount of research that does in fact lay bare ideological assumptions held by liberal language planning theorists. These assumptions concern the role of language in society and the role of the state in its regulation, as well as deal with the role of language in relation to economic and material development.

A similar concern leads Nesputný (1983) to the conclusion that

[i]t has become obvious today that any theory of language planning must provide a full account of all political values involved in language planning processes [...] This should not be an optional addition but one of the primary objectives of the discipline. I cannot see how language planners working within a rigorous theoretical framework, could be barred from supporting particular values and political aims. All of those I know do take sides. Rather than incapacitating them through calls for sterile neutrality, the theory should assist them in clearly spelling out what their motives and objectives are. (Nesputný, 1983: 3)

Traditionally, language planning has sought to manage multilingualism, reflecting concerns of nation states that there are too many languages spoken in multilingual societies; that there is an enormous range of speakers of different languages (from a mere handful to millions); and that a troublesome vestige of colonialism is the poor correlation between frontiers of speech communities and boundaries of the nation states. Contemporary ideas of development and modernization, conceptions of the role of language in these processes and associated linguistic metaphors of language development tend to converge on the need for one public and regulated language, in the process dismissing multilingual networks to unofficial domains not subject to regulation. Fishman (1994) also finds some grounds for the critique that language planning inhibits or counteracts multiculturalism, although he does not see this as necessarily implying that language planning is inherently "monistic, hegemonic or establishmentarian".

Much of this is formulated by Bamgbose (1994) in terms of the 'the problem of one'. 
In Africa, we are obsessed with the number 'one'. Not only must we have one national language, we must also have a one-party system. The mistaken belief is that in such oneness of language and party, we would achieve sociocultural cohesion and political unity in our multiethnic, multilingual and multicultural societies. (Bamgbose, 1994: 36)

Khubchandani (1974) from an Indian perspective argues that current mainstream activities and philosophies of language planning are not sensitive to the dynamics and nuances of linguistic practice in pluralistic societies. Language planners' attempts to tame diversity and variety in language through the monistic and elite imposition of standard grammars and single styles for each domain of use disregard the flux and multivalence inherent in grassroots multilingualism.

Woolard and Schieffelin (2000) say: The equation of one language/one people, the Western insistence on the authenticity and moral significance of mother tongue, and associated assumptions about the importance of purist language loyalty for the maintenance of minority languages have all been criticized as ideological red herrings, particularly in settings where multilingual-ism is more typical and where a fluid or complex linguistic repertoire is valued. (Woolard and Schieffelin, 2000: 6)

There is a need to recentre "planning theory and practice on the problems of empowering the disadvantaged" (Fishman, 1994: 91). Fishman (1987: 411) sees language planning as the means whereby less fortunate language communities (i.e. those less powerful in their particular confrontation with another ethno-linguistic aggregate) organize their self-defence, as well as their inter-translatability at least to some extent and in some functions vis-à-vis one or another international language. (Fishman, 1987: 411)

Fishman (1994) suggests that language planning should go hand in hand with a necessary reshaping of society in areas associated with language planning in the direction of giving "minorities and subjugated populations a greater opportunity to regulate their own lives and identities" (ibid: 93).

Secondly, he suggests that language planning should be engaged simultaneously with efforts to "foster human freedom, greater power sharing and the ability to resist excessive control over the expression and the selection of ideological and behavioral preferences" (ibid: 94).

Fishman concurs with those who claim that "[1] anguage planning is very frequently part and parcel of [these] two worldwide processes, Westernisation and modernization" and that "[m] uch of language planning is motivated by a desire to render a particular language capable $[. .$.$] of expressing the realia, the$ relationships and the ideas and ideals that stem from the modernized West" (ibid.).

He underscores the necessity for language planners to associate their efforts with questions of language rights, attempt to change the attitudes and understandings of the majority and its institutions, and implement policies in such ways that permits and encourages minorities themselves to regulate their own lives and to share in the policies that affect them.

\section{Corpus/status/acquisition language cultivation}

One prevalent taxonomy of language planning activities distinguishes between corpus, status and acquisition planning (cf. Cooper, 1989). Language planning 
that addresses structural aspects of language is commonly referred to corpus planning. Corpus planning may pertain to any level of language (orthography, pronunciation, syntax, lexicon, pragmatics), and frequently results in a set of accepted 'standard' forms of a language, codified in a normative grammar and dictionary or proscribed rules of usage. Status planning refers to the planning for roles and functions that language(s) are given in society, and the legislation and other means whereby this is accomplished. Acquisition planning finally attends to planning issues in the learning/acquisition of languages.

Shapiro (1989) has said that

[t]he other is located most fundamentally in language, the medium for representing selves and others. Therefore, any move that alters language by centralizing and pruning or decentralizing and diversifying alters the ecology of Self-Other relations and thereby the identities that contain and animate relations of power and authority. (Shapiro, 1989: 28)

\section{Summary}

The standard language ideology that underlies much language planning and policy work rules that linguistic diversity is a problem that demands careful management and containment. The standard language ideology simultaneously provides a perspective on language acquisition and language use that cannot account for the sociosemiotic importance of variation in form and interaction, nor the way in which speakers use language to construct and maintain cultural values and identities. Bilingual programmes are one way in which the assumptions about language acquisition and use implicit in the standard language ideology reach into and determine the day-to-day linguistic realities of minorities. A more grassroots driven approach to language planning and policy work could proceed from the linguistic needs of language minorities, including the promotion and cultivation of alternative literacies in multilingual contexts. In general, the cultivation of diversity should comprise the cornerstone of most work in language planning.

\section{METHODOLOGY OF GURRENT STUDY}

\section{General considerations}

This report looks at what is involved in the failure and/or success of multilingual programmes in developing contexts. The claims that it makes are made of the basis of cross-national comparisons of bilingual education across radically different contexts. More specifically, problems have been addressed in the planning, implementation and evaluation of bilingual programmes that occur in different parts of the developing world, as well as successful solutions or resolutions to these problems. Similar methodologies have been used in other contexts. Kokala (1995), for example, reviews studies by Verspoor (1989) of 21 programmes supported by the World Bank aimed at improving quality of education that were deemed to have been "highly or moderately successful", and by Psacharopoulos (1989) on the failure of educational reform programmes in East African countries. Referring to the critique by Hurst (1991) that studies in pursuit of "recipes for success" neglect the issue of context-boundedness, thereby overestimating replicability, Kokala also notes that studies of "learning from 
failure", of the type "Don't do X", by their very nature can provide little in the way of practical recommendations. $\mathrm{He}$ suggests that

[a] realistic aim for future analysis is to use both the success focused approach and the analysis of failure cases in order to improve our understanding of what appear to be typical, or perhaps necessary, vs. contingent factors in the success of educational reform. (Kokala, 1995: 19)

The presentation and discussion of programmes in this volume culls its data from existing evaluations of bilingual programmes. These evaluations are of different types, in terms of quality, reach and point of departure. Some of them are in the form of academic texts or articles. Other evaluations are unpublished reports of specific programmes ordered by international donors. The bulk of the studies reviewed here are published works. Although this is an advantage as far as quality is concerned, and therefore reliability of results, a more copious breadth of coverage would have been possible had more unpublished materials been available for this study.

Reliance on existing studies means that one has to make do with what one can come up with within a limited period of time. There is a bias in the bulk of the literature on which this study rests towards dealing with questions of bilingual policy formulation and its implementation. Studies that attempt to identify factors affecting the actual operation of the programme and its outcomes through direct observation, for example of classroom processes or community factors, are considerably less frequent. And studies that consider the interactions between different parameters and levels, such as how classroom variables are affected by type of community, are even more rare. One can only speculate on the reasons for this. For obvious reasons, the bias also comes across in the present review of bilingual programmes. Clearly though, it would be desirable to redress this bias in future work.

One very major problem is that there is a lack of information from many countries on the type of provision for multilingual education they make. Where information does exist, important types of data may be lacking from most texts. Few studies, for example, contain information about long term outcomes, such as employment, health status, third generations' educational careers, etc. of participation in bilingual programmes in areas where these have been in force for a number of years. Data on cost-benefit; labour market and further schooling outcomes of students of bilingual programmes would in fact be highly desirable. In other cases, it would appear as though there are strong traditions, or forces of habit, on what type of information is reportable in specific contexts. This is also, of course, a reflex of the particular researcher(s) involved. For example, the data on Latin America comprises some very good studies on classroom interaction, a type of data that is all but lacking in the African context. One reason for this is certainly the involvement of ethnolinguistically trained American researchers with specific interests in this area.

One implication of the somewhat arbitrary character of what information is available is that grand generalizations and quantitative conclusions are difficult to make. Another implication is that the present overview can make no pretensions to being exhaustive, or of covering every parameter for every context. The type of information available makes a selection of studies 
and a selection of problems a more appropriate approach, and the design of the report is in the form of "illustrative case studies". If a programme has been considered to be of interest in some respect which addresses the main point of this review, namely to point to factors contributing to success or failure, it has been taken into consideration.

Different evaluations of the same types of bilingual programmes may occasional-ly generate contradictory research results. One reason for this is that programmes are built up of a number of component parts that each on its own might not be applicable to particular contexts. Yet another, and related reason for the contradictory results is that the programme may have been evaluated for outcomes for which it was not designed. As the initiative to evaluate a programme may come from many different sources with quite different agendas (parents, professional educators, educational administrators, funding agencies, or other government bureaucracies), the questions asked of the programmes may differ. A complicating factor in analysing programmes is that it is sometimes difficult to know to what extent the bilingual curriculum is implemented, if it exists, due to e.g. classroom conditions. There are numerous cases in the literature where programmes have been shown to have had adverse consequences due simply to the fact that the individual teacher or school had decided not to follow policy decisions. To judge a programme negatively on the basis of such a situation is hardly fair.

Furthermore, contradictory research and evaluation results may arise because data is interpreted without recourse to a theory, that is, atheoretically, so that interpretations are not constrained by common frame of reference. As contexts, pupil characteristics and teacher attributes change, so may the success of any particular programme. A study evaluating a particular project may convincingly demonstrate the efficiency of the project's approach to solving a particular set of problems, but it is difficult to transfer that experience to a different setting unless we understand why the approach does or does not work for a particular context. The problem that is high-lighted here is that of generalizing a specific design of bilingual programmes from one context to another. To understand why an approach works for a particular set of children in specific local context, we need an interpretative framework that will aid us in understanding the values and meanings of the parameters. This is also what Cummins has in mind with the following quote:

Data or facts from bilingual programs (or any other programs) only become interpret-able for policy purposes within the context of a coherent theory. It is the theory rather than the individual research findings that permits the generation of predictions about program outcomes under different conditions. Research findings themselves cannot be directly applied across contexts. For example, the fact that kindergarten and grade 1 Panjabi-background students in a Panjabi-English bilingual program in Bradford, England, learned English just as successfully as a control group in a traditional English only program, tells us very little about what might happen in the case of Latino/ Latina students in the United States. (Cummins, 1996)

A common type of problem that evaluations in third world contexts has to grapple with is that the programmes 
often take place with a paucity of resources. On the one hand, bilingual education, as any education, can hardly be expected to succeed if sufficient materials, teacher training, instructional time etc. are not made available. On the other hand, those evaluations that show positive effects for bilingual education do so under extremely advantageous conditions compared to mainstream education. For Africa, more specifically Nigeria, Akinnaso (1993) reports on an evaluation of the Ife project that shows evidence of bilingual advantages in a range of areas. This was a project initiated by the state of Ife that involved students being taught in the mother tongue throughout the full six years of primary schooling. A comparative study be-tween the Ife project and Nigerian public schools, where mother tongues are used only in the first 3 years of schooling showed that (i) students in Ife performed better cognitively and academically, (ii) mother tongue (Yoruba) educated students were just as proficient in English as those students who had been educated in English in the mainstream programme, grades 3-6 (iii) cultural, affective and sociopsychological gains were made by students educated in the mother tongues. Akinnaso, however, does point to some problematic points that make a straightforward and categorical interpretation in favour of 6 years mother tongue education difficult. For example,

the results were compounded by a combination of several non-linguistic factors, including curricular changes; the use of new course materials; the use of experienced teachers for whom additional training was also provided; changes in classroom practices; and greater attention than usual (especially in English Education) to experimental classes. (Akinnaso, 1993: 70)
All in all, evaluation studies are therefore highly problematic sources of data on which to base generalizations. This implies that the most qualified aspect of this report is in the critical reviewing and weighing of different studies and their findings. This process essentially involves familiar techniques and discourses of academic peer commentary. Therefore, where appropriate, single case studies are provided with a metacommentary that may assist the reader in judging the efficacy of studies and their conclusions.

\section{Types of bilingual programme}

I noted in the previous section that the claims made in this text emanate from a literature review of a range of bilingual programmes. However, the concept of bilingual programme is a rather elusive one. Hornberger (1991) points out that much of the literature around bilingual models suffers from conceptual unclarity on four counts; (i) the same label may be used in different ways in different typological frameworks, (ii) many different labels are sometimes used for basically the same model, and (iii) the epithet bilingual education is applied to models which strictly speaking do not use the mother tongues of their educational constituencies, that is models that all employ some form of monolingual teaching, although in multilingual contexts. Finally, (iv) there is a confusion between models that are defined in terms of their goals, and models that are defined in terms of their construction or the contexts in which they occur.

Examples of (i), where the same label is used to refer to different models abound in the literature. Maintenance models may, for example, refer to programmes that emphasize only the preservation of oral skills in a language, or to programmes that also attempt to develop literacy 
skills and cultural competence in two languages. This programme model may at times be distinguished from enrichment models and on other occasions be subsumed within them (Crawford, 1989).

The casewhere differentlabels are used for the same model, (ii), can be illustrated with the label transitional programme, which is also called assimilationalist or compensatory (Crawford, 1979). Maintenance bilingual education may in some literature be found under the rubric developmental bilingual education or language shelter.

Case (iii) where programmes are called bilingual although technically they only use one (often majority) language with bilingual pupils come under different labels; replacement, segregation, submersion and mainstream, structured immersion, monolingual education, English as a Second Language. It should be noted that the term bilingual is used mainly in a popular and/or political sense, as they are from a technical point of view clearly not bilingual. The distinction between popular and technical conceptions of bilingual education was proposed by Cazden and Snow (1990).

Most interestingly, however, is Hornberger's claim that the majority of typologies do not distinguish between programme goals and objectives, the structure of the programme and contextual factors. As one example of this, she mentions that maintenance programmes are sometimes "defined in terms of its goals, in this case the maintenance of the ethnic language and culture (e.g. Fishman, 1982) and sometimes in terms of its programme structure; the curricular maintenance of the ethnic language as a medium of instruction throughout the years of schooling (Mackey, 1972)". However, as Hornberger points out, if there is no relevant programme structure in place, the goal or intention to maintain the ethnic language will hardly be implemented. Likewise, if the language is not used outside the school environment, a maintenance programme structure might not succeed in fostering language maintenance.

For the purposes of the present report, the following labels for types of bilingual programme will be used. Where these labels are not appropriate, care has been taken to determine and report on the exact characteristics of bilingual education option.

\section{Transitional programmes}

Typical transitional programmes have the goal of assimilation and social incorporation of language minorities in the national society (Hornberger, 1991) through language shift. The rationales for this model are various, but all touch upon the need for the minority language speaking child to function in the majority language community as quickly as possible; equality of opportunity with mainstream peers and maximization of minority pupil performance are the main priorities. Linguistically, the aim of this model is monolingualism. Behind the rhetoric of equal rights and opportunities, however, we may glimpse the mainstream societies concern with ghettoisation, and the desire for integration/assimilation of minorities to mainstream values and behaviours.

\section{Maintenance programmes}

A typical maintenance programme aims at a set of pluralistic goals, for example, strengthened cultural identity and civil rights affirmation, through the sheltered maintenance of the mother tongue. A distinction in maintenance goals has been made between static maintenance, referring to the goal of keeping children's language skills at the level they were at when entering school and preventing language loss, 
and developmental maintenance, which aims to develop the students' skills in the minority language to full proficiency (cf. below on enrichment programmes). One reason cited by Baker (1996) for giving a maintenance programme is that a minority language is easily lost, but a majority language is easily gained.

\section{Enrichment programmes}

Enrichment programmes aim toward both maintenance and the development and extension of the minority language, the affirmation of cultural pluralism and an integrated national society based on autonomy of cultural groups (see Fishman, 1976 and Hornberger, 1991).

\section{Restorative programmes}

These are programmes that aim to revitalize a language and its community of speakers. Adults partially competent in the language are also targets for this type of programme.

\section{MULTILINGUALISM IN DIFFERENT SGENARIOS OF LANGUAGE CONTACT}

\section{Introduction}

Speakers of non-metropolitan minority languages have found themselves historically in various situations of subordination vis-à-vis metropolitan languages, such as English, French, Portuguese or Spanish. In more modern times, other languages of wider circulation have also come to exert increasing pressure on language minority communities such as Swahili in East Africa, or Creole in Guinea Bissau. In this section, I shall explore briefly some typical linguistic ecologies found during the colonial and postcolonial eras and address some of the implications that these different ecologies have had on speakers' use of language and patterns of multilingualism. The chapter will conclude by sketching some possible imminent and future multilingual scenarios in the wake of post-industrial globalisation.

\section{Multilingualism in colonialism and postcolonialism}

During colonial times, the regulation and control of languages on behalf of colonial powers was an important instrument of colonial management. Colonial language policies ensured that all important public societal functions were mainly conducted in the metropolitan languages alone, and that local languages were used more or less exclusively in private spheres. Accompanying this division in use was an ideological stance towards local languages as chaotic, imprecise, lacking in abstractness and not at all 'real' languages, just 'dialects'. In this way, language policies helped to reinforce the division of material and economic power between colonial master and colonized, and controlled access to scarce resources. One example, taken from preindependence Mozambique, will suffice to illustrate the ways in which language was used in this way to monitor, control and demographically divide populations.

In colonial Mozambique, Portuguese had a legally stipulated role as the sole language of public life, while indigenous languages were confined to use in private domains. This language policy primarily served to bolster a labour legislation that regulated the definition, organization and control of the work force in favour of the supremacy of white Portuguese labour. The policy also effectively regulated which social spaces blacks and whites could inhabit, and the nature of the different identities and privileges 
they could aspire to. The notion of assimilado ${ }^{1}$ was important in this context. Assimilados were allowed to remain within city limits after nightfall, live in white residential areas and use public utilities such as cinemas and restaurants that were otherwise reserved for whites (Marshall, 1993). An assimilado, in other words, was a kind of honorary white, and one condition blacks had to meet to attain assimilado status was to master Portuguese.

More generally, in the postcolonial era, a time of upheaval and change, the status and functions allocated to different languages in multilingual polities were determined primarily by attempts to balance demands for national unity with recognition and affirmation of diversity. This tension generated language ideological debates and policies of very different natures (see Edwards, 1993: 25):

1. elevation of a strong internal variety to national/official status (Somali)

2. promotion of a supra-ethnic variety with neutral connotations, but which is still indigenous to some degree (Swahili)

3. promotion of a standardized version of a widely spoken but dialectally fragment-ed indigenous vernacular (Standard Malay in Brunei)

4. continuation of a colonial language of wider communication (LWC), sometimes in indigenous, local or nativised varieties

5. some combination of (i) - (iv).

Again taking an example from Mozambique at independence, questions of language were intimately tied to the development and consolidation of the new postcolonial nation state. Portuguese was made the official and working language of the state, and ideologically portrayed as a national heritage at the same time as it was taken to represent modernity, order, consensus and a unified nation state. National languages, on the other hand, were associated with tradition, ethnic division, and colonial control. Although they were said to be essential for a community's access to its historical roots, and for preserving traditions and customs, they were given no official formal recognition in modern sectors of Mozambican society. In fact, in the early days of independence, national languages were more or less prohibited for use in formal contexts. One effect of this was that local people were effectively barred from having a say in the running of their own lives - unless they had a knowledge of Portuguese. Ultimately, this linguistic marginalisation contributed to their poverty and powerlessness.

At the same time that the governing party FRELIMO was ideologically refashioning Portuguese as an integral part of the invention of modern Mozambican society, it was also staking claims to the language. Portuguese was hailed as the language of the FRELIMO party. What this in fact did was to effectively link the people to the party through every public act of interpersonal communication in which Portuguese was used. It created a 'nation-state habitus', and provided FRELIMO with an important strategy in its efforts to gain political legitimacy (cf. Stroud, 1999).

1 An assimilado was a black Mozambican who had been given the privilege of learning Portuguese and taking a Portuguese name. To become an assimilado, the black Mozambican had to speak Portuguese, believe in one (right) god, be monogamous and employed. 


\section{Global and the local economies}

Today's post-industrial global economy, an economy "in which capital, production, management, markets, labour, information and technology are organized across national boundaries" (Castells, 1996: 18), is radically transforming the linguistic economies of many postcolonial nations in as yet uncharted ways. On the one hand, these processes are reinforcing the de-emphasis of local and regional institutions, ways of life and languages that have been typical of prior colonial and postcolonial developments. One example of this is from Tanzania, where the World Bank and the International Monetary Fund, called in to help halt the country's recession, were able to exert considerable leverage on policy makers to force a stronger emphasis on English. English has also become more important vis-à-vis Swahili with the rise of a parliamentary and representative democracy in Tanzania (cf. Brock-Utne, 2000).

On the other hand, the development of global markets have led in some cases to a strengthening of local indigenous languages vis-à-vis the official nationstate language.

Speakers of other metropolitan languages, such as Portuguese and French particularly, cannot have avoided feeling the (socio)linguistic impact of English on their languages through globalisation. In French and Portuguese speaking Africa, the growth of multiparty politics and the opening up of trade networks with English speaking neighbours (especially with South Africa since the removal of its apartheid government) has meant that French and Portuguese have lost some of their importance and prestige on local national arenas. If we are right is assuming that the relative degree of subordination of a minority/indigenous language is in direct proportion to the perceived importance of the metropolitan language, then the weaker this language becomes, the relatively more stronger local languages become (cf. Grin, 1996).

Another aspect of globalisation with potentially positive - or at the very least ambivalent - consequences for minority languages is the development of the knowledge-economy. The accumulation of knowledge and information is crucial to product design, production and innovation, and Castells' claims that "the level of efficiency in acquiring, processing and deploying knowledge now constitute the strategic factors in both competitiveness and productivity for firms, regions and countries" (Castells, 1989: 19). A knowledge-economy may hold far-reaching implications for minority languages and their speakers. This type of economy requires "new flexible work practices organized around co-operative team work and skills transfer ability or multi-skilling - a "flexible specialization" (Rassool, 1999: 332) as well as the horizontal dispersal of control ('new work orders' (cf. Gee, 1990, 2000)). Technological processes need to be integrated into the entire social process. Production and consumption of knowledge - need a highly skilled workforce. According to Rassool (1999: 137), "the full value of the development of technological and scientific capabilities lies in the extent to which they can be embedded in existing structures and blend in with pre-vailing traditions and cultures" (see also Delors, 1996). At least on paper, a 'democratic' knowledge economy should favour the use and development of local languages.

Post-industrialism also has a range of potential implications for the teaching of indigenous languages. In 
an information based economy, higher order literacy and communication skills are seen as central for growth (Rassool, 1999: 134), as information sharing, negotiation, consultation and cooperative teamwork require a range of sophisticated communication and literacy skills. These skills frequently involve the formation of new codes, among them multimedia 'literacies'. In other words, new demands are placed on the linguistic skills of workers. The language skills and abilities that speakers of indigenous languages need to cultivate are those that permit 'horizontal' creative and problemsolving forms of communication (forms of language modernization that permit the expression of a "culture of divergence"); that can integrate ideas and products into existing social networks (contextualisation); that encourage diversity (hybridity); and that focus on multimodality. The types of language and literacy functions that speakers need to cultivate in their languages should centre on developing interpersonal knowledge networks, as well as identity constructions (see below under co-operative methodologies). In other words, teaching in and through local indigenous languages need to centre on difference, change and creativity.

The impact of the global economy on language dynamics and patterns of bilingualism is mediated by the types of networks or life forms that individuals and groups have access to. Factors such as individuals' gender, neighbourhood, profession, and education determine what networks and identities are available to any one single person at any one point in time. Depending upon where community members are located in the urban power matrix, their interactive networks will differ. In the words of Gupta and Ferguson (1992), [p]hysical location and physical territory, for so long the only grid on which cultural difference could be mapped, need to be replaced by multiple grids that enable us to see that connection and contiguity more generally, the representation of territory - vary considerably by factors such as class, gender, race and sexuality, and are differentially available to those in different locations in the field of power. (Gupta and Ferguson, 1992: 19-20)

This in turn determines what type of language markets an individual will have access to and what type of multilingual practices s/he will use on a daily basis. This is one important determinant of what Warman (1980: 304, quoted in Hill, 1985: 735) refers to as the "symbolic flow" — the "words and ideas [which] actually connect the modes of production and shape their relations toward the inside and the outside".

There is a great deal of work that tries to explain local structures of multilingualism with reference to superordinate political and social structures at the regional and global levels (Hill \& Hill, 1986; Hill, 1993). For example, Jane Hill (e.g. 1993) applies a Bakhtinian conception of language as heteroglossic to the analysis of codeswitching and other contact phenomena occurring in the speech of Spanish Mexican speakers. These speakers find themselves participating in both a traditional local economy, characterized by reciprocal relations, and in the peripheral margins of an industrialized and global economy. Hill explores how the juxtaposition of two languages can be understood to be the speakers' presentation of two "voices", that is, ways of speaking that foreground and construct specific interested positions and identities. 
Another researcher, Susan Gal (1989), applies such a perspective to the speech of immigrant youth in Germany, treating codeswitching as "speakers' symbolic responses to the differing political economic positions in the long standing system of core periphery relations" (p. 357).

\section{Summary}

In this section, I have wanted to present a view of multilingualism as a set of language and cultural practices for negotiating, defining, constituting, (re)affirming and symbolically representing the social identities of individuals and political relationships of speech communities in contact. Linguistic outcomes of contact may comprise the degree of access speakers have to different languages, the functions that are allocated to these languages, as well as the symbolic value accorded to them. In following chapters, we will see that a range of social, historical, political and ideological factors determines how power relationships between communities in contact become trans-posed into patterns of multilingualism.

\section{LANGUAGE LOSS AND SHIFT}

\section{Introduction}

One implication of contact between speech communities or languages of unequal power is that speakers of a weaker language tend to shift more and more into using the more powerful language in extended functions and domains previously reserved for the minority language. Over time, this may lead to the loss of the minority language as a viable means of communication and social cohesion for the group.
Clearly, these sorts of dynamics have numerable implications for bilingual education programmes. How to account for language shift and loss? What are its implications?

\section{Accounting for shift and loss}

In the preceding chapter, we have noted a plethora of sociological, political and economic developments accompanying various historical phases from colonialism to globalisation. Some authors have suggested that the detailed specification of crucial macrosociological factors such as industrialization, migration, colonial domination, urbanization, etc. and their combination might help account for under what conditions language shift/loss will occur. Others authors such as Gal (1979) and Kulick (1992), however, have drawn attention to the fact that societal institutions and global developments in themselves are hard put to account for the linguistic behaviours found in a speech community. Gal writes: "What is of interest to know is not whether industrialization, for instance, is correlated with language shift, but rather: By what intervening processes does industrialization, or any other social change, affect changes in the uses to which speakers put their languages in everyday interactions?" (Gal, 1979: 3). And Kulick says: "To evoke macrosociological changes as a "cause" of shift is to leave out the step of explaining how such change has come to be interpreted in such a way that dramatically affects everyday language use in a community" (Kulick, 1992: 9). Studies that have followed this focus on speakers cultural conceptions of language, their language ideologies, as well as the symbolic and social messages carried by different languages, and their 
role in the articulation of complex social and individual identities, have provided sophisticated narratives of language loss and shift (Gal, 1982; Hill and Hill, 1986; Kulick, 1992). For example, Mertz (1989) points out that her Gaelic informants stopped speaking Gaelic to their children because they thought it otherwise would interfere with their children's acquisition of English. And Kulick (1992) argues that Gapun villagers' shift to Tok Pisin from Taiap can be under-stood with reference to how highly salient and desirable traditional aspects of Self have become encoded in Tok Pisin over the years.

\section{Implications of shift and loss}

A situation of contact between powerful and less powerful languages results in various types of structural, stylistic and lexical changes - predominantly found in the subordinate language. Some of the changes are an effect of interference from or convergence with the dominant language, where lexico-syntax may be adopted wholesale into the subordinate language. Other changes, including stylistic shrinkage, come about from the restriction in minority language use that accompanies its limited functional use in society in comparison with the dominant language. When these types of developments occur, they invariably have an impact on speakers' perception of how appropriate the minority language is as an instructional medium at school. It is also important to be aware of the fact that the same processes that produce language shift and concomitant loss of stylistic and structural options may produce innovation and creativity in the minority language (cf. Gal, 1996).

\section{DISCOURSES ON MULTILINGUALISM}

\section{Introduction}

The notion of linguistic ideology is crucial in accounting for the mechanisms and institutions that reproduce specific types of multilingual practice. The linguistic ideologies that speakers entertain provide the mediating link between social events, structures and relationships, on the one hand, and actual language practices on the other. Ideologies of language provide speakers with discourses that circumscribe the meanings of bilingualism and define the nature of 'being bilingual' for individuals. These regulatory discourses are the outcome of discursive struggle and contestation in language ideological de-bates. Blommaert notes that "debates are more or less historically locatable historical periods in which a struggle for authoritative entextualisation takes place" (Blommaert, 1999: 9). They involve a struggle over definitions or representations of reality; "various representations of reality are pitted against each other - discursively — with the aim of gaining authority for one particular representation" (ibid).

The ways in which such discourses work to define the Other can be seen in some of the earliest discourse on bilingualism that were produced in conjunction with the turn of 20th century USA. The waves of southern European immigrants that came to North American shores in search of work and refuge provided an unwelcome source of worry for official gatekeepers, as they were considered to be of an inferior racial quality to the earlier Northern European immigrants. Discourses on the disadvantages of bilingualism interlocked with these concerns. More contemporary 
thinking on bilingualism has lifted forth other themes. For example, during the seventies and eighties discourses on bilingualism were most often phrased in conjunction with semilingualism. Interestingly enough, it was typically welfare states, such as Australia, Northern Scandinavia, and Canada, where the notion of semilingualism gained its strongest foothold. Today, most discourses on bilingualism are framed in tropes of 'contagion', expressing, for example, parents' fears that non-native language practices will spread to their mother tongue speaking children. Stroud and Wingstedt (1989) have pointed out how the notions of 'semilingualism' and 'Rinkeby Swedish' function as metaphors for inter-group relationships, and that the changing message carried by the different metaphors is a barometer of society members views on immigration and non-nativeness more generally. More wide-spread discourses bearing a similarity to semilingualism portray multilingualism as giving rise to cognitive confusion in the individual through the competition of two unequal systems. Economic discourses on multilingual education are pervasive and influential.

All these discourses are naturally cited by critiques of bilingual education. On the societal level, multilingualism, involving as it does many languages, is said to be technically unmanageable, and a major contributing factor in retarding national development. In the neo-liberal discourses of globalisation, however, multilingualism is being promoted as "productive diversity" following the recognition that each specific language community is a potential market for a niche product tailored to their needs and sold in their language. These discourses are global entextualisations, in the sense that, even though they address domestic policies and concerns, they are international and downloadable.

North and South relationships have generated a host of conceptions of multilingualism and local languages. Fabian notes how these conceptions fit into a rhetoric of cultural dominance, providing the ideological arguments that colonial powers needed to legitimise their colonial rule (Fabian, 1986). Even today, the discourses continue to metaphorically define relationships between Modern Self and Postcolonial Other (cf. semilingualism and Rinkeby Swedish). This section will deal with some of the common ways of talking about multilingualism, catastrophic or doomsday discourses on multilingualism, what we could call myths of multilingualism. Along the way, attempts will be made to offer some more balanced commentary on the claims made by these discourses.

\section{Multilingualism in society}

Multilingualism is frequently represented as something that interferes with the smooth running of a society. The assumption seems to be that if a society hosts many languages, this will automatically create misunderstanding and cross-communication, and at the very least require the provision of costly translation and interpretation services to cope. However, this representation rests on a misconception of the true nature and extent of linguistic diversity in multilingual societies, as well as misunderstanding the role played by linguistic diversity (see also above on 'standard language ideology').

Firstly, we cannot simply equate language differences and linguistic diversity with communicative breakdown. Djité (1993: 150) has noted how "purely linguistic demarcation of languages or dialects...does not translate into actual boundaries of communication". Speakers 
who, to all intents and purposes, speak different languages often do manage to communicate in practice. Xhosa speakers from the Western Cape in South Africa can understand speakers of the Changana language from Southern Mozambique. These in turn can communicate with speakers of SiSwati, the official language of Swaziland, and even with speakers of Shona spoken in Zimbabwe and Mozambique. One way in which this is made possible is through speakers capitalizing on existing formal similarities between languages, as well as learning on line to recognize equivalent forms of the same structural items - just as a Dane and a Swede, after an initial period of non-understanding, may start to listen in and begin to discover the correspondences that exist between their two languages. Another way communication can be facilitated is to go via a third language. In the African context, a rudimentary knowledge of Zulu can be employed as a template on which to model moment-to-moment linguistic adaptation, thereby building a 'bridge' between the Changana, SiSwati and Khosa. This means that millions of people, rather than being divided by their multilingualism in African languages, are actually linked into a regional speech community through these languages. The tendency to take for granted that formal differences in language equal communication difficulties in practice emanates from linguists' focus on language as a system rather than as a set of negotiable communicative practices. Hymes (1984: 8) writes "One must ask, not about genetic relationships among languages and objective linguistic demarcation of dialects, but about communication relationships among persons and groups".

Another facet of this particular myth of social disorder following from multilingualism addresses urban areas. Urban areas in developing nations comprise a chaotic mix of different generations speaking widely different mother tongues and interacting socially in a host of ways in complex demographies of contact. This is said to precipitate a low level of linguistic intelligibility, and make choice of any bilingual education well-nigh impossible. It is true that one of the most massive demographic changes in recent years in developing countries has been the growth of cities. Gilbert and Gugler (1992) note that

[f]uelled by changes in the countryside, high rates of fertility, falling death rates and rapid cityward migration, most Third World countries have been transformed from rural to urban societies in two or three decades. The larger cities have been expanding rapidly, often doubling in size every fifteen years. (Gilbert and Gugler, 1992: 7)

Once again, cities are far more linguistically homogenous that they would appear to be at first blush. The constellation of contact factors in the urban multilingual ecology provides a fertile nest for the evolution of contact varieties of languages, such as the Sheng spoken in Nairobi. In the Central African Republic, creolised Sango and French coexist among the urban population; in Zambia, Lingala and French, in Kinshasa, Town Bemba and English are spoken by urbanites, and Swahili and English are used in the cities of Kenya.

I noted previously that one factor contributing to tropes of linguistic disorder and chaos is the tendency for linguists to want to multiply the number of languages in a territory. As a consequence, descriptions of multilingual societies frequently sound like veritable Towers of Babel: Papua 
New Guinea, for example, is said to host 850 languages (an estimated $15 \%$ of the worlds languages spoken by a mere 4.5 million people); the African continent is said to be able to boast approximately 1000, with Nigeria alone accounting for as many as 400 languages (cf. Krauss, 1992). What these types of account ignore, however, is that languages do not just exist, they are 'written' or brought into existence - they are constituted; where one account defines two separate languages, another might only see two varieties of the same language. And, following from this, different ways of describing languages will provide very different pictures of the linguistic diversity of a nation. In Mozambique, for example, linguists have been debating a while what comprises the best representation of the language situation. A large amount of linguistic evidence has been offered for the existence of approximately 24 languages. At The First Conference on Mozambican Languages, held in 1988, even more language labels were conjured up, as varieties that researchers on the basis of lexical and structural evidence had considered to be forms of the same language, were in fact claimed to be separate languages by their speakers. On the other hand, influential Mozambican linguists, following the linguist Guthrie's criteria, suggest that the majority of Mozambican languages fall into four main language groups. Within these language groups, most varieties, at least those centrally placed, are considered to be mutually intelligible.

So far, we have seen that structural diversity across varieties does not necessarily mean that speakers of these languages will not be able to understand each other, and that, beside this, the extent of structural diversity among languages is probably exaggerated in some cases. However, not only is the extent of language diversity and its consequences misperceived, but the role played by multilingualism in society has also been subject to misinterpretation. Firstly, rather than comprising a hurdle to overcome, a strong case can be made for viewing multilingualism as a societal resource; in multilingual contexts, people rely on different but interacting language repertoires to organize and manage social life at the level of the region or locality in which they live and work.

Secondly, the use of different languages allows speakers to contextualise and frame meanings in alternative ways, thereby providing fresh perspectives on events. It has long been known from bilingualism research that switching languages in a communicative interaction carries a host of stylistic and rhetorical, interactional and discoursal meanings. Multiple languages are resources because they allow the simultaneous expression of many layers of meaning. Code-switches serve a number of important message and contextualising functions and permit complex expressions of meaning.

Thirdly, the more languages involved in any communicative event, the more people of diverse backgrounds can participate in these event, thereby pooling multiple voices. The recent multiparty elections in Mozambique provide an interesting case in point of how use of vernacular languages, far from jeopardizing the flow of information, was actually essential to the success of the electoral process. Formulating political messages in local languages and Portuguese facilitated the electorates under-standing of how political views would work for their local context, as well as allowing insights into how local affairs would impact on national issues. (Liphola, 1998). 


\section{Multilingualism and national economy}

A pervasive myth about multilingualism is that many languages and linguistic heterogeneity in a nation is associated with low per capita GNP. In evidence of this claim, proponents point to the relatively higher levels of poverty in highly multilingual parts of the world such as India and the continent of Africa. Fishman (1991a) provides a critical analysis of this claim. Using advanced statistical techniques, he correlated 238 different economic, political, social, cultural, historical, geographic and demographic variables from across 170 countries to GNP, only to find that linguistic heterogeneity bore no predictive value for the level of per capita GNP (Fishman, 1991: 13). And, in fact, Fishman and Solano (1989) even suggest that the existence of lingua francas and bilingualism enable many polities to attain a higher per capita GNP.

Another author who has addressed this issue is Gonzales (1993). He explored the relationship of language, especially multilingualism, to the level of national development. National development was assessed on the basis of a large variety of variables comprising per capita income, the balance of wealth distribution, citizens' perceived security and wellbeing, the level of protection of legal and other rights (including linguistic rights) that the state offered, the extent to which law and order prevailed, level and quality of education, quality of environment, the availability of housing, and whether the community had an adequate supply of food and other necessities (Gonzales, 1993). Gonzalez correlated this measure of overall socio-economic development of the country with a language welfare index. The main finding was that although there was a correlation between literacy and socio-economic development, there was no significant correlation between state of national language and socioeconomic development.

The other side of the coin is the claim that developed nations are more linguistically homogenous. However, Lieberson (1980: 12) has pointed out that "essentially no causal linkage exists in either direction between changes in a nation's development and its level of linguistic homogeneity".

Irrespective of what beliefs we entertain with respect to multilingualism, linguistic heterogeneity and development, most of us would agree with the argument that nation states need a metropolitan language of wide currency, such as English. The argument goes that metropolitan languages are the way to full global participation, as only these languages can provide the necessary access to technology and scientific knowledge. Even this claim is subject to criticism, however. While acknowledging that a nation-state needs recourse to a language of wider communication in today's global economies, this does not imply that every citizen needs to master a metropolitan language (as a first language). And, at the level of anecdote, critics often single out Japan as an example of a leading economy that uses a language other than English, French, German or Portuguese.

Contrary to those supporters of modernization theory who see the use of metropolitan languages as the sine qua non of progress and prosperity, Djité (1993) and Robinson (1993), among many others, have argued that economic and social development in a society may actually be enhanced through employing indigenous local languages.

This is because "the actors of change remain the people themselves" (Djité, 1993: 150); employing languages that 
the grassroots themselves master will facilitate the transference of technology and know-how to the people who need them most. Contextualising knowledge development in local languages will allow for greater participation among more people, and should also permit an easier integration and adaptation of new developments to local social institutions and decision structures.

In fact, a case could be made that reliance on metropolitan languages in developing contexts has "significantly contributed to the socio-economic and political instability of most [African] countries" (Mazrui, 1997: 44). Mazrui notes how the process of spreading metropolitan languages, today spearheaded by institutions such as the World Bank and IMF, is part of a "wider economic agenda intended to meet the labour requirements of foreign capital" (ibid.) by helping in the "creation and reproduction of [this] labour hierarchy" (ibid.). He claims that "[i]n essence, the World Bank's proposed educational configuration in Africa demonstrates the continued role of instruction in Euro-languages in creating and maintaining an economy dominated primarily by foreign economic interests and, secondarily, by a small aspiring African bourgeoisie" (ibid.). In Mazrui's conception, the (uncontrolled) spread of metropolitan languages is a mechanism to ensure the production of cheap labour for the rich post-industrial giants. On a national level, the spread of metropolitan languages also helps foster and consolidate a socio-political structure that serves the interests of the local and regional elites. Because world markets are also indigenous markets with local elites tightly integrated and supportive and reproductive of the values that dominate these markets, metropolitan languages come to constitute an important form of symbolic and valued capital with which these elites can vie for a spot on this global economic arena. In other words, the collusion of the local elites reinforce tendencies towards exclusive use of metropolitan languages on national markets.

Another reason for employing indigenous languages is that these languages do actually comprise an economic asset in their own right. In many African states there is a large informal economy that may in some estimates comprise up to $70 \%$ of a country's GNP. Most of the economic transactions through which these economies function are conducted in local languages not metropolitan languages. Recent years' globalisation has brought about the growth and diversification of niche markets and niche products that address the needs and tastes of select consumers. These products need to be sold, which means that markets must be created and reinforced through advertising and information. In this context, knowledge of different modes of discourse and discourse strategies are vital, as is access to a diversity of languages, dialects and discourse registers. Multilingualism is fast becoming an economic necessity rather than a liability. In the words of Michael Clyne, this development involves a shift from a socially motivated language maintenance to a focus on an economically motivated language learning, and a rhetorical emphasis on 'productive diversity' (Clyne, 1997).

While we can safely say at the level of society that multilingualism does not have any negative impacts, at the level of the individual, ignoring minority languages may have a range of adverse consequences. Kaplan and Baldauf have itemized a number of social costs that accompany linguistic disempowerment of minority groups. Because minority groups tend to have unequal access to 
employment, they consume a larger share of welfare resources and live in substandard housing. Unequal access to health care in minority groups can be correlated with higher infant mortality rates, higher birth rates, more communicable diseases, higher incidence of accidents, and shorter life spans. Minorities also tend to have higher rates of criminality and to do poorly in the education system (Kaplan and Baldauf, 1996: 161).

\section{Multilingualism and rights/ conflicts}

One myth of multilingualism is that it is nationally divisive. Modern states have been averse to acknowledging the existence of minority languages within their borders, and even less willing to officially grant and acknowledge speaker-rights to indigenous language communities, fearing that languages would compete for the same space in the same language ecology, and that this competition would exacerbate ethnolinguistic conflict and create demands for ethnic separatism. In this conception, the natural order of things is for a united and single territory to be perfectly covered by a single language. There is also the presupposition of a strong link between ethnic identity and a specific language. Regulating multilingualism and language contact is, in practice, an important way of policing the internal space of the nation state.

In contradistinction to this view, is the claim that one of the prime causal factors in interethnic conflicts is precisely the denial of linguistic human rights to a linguistically distinctive group, and that "linguistic affiliation is a rightful mobilizing factor in conflicts with multiple causes where power and resources are unevenly distributed along linguistic or ethnic lines" (Phillipson \& Skutnabb-Kangas,
1995: 496). Also, "the problem is not that ethnic groups are different, but the problem arises when they are no longer allowed to be different" (Hettne (1987: 67). In this view, granting linguistic rights diminishes conflict rather than augmenting it.

Different ways of managing multilingualism can also be seen in relation to the conception that states hold on how to police their nation state borders. In many countries in Africa, neighbouring states share languages that are spoken on both sides of a border, socalled transborder languages. However, the fact that two states may share a language seldom has any practical implications for their policies towards this language. Even if transborder languages are recognized, there are few, if any, regional concerted language policies that cultivate them. One implication of not giving transborder languages the same treatment on both sides of a border is that one and the same language will gradually split into different varieties over time, and this will ultimately lead to linguistic hinders to the easy flow of people and goods across borders. Language management is one more instrument with which to manage international relations and population movements, similar to monitoring and regulating by means of passports and tariffs.

There are alternative philosophies of border management that conceive of boundaries between nations as meeting grounds for populations, goods and services. Policies should address how to enhance cooperation across boundaries and how to simplify the flow of contacts. The shared cultivation of transborder languages between nations could be a significant way of contributing to a new regional geopolitical order in the spirit of open boundaries, 


\section{Multilingualism and the individual}

Ever since the inception of psychological studies on multilingualism, bilingualism has been associated with disadvantageous psychological, social, emotional and educational consequences. This view was particularly common in Europe and USA around the turn of the century, when much research claimed that the waves of immigrants from southern Europe to the USA were of an inferior racial quality. However, it is not uncommon to find individuals today who still claim that bilingualism is negative.

A, now, classic study by Peal and Lambert (1962) put the lie to much of the work on which many of these negative conclusions were based. These authors showed conclusively that many of the early studies that showed negative effects of multilingualism were methodologically flawed, and that no certain claims could therefore be made on the basis of them. Most of the subjects that were studied in these investigations were immigrant children in their first years in a new linguistic environment. Their language proficiencies in the host community's language (often English) were still underdeveloped, but they were no longer acquiring their original mother tongues in a native speaking environment. The children were quite simply in the process of replacing their first language with a second; they were, so to speak, caught experimentally in the process of language transition, and they did not have a native mastery of either language. Not surprising then that they became drop-outs with abysmal language proficiency scores. How was it possible to assume that these children were bilingual in the first place? As Peal and Lambert point out, the subjects had been classified as bilingual on the basis of their foreign sounding surnames. Just as detrimental to the validity of the results was the fact that so-called "bilingual children" and monolingual English speaking children were unmatched for crucial variables such as SES, education and gender. It has been known for some time now that education or social background determine what type of language speakers have access to. In this case, the students came from poor immigrant working class families. Again, it is quite expected that they should show a less standard proficiency in English.

Peal and Lambert's own study was a major break with the previous research in the field. It demonstrated that bilinguals, when balanced, that is, of approximate equal proficiency on specific measures in each language, and matched for socioeconomic status and a number of other variables such as sex, and age that are known to influence language actually outperformed monolinguals on a range of measures, among which were measures of verbal and non-verbal intelligence - contrary to previous prejudice. The authors suggested that one explanation for bilinguals' cognitive advantages might reside in the fact that they were often exposed to more than one way of linguistically encoding a thought or referring to an object, which would trans-late into a more general flexible intellectual orientation.

Since that time, a number of studies have replicated the findings of Peal and Lambert's study in at least 30 different contexts and cultural settings (Hakuta, 1986, 1987). A range of other cognitive variables has also been studied. Ingeniously designed and highly controlled experimental studies of divergent thinking or creative think-ing, where subjects are required to find new solutions to problems using old materials in novel and original ways (Torrance, 
Gowan, Wu \& Alliotti, 1970; Bain, 1974), concept formation (Cummins and Galustan, 1974), Piagetian conservation and field independence (De Avila, 1979), ability to monitor cognitive performance (Bain \& Yu, 1980), have been carried out. These studies have all recognized the potential cognitive, social and academic returns that a balanced bilingualism may bring to the individual. Metalinguistic ability, that is the ability to think, talk and flexibly manipulate language in abstract, form-based, ways (the language skills involved in puns or poetry) has also been shown to be consistently favoured by bilingualism (Ianco-Worrall, 1972; Ben-Zeev, 1977; Cummins, 1978). And far from bilinguals being socially and emotionally disturbed, which earlier studies had purported to demonstrate, more recent and controlled research suggests that they may even exhibit a greater social sensitivity and emotional perception than monolinguals.

Similar findings have been reported from highly multilingual developing contexts such as India. Mohanty (1994) conducted a series of studies on bilingualism and cognition with large numbers of children from the Kond who comprised a relatively homogenous group with respect to factors such as socio-economic and cultural characteristics and identity. The children were monolingual speakers of Oriya, the dominant language of the region, (although they had acquired the language in a situation of language shift from their mother tongue, Kui), and bilinguals who had varying degrees of contact with the two languages Kui and Oriya and who were therefore at different levels of bilingual proficiency. Once again, a clear relationship was found in this study between measures of cognitive performance and metalinguistic ability and degree of bilingualism.

\section{Summary}

In this section, we have looked into some discourses that represent multilingualism as chaotic, disorderly, an obstacle to civilization and social order, and a danger to mental and emotional wellbeing of individuals. Monolingualism in metropolitan languages is the preferred norm. Discourses, or myths, such as these serve to mediate unequal power relationships. They provide the rationales for majority language hegemony, and help keep linguistic minorities relatively powerless. Through processes of projection, characteristics of languages and multilingualism are taken to define the traits of the speakers. In the next section, we will explore some institutional structures that serve to reproduce minority language communities as marginal, focusing specifically on education.

\section{Multilingualism in education}

\section{Introduction}

In previous chapters, discussion has centred on how power relationships become transposed into different types of multilingual practice, and the political nature of language choice and language shift. We have also touched briefly on how the form and content of multilingual discourses are determined by the historical and political contexts which frame them. In this chapter, we explore in some detail the workings of educational institutions in the social reproduction of marginality in language minorities.

Current structures of bilingual education are perhaps one of the clearest manifestations of how the evolving systems of national and transnational development in their encounter with local cultures and languages have given rise to 
a specific ideology and management of multilingualism. Sreberny-Mohammadi (1997) summarizes well the tensions and contradictions of the deep-rooted cultural impact of imperialism on education in the following words:

Educational provision, curricula content and teaching materials are thus important sites of inherited economic dependency, complicated policy making and deep-rooted cultural conflicts, which mainly center upon the language of instruction, or the nature of the 'knowledge' imparted, or the very purpose of an educational system within its national, socio-economic and political frameworks. (SrebernyMohammadi, 1997; 60)

A number of bilingual programmes from around the world provide clear cases of how the school mediates inherited economic and political dependencies. Hidalgo (1994: 186) referring to Mexico says, "...[b]ilingual education serves as an educational strategy directed toward the linguistic and cultural assimilation of ethnic minorities. Mexico has a rich history of bilingual education which is worthy of study as a model of assimilation of language and ethnicity". CerrónPalomino (1989: 27) on Peru claims that "Bilingualism does not support Quechua or Aymara; on the contrary, it erodes them: this is the natural consequence of the struggle between unequally equipped languages and societies".

The effects that power imbalances between languages may exert on bilingual programmes can be seen in the ways in which curricula are formulated, in what languages are chosen for what purposes, in the teaching methodology embraced and in the manner in which materials production is accomplished.

\section{Curriculum}

One effect of the dominance of metropolitan languages is that the content of many curricula is oriented towards metropolitan languages and values, and, in this sense, caters to a small élite in developing communities. We find a division of labour between metropolitan and local languages such that metropolitan languages are used to teach the 'serious' curricula, and local languages are reserved for use with more domestically oriented, less academic content, such as social knowledge, cooking etc. This is because Western metropolitan languages have always been regarded as better adapted for technological, scientific and educational uses, on the belief that indigenous languages are less complex and therefore less able to express abstract, referential, and logical thought. The practical timetabling and subject use of the metropolitan language and vernaculars is just one of the many ways that the ideological message of vernaculars as 'less useful' is reproduced. Many factors conspire to heavily tip the scales against employing local indigenous languages in the classroom. There is the paucity of materials in local languages, their lack of availability, and the tendency for teachers' guides to be written in the metropolitan language. Taken together with teachers', often, shaky proficiencies in the languages and literacies of instruction, as well as the professional image of the teaching cadre as knowledgeable and proficient speakers of metropolitan language, it is hardly surprising that local languages are disfavoured.

When mother tongues are introduced into formal education, it is in order to teach old, mainstream curricula in new languages of instruction. Furthermore, the most common solution today to incorporating minority languages into the curriculum 
is to use them only for the first years of a transitional bilingual programme. The emphasis on metropolitan languages as languages of instruction at higher levels may negatively influence the attitudes towards the use of local languages at the lower levels. The fact that the metropolitan language is introduced as a teaching medium in grade 5 or 6 may mean that teaching at the first four years of the primary level becomes geared to managing this transition at the cost of teaching in the local vernacular. Concretely, this may mean that teachers ignore the local language aspects of the curriculum and focus on teaching the metropolitan language, and that subject content teaching be-comes yet another forum for second language teaching of vocabulary. Frequently, the national/minority language will not be an examinable subject, due to technical problems in norming and standardizing these languages for assessment purposes. This further contributes to their perceived lesser importance in the school day.

Local languages that are not used at higher, post primary levels of schooling, will obviously not be cultivated for these uses, which further impedes the development of educated varieties and registers that could be used to generate reading materials and to reinforce pupils' writing and reading skills. This in turn impacts negatively also on the primary school level; when there are fewer materials available, certain subjects will not be adequately dealt with in the local language.

\section{Languages of instruction}

Because minority languages have been actively barred from anything but a minimum contact with educational institutions, much work remains to be done in order to bring them 'up to speed' so that they can be used in such contexts. Their vocabulary, registers and stylistic resources often require radical expansion (intellectualisation). But it is also often necessary to grant them a more official status (officialisation) and even, perhaps, to unify different varieties under a common orthography. This process of standardization is yet another way in which the varieties spoken by local communities are implicitly signalled as of lesser value.

Authors have commented in detail on some of the side-effects of corpus work on minority languages. Hornberger and King (1996) note that one of the problems connected with standardization is that speakers may oppose norms that differ from their own practices, and that they may conceive to be "inauthentic". Standardization also often tends to marginalize speakers whose practices of language differ from the chosen norm. Intellectualisation allocates new functions to minority languages. This invariably results in the conferring of new forms and uses of the language at the interpersonal and societal level, which may give rise to splits and conflicts in the minority group itself. Officialisation of language may remove control over the language from its traditional speakers, which may further serve to alienate the community from its linguistic heritage. Finally, all of these techniques 'remodel' the minority language in the image of the dominant language; minority languages come to be inscribed with the social ideologies, class differences and standard/non-standard distinctions common to metropolitan languages. This is tantamount to nothing less than social engineering by means of language policies.

What this all means is that seemingly 'mere' technical considerations in adapting languages to school use carry great social and political weight, and 
impact upon matters of social and individual identity. Not even mundane technologies of linguistic description can be extracted from issues of power and marginalisation.

\section{Materials}

Another consequence of the global subordination of local languages is the lack of an indigenous production of materials. Part of the problem of ensuring an adequate supply and coverage of local materials has to do with the marginal status of minority languages. This means a low economic yield on teaching materials produced in these languages, which in turn means that publishers have little to gain in producing them commercially. Markets for materials in metropolitan languages, on the other hand, are more succulent, providing a motivation for major international publishing houses to out-compete local publishers in the production of materials in metropolitan languages. This causes a number of problems for educationalists. A few years ago, a Portuguese foundation produced materials in Portuguese as a second language that were designed for use by speakers of Portuguese varieties all over the world. These materials made no pretence to address the specific learning concerns of the millions of culturally and linguistically distinct communities that were supposed to use them. Generally speaking, metropolitan materials produced by Western or Northern publishing conglomerates means that local voices will not be adequately catered for.

Materials may both encourage and permit diversity in classroom practices, as well as help structure the teachers' classroom interactions. However, when textbooks are not available, an enormous responsibility and pressure is put on the teacher as the sole source of pedagogical innovation and progress (cf. Arthur 1994).
Without available minority language materials in all subjects, teachers will need to produce ad hoc translations or adaptations of metropolitan terms and concepts for their different disciplines. The frequency of use of the metropolitan language, and its visibility, is thereby increased in domains that were officially reserved for the transmission of content in minority languages. This contributes to the general message conveyed about these languages, that is, that they are not adequate for educational use. Inadequate materials also impacts on pupils in the sense that they hinder independent learning. In Botswana schools at secondary level, for example, only $12 \%$ of the pupils' time is spent working with textbooks (cf. Arthur, 1994). In general, without sufficient materials, teachers face great difficulties in adapting the curriculum to local conditions.

To the extent that materials do exist in indigenous minority languages, these have frequently been produced through translation of the mainstream, metropolitan curriculum. Although translation cannot be avoided, it does pose a number of problems that need a more focused attention. Translations that have not been thought through from a sociocultural perspective may introduce concepts, types of explanation, and even genres or registers that are non-indigenous to the community. This may provide a sound innovative resource for the languages, but it may also contradict indigenous ways of encoding knowledge in language. Translators need to be aware of their possible role in the globalisation of Western discourse patterns. More generally, minority speakers may not perceive this type of non-locally developed materials as relevant or meaningful.

Another problem is in the way in which translations are technically carried 
out, for example, in the formation of new words. Principles for terminology development or coinage in local languages may not accord with grassroots usage or that which is already in more general use. Finally, translation may hamper the development of innovative linguistic resources to cope with the expressive demands of subject materials at the community level in the languages concerned.

\section{Teacher training}

The weak position for local languages with their concomitant lack of status also impacts negatively on teacher training. Once they have been recruited, it is not uncommon to find that teachers may be actively discouraged from working in other tongue or bilingual programmes. Or they may discover that their career opportunities are tightly linked to metropolitan language criteria, that is, quite simply how well they speak, for example, English or Portuguese. On a more personal level, they may feel that their ideas and conceptions on what the transmission of knowledge means may conflict with local epistemologies and theories of learning and authority, or with the perspective on knowledge transmission that they have learnt at college. There are a host of social and economic problems that worsen the situation.

The teaching role is made more difficult by having to work in educational establishments ostensibly often geared towards themaintenanceand propagation of metropolitan values. This may also bring about role conflicts of various sorts. Given this, it is not uncommon that only the less ambitious teachers get put into bilingual programmes. Finally, teachers are a socially mobile segment of the population - at least in rural areas. Many strive for a middle class identity and set of values that is linked to the symbolic capital of mastering a metropolitan language. A strong focus on local language programmes may imply a redefinition of their status that they find unacceptable.

Komarek draws attention to the important role that teachers play in developing contexts, by saying that "[i] $\mathrm{n}$ [these] countries, not the curriculum is the basis of the educational system, but the teacher" (Komarek, 1996: 20). The teacher makes the curriculum "by doing'. Despite this, surprisingly little has been done in many developing contexts to adequately recruit or properly prepare teachers for their difficult role as 'transmitters' of local cultures and languages. Frequently, teachers are untrained in methodologies of bilingual teaching, specifically in mother tongue teaching, and appropriate techniques of second language teaching. In fact, they may not even be literate in their native languages. Or, even worse, they may not be speakers of the languages they have been sat to teach.

\section{Methodology and classroom language use}

In the previous section, we have noted that attempts to use mother tongues in school are plagued by poor materials' provision, insufficient teacher training in bilingual and bicultural methodologies, and conflicts between teacher roles, institutional requirements and social identities. Taken together with conceptions of schooling as expressed in curriculum content, these predispose for the unmarked choice of the metropolitan language as the main language of daily instruction. All this conspires to seriously limit the range of teaching methodologies that teachers can employ. In practice, pedagogy is 
often teacher centred, built around rote learning, with few creative classroom activities and a predominant use of the metropolitan language. Of course, the fact that teaching is so extensively oriented to use of the metropolitan languages that few pupils master is an important reason for the monotony and rigidity of classroom methodology; when pupils cannot understand and respond in cognitively adequate ways, much communication in the classroom takes on a semblance of a ritualised character. However, teacher-centred pedagogies of this type may also often function as an identity 'shield' for teachers who may experience linguistic insecurity with the metropolitan language or mother tongue. Teacher manuals may also be implicated, in that they may encourage a stricter teacher centred pedagogy as a way of compensating for lack of appropriate training in more pupil centred and bilingual teaching techniques.

In most multilingual classrooms, teachers report that, in theory, they shy away from using both languages in teaching, citing the prescriptive bias against mixed codes, and an old-fashioned linguistmentalist stance that the simultaneous use of two different languages may cause 'cognitive confusion'. In practice, the need to keep some semblance of order and learning in the classroom often forces the teacher to resort to using translation and code-switching to get her message across. Addressing this dilemma in Botswana schools, Arthur (2001) says:

The teachers I observed operated under conditions of tension between institutional pressure to adhere to language policy, that is, the exclusive use of English in the class-room, and their professional and personal instinct to code-switch tin response to the communicative needs of their pupils. (Arthur, 2001: 61)
There is one set of problems with teaching methods that can be traced to the fact that the bulk of knowledge production takes place in first world contexts. Instructional paradigms for first and second language teaching have mainly been developed on the basis of research conducted in the Western and Northern hemispheres, which differ socially, culturally and sociolinguistically to Third World contexts. Because second language learning can be seen as a process of "becoming a member of a speech community, governed in the same way as other forms of verbal behaviour by norms that obtain in that particular community" (Muysken, 1984: 101), teaching of metropolitan languages needs to consider local conditions and be based in local and situated methodologies. According to Kachru (1985: 23), it is necessary to reconsider "claims for the universal applicability of particular approaches for teaching and learning [English]. Likewise, Pennycook (1998), an acknowledged authority in global English teaching, comments insightfully that

[t]he history of the ties between ELT [English Language Teaching] and colonialism has produced images of the Self and Other, understandings of English and of other languages and cultures that still play a major role in how English language teaching is constructed and practiced: from the native speaker/ non-native speaker dichotomy to the images constructed around English as a global language and the assumptions about learners' cultures, much of ELT echoes with the cultural constructions of colonialism. (Pennycook, 1998: 19) 


\section{Summary}

Mühlhäusler (1996: 267) has noted how education is a primary vehicle for a knowledge flow from Western developed countries to the rest of the world. This implies a simultaneous flow of concepts, discourse patterns, and registers typically layered into metropolitan languages. $\mathrm{He}$ claims that mass education like mass literacy can only be administered economically if given in the smallest number of languages possible, particularly at levels above the first couple of years in primary school. Clearly, these types of institutional dynamics can be expected to reflect negatively upon bilingual programmes. Compared to the global markets and resources available to metropolitan languages, the opportunities available to minority language communities to develop and cultivate their linguistic and cultural diversity within educational institutions are not at all advantageous. Furthermore, a lot of teaching in mother tongues will, for a variety of reasons mentioned here, necessarily have implications for the cultural and linguistic integrity of the minority community.

In this section, I have explored some ways in which educational discourses have transformed relationships of power and dominance between different national and metropolitan languages into differences in number of hours each language is taught per week, quality of teacher education provided in each language, and scarcity or availability of material. By constraining the 'institutionally permissible' ways of inter-acting around, with and in relation to different language(s), traditional forms of curriculum design, materials production, teacher training and language description have all had the effect that indigenous languages have been disadvantaged in relation to metropolitan languages.

\section{UNDERSTANDING \\ LINGUISTIC MARGINALITY}

\section{Introduction}

The ideologies and institutions implicated in contact create types of multilingual practices that marginalize and disempower minority language communities. It should be clear by now that the topic of minority languages in education is far from just an educational issue. How can minority language marginalisation be conceived from the point of view a practical politics of language? More generally, what is the political nature of minority speakers' disempowerment? The search for an answer to this question is not just an academic exercise, as the way we understand the political nature of minority speaker marginalisation will have a bearing on the nature of the remedies we search for.

\section{Redistribution and recognition}

One way of understanding the issue of marginalisation is in terms of the age-old redistribution and recognition complex. Linguistic marginalisation (as well as language loss and shift) are part and parcel of other types of 'unsustainable development' that local, indigenous communities are exposed to. Groups that are marginalized are simply just not recognized as having legitimate linguistic claims; in this sense, they are stigmatised and this is because of their lack of economic resources and lack of power. 
On the other hand, the lack of resources and power is precisely one consequence of the lack of recognition on behalf of powerful, resourceful groups. For redistribution of resources to come about, including symbolic resources, not only must the existence of minority groups themselves be acknowledged, but it must also be conceded that these groups are disadvantaged and marginalized. The catch is that in order to be acknowledged, minority groups must have the resources, material and linguistic, to make their voices heard in the first place. However, marginal groups do not have the symbolic capital they need to succeed in society, or the social or other capital with which to ever access this symbolic capital. Marginal language groups are thus an example of, what Fraser (1995), in an incisive article in the New Left Review, calls a bivalent collectivity,, that is, "neither [socio-economic maldistribution or cultural misrecognition] is an indirect effect of the other, but [...] both are primary and co-original".

This framing of linguistic marginalisation suggests that issues of bilingual education are best understood in terms of a larger political framework of distribution and recognition. This is supported by the observation that those communities most at risk of marginalisation and linguistic/mother tongue disempowerment are those subjected to gross unsustainable development. The marginalisation of indigenous languages is part of the larger global process of unsustainable development which is causing deforestation, the biodiversity crisis, loss of land through soil erosion and pollution, global warming etc. (Nettle and Romaine, 2000: 158; see also Stroud, ftc. a and b, on marginalisation of minority groups). Minority communities are caught in a spiralling downward process, where nonsustainable development and exploitation on behalf of indigenous majorities or global industries increasingly deplete the natural and human resources on which minority groups depend for their existence, simultaneously weakening the cultural, linguistic and social capital of indigenous minorities. Bilingual programmes are part of this broader set of undemocratic processes which deny minority communities the resources they need to make a living, and educational issues therefore need to be discussed in a politics of redistribution framework.

The political nature of the bilingual education is also supported by a glance at the types of real-world activities and contexts that surround language revitalisation in minority language contexts. Issues of language are closely intertwined with 'welfare' debates, as seen by the fact that questions of language are topical issues for a range of political debates running in Africa today. For example, major political stakeholders are becoming increasingly more sensitive to the idea that the successful adoption and dissemination of new technologies depends on the use of local languages, as only these can reach out to the general population. This awareness has directed attention towards finding ways of conserving multilingual ecologies and promoting the use of transborder languages and lingua francas. In many countries, the realisation that the attenuation and demise of indigenous vernaculars is a direct reflex of the exclusive use of metropolitan languages as languages of power and prestige has intensified demands for the use of African languages at all levels of administration. In some countries in Southern Africa, language-based social movements are challenging civil society to re-dress the ills that oppressive language policies have for decades inflicted on minority language populations. These movements 
are producing exciting new discourses of language resistance, centring on the notions of language rights and language resources (e. g. Crawhall, 1998).

These examples illustrate the entextualisation of local languages into important and more general discourses of power and social action. Language issues are debated as central to economic, social, political and human rights' issues in Africa. This means that approaches to language that linguistically empower minority language communities must be part and parcel of more inclusive political discourses concerned with the political, economic and social empowerment of marginalized language communities. Any change in the linguistic fortunes of a minority language is the result of a close symbiotic link between language activism and political action and collective choice illustrating the bivalent nature of linguistically marginalized communities. In other words, minority language speakers need to attend to the ideological, economic and social conditions that determine their lives, and work in concerns with the expressive resources of language into these debates.

\section{Framing policies for bilingual education}

The above reasoning suggests that an appropriate approach to education in and through indigenous languages is one that proceeds from a politics of multilingualism that is an integral part of a more general ethical and socio-economically just politics (Hamelink, 1997; Skutnabb-Kangas \& Phillipson, 1995). The emphasis on an equitable politics of redistribution as a basis for programmes of language education carries strong implications for an emphasis on mother tongues. In contradistinction to the argument that access to resources requires English or some other global language, redistribution directs our interest towards the importance of mother tongues (see below on access to English argument). How, more precisely, is language education related to social, economic, political, cultural and individual development? An answer to this question is tantamount to examining in what ways local realities and local priorities can inform policy decisions.

\section{Social and political equity}

The promotion of multilingualism can be one powerful means to return power and control over the lives of subordinate communities to the communities themselves, both from the perspectives of politics and ideology, as well as economically and culturally. Language education should therefore focus on ways to equip speakers to strategically use language for their individual and community empowerment, and to develop their multilingual life-spheres. Following de Beaugrande (1997), language education should support 'discursive strategies for inclusive, creative and self-reliant communication" and "welcome multiculturalism as an opportunity for actualising the widest range of human potential" (p. 113, quoted in Bruthiaux).

\section{Enrichment of cultural and \\ linguistic identities}

We touched on the importance of multilingualism for the everyday constitution and expression of multiple identities. And we underscored how, on the individual level, knowledge of multiple languages has been found to be emotionally, socially and cognitively enriching. A perspective on language from the vantage point of poststructuralist theory as one articulation of discourse in the Foucauldian sense 
of the term, that is, as "practices that systematically form the objects of which they speak" (Foucault, 1972: 49). Discourses are networks of convention, knowledge and practice that determine how one perceives and behaves in the social world. The ways in which we subjectively live our lives, giving meaning to the material social relations in which we live that structure our everyday lives, depend on the range and social power of existing discourses, our access to them, and the political strengths of the interests that they represent (c.f. Weedon, 1987). In this concept, language offers a historically specific, socially determined, competing and complementary range of ways of giving meaning to social reality, thereby articulating different "lived relations" to material life (Althusser, 1971). Cameron (1990: 90), notes that "[c]hanges in language use change the repertoire of social meanings and choices available to social actors", remarking at the same time "language-using is a social practice in its own right".

\section{Economic development}

Far from being economically disastrous, multilingual networks may contribute to the creation of wealth in society, especially among those subordinate groups who are traditionally excluded from official labour markets and sources of income dominated by the metropolitan speaking elites. There is evidence that literacy rates correlate with indices of economic development such as child mortality; vernacular literacies counteract this.

Furthermore, there is now ample evidence that education levels correlate with economic and social benefits. There are numerous studies that show the effects of education for earnings (Liue, 1988; Foukhouser, 1999; Bedi \& Gaston, 1998) and some work that more specifically looks at the effects of language education on economy (Rassool, 1999).
Furthermore, sustainable development in the sense of non-depletion of world's resources requires more participation of indigenous peoples applying indigenous and traditional forms of knowledge to economic development. This is best accomplished through use of local languages.

\section{Democracy and regional development}

We also recognized the role that multilingualism can play in minimizing ethnic and regional conflicts. Providing discourses of power and authority that would be accessible to more people. Counteract effects of globalisation. We noted that the use of multiple languages creates order in society.

\section{Educational efficiency}

One of the most important implications of this conglomerate of interrelated factors pertains to educational efficiency. There is a clear link between power, disadvantage language and educational efficiency. A sense of language that ties into a praxis account and that has educational relevance is language as discourse and an expression of community of practice. Language is perhaps the single most important visible factor in educational efficiency. Snow (1990) reviews a study by Collier (1987) and Collier and Thomas (1988), which shows how learning to read in a language one does not speak well can have long term consequences for academic achievement. McLaughlin (1986), reviewing research by Rehbein (1984), says:

The ability of Turkish children to deal with complex texts in German was affected by their ability to understand these texts in their first language. Rehbein's investigations suggest that there is a strong developmental interrelationship 
between the bilingual child's two languages and that conceptual information and discourse strategies acquired in the first language transfer to the second. (McLaughlin, 1986; 34-35)

Cummins in numerous studies has formulated the interdependence hypothesis to account for this type of dependence and transferability of skills across languages. His formulation of the threshold hypothesis, provides a powerful theoretical accounting for the impact that different individuals' bilingual proficiencies have on intellectual and cognitive measures. This latter notion refers to the idea that language competencies may be related to different thresholds of proficiency - a lower threshold that individuals must reach in order to avoid disadvantages from language contact, and a higher level that must be attained in order for bilingualism to confer cognitive advantages. However, what is imperative to keep in mind is that language is merely a mediating factor that translates the material and political powerlessness of marginal communities into educational failure. Remedies that redress this primary ill need to be in place in order to then aim for educational efficiency.

Another important variable in educational efficiency is teacher quality - teachers' knowledge of subject matter, pedagogical practices and competence in the language of instruction. Teaching reading is more effective when teachers emphasize under-standing what is read.

\section{Summary}

An adequate conceptual understanding of the way in which issues of language and multilingualism relate to matters of poverty and disempowerment for minority language speakers is a necessary prerequisite for the formulation of sound policies. One conceptual framing of the problem of multilingual education informs us that by 'empowering' languages, we may empower speakers. And on the other hand, empowering speakers empowers languages. The main implication is that thinking and policies on language provisions must be devised that address both more strictly language educational issues as well as policies that address the larger sociocultural matrix in which these issues are embedded. In designing multilingual education, the emphasis needs to be on how to make language relevant to the social, political and economic empowerment of the individual and the community. Furthermore, considering the fact that we are dealing with questions that are fundamental to the politics of identity and redistribution of (language) capital, policies - with respect to how they are formulated and their content - need to attend to questions of democracy and participation. The recognition of the value of multilingualism in the local life spheres of individuals and marginal communities requires the active participation of these local communities in the ways in which multilingualism is done educationally. We have had frequent occasion to note how global power relationships are reproduced in minority language policies. In particular, bilingual education should attempt to address how the local needs of minority populations can be met in the face of transnational developments of both a linguistic and non-linguistic nature.

In these terms, the overriding goals of a policy of language for education must be to

- contribute to increasing social and political equity for all indigenous minority groups.

- contribute to the enrichment of 
cultural and linguistic identities of individual and group.

- help ensure economic development of minority communities.

- contribute to an awareness of the role of language in democracy and for regional mobility and peace and conflict reduction.

- contribute to educational efficiency.

- contribute to minority groups' social and linguistic management of global change.

\section{PRINCIPLES OF EMPOWERMENT THROUGH MULTILINGUAL EDUCATION}

\section{Introduction}

The discussion in the preceding chapter raises the theoretical issue of the relation of linguistic empowerment to material, economic and political empowerment more generally. And it also pinpoints a further concern, namely the role that language education can play in empowering communities with, and through, language. As noted in the previous chapter, issues of language education would benefit from being framed within a more wide-ranging socio-political discourse on language and community empowerment. In short, language education needs to address the linkage between economic and material (re)-distribution and political participation, on the one hand, and ethnic and linguistic identity and language resources, on the other. However, developing language programmes for minority communities should not be just informed by these wider concerns, but the actual proofs of designing and implementing a programme should also comprise one of the main remedies that marginalized speakers can rely upon in order to pursue a more equitable 'balance of power'. What does all this mean in practice? How should we conceive of language programmes in order to reach these goals?

This chapter elaborates in some detail on the general policy directions staked out earlier by suggesting a number of design features or principles that could serve as input to determine the form and content of multilingual education. The presentation purposely avoids using the notion of programme, which leads thoughts naturally to the idea of a self-contained and complete pedagogical package, and overemphasizes educational solutions to multilingualism. Besides this, the very diverse nature of the contexts for multilingual education will inevitably mean that there are different ways of reaching educational goals. Also mitigating against a set programme is the dictum that local communities themselves should have a major input into decision making at all levels. An alternative to the notion of a programme is the idea of principles for multilingual education, which can be seen as design features that can be met in many different ways depending on context, ethnography, culture and tradition, and which can just as well be implemented in 'ordinary', non-bilingual classrooms as in special programmes. Twelve such principles will be briefly presented in the remainder of this chapter.

\section{Twelve principles for educational language provisions in multilingual settings}

Principle 1. Community control or ownership of bilingual programmes, and local inputs into, and community management 
of, the bilingual programme should be maximized.

We have noted in previous sections how the management of multilingualism, in society generally, as well as in policy making and education reflects global and local relationships of power. It is also apparent that poverty, marginalisation, and disempowerment of minority groups are created and reinforced by the way societies manage multilingualism, both ideologically and practically. One aspect of the problem is that in many developing contexts, educational language policies are decreed in a top down manner, rather than taking the needs and opinions of grassroots into account, frequently serving to articulate the power interests of the national elite. Tollefsen (1991) in an important comment has said that

language planning policy means the institutionalisation of language as a basis for distinctions among social groups (classes). That is, language policy is one mechanism for locating language within social structure so that language determines who has access to political power and economic resources. Language policy is one mechanism by which dominant groups establish hegemony in language use...Hierarchical social systems are associated with exploitative language policies, that is, policies that give advantage to groups speaking particular language varieties. (Tollefsen, 1991: 16)

There is evidence from a range of contexts (Papua New Guinea, New Zealand, Aboriginal Australia) that the participation of local community in the design and implementation of programmes contributes to a more successful language provision. Fettes (1998: 145) suggests that a fundamental prerequisite for educational use of indigenous languages is "an acknowledgement of the importance of community ownership and the centrality of the home environment". In fact, Fishman (1985), commenting on the role of the school in maintaining languages, says that

[n]owhere in the world have major programmes of language maintenance, language revival or language revitalization succeeded if their major 'bet' was on the school rather than on other, more primary, social processes [...] the difference in community structure, in community control of its own residential and economic bases, and in community regulation of the domains and degrees of interaction with [AngloAmerican] are the crucial differences that obtain $[. .$.$] school will have its$ role to play in the overall language maintenance design, but it will do so serving a vibrant and purposeful local community. (Fishman, 1985: 374)

As the school is embedded in the local community, the interaction of school and community ought to permeate the structure, process and implementation of multilingual schooling. Penny and Edmondson (1998) refer to how community driven literacy programmes in Canadian Inuit and Cree communities led to the attainment of high levels of literacy. As early as 1770, Moravian missionaries in Inuit communities incorporated literacy training into family and elder based education, where it was treated as a life skill and taught by family elders. In another community context, the success of literacy in the Cree syllabary was reached "without the benefit of schools, without the benefit of trained teachers and without the context of print-literature" (p. 2). The 
authors suggest that the success of these programmes was due to the fact that they took place with "the support and active promotion of the customary system of education within the community and ...were culturally appropriate" (ibid.), and that community members did not see literacy as part of a major cultural disruption. La Belle (2000) writing on the changing nature of non-formal education in South America has also pointed to the importance of the local community for literacy promotion, as well as facilitating finding solutions to problems of health, nutrition and political choice.

Both policy-making and implementation need to be opened up to extensive democratic participation of grassroots organizations. The requirement that community members themselves become involved in the development of bilingual programmes, and that the institution of the school be directly answerable to the needs and desires of the community would contribute to the empowerment of local communities.

The spirit of community management also suggests that the structure of policy-making and implementation should be such so as to accommodate conflicts of interest between local, provincial and national structures of decision making. It involves decentralizing power and control over bilingual programmes, developing a concept of community ownership and increasing the autonomy of bilingual programmes vis-à-vis national systems (see also below, 10. 4, for an extended discussion of this). The involvement of local communities in multilingual education would most certainly have a range of implications for the development of relevant curricula, locally authored materials, and culturally appropriate language learning and teaching methodologies. One of these implications is that they can all be designed with the sociolinguistic, economic and political conditions of the community in which the programme is sited as a point of departure. Refinement and adaptation of decisions on the basis of local needs and resources would contribute to sustainability. The greater involvement of local community representatives in very early stages of the process of creating these infrastructures as with policy making generally, may also help to make language pro-visions more affordable (by getting rid of the waste of programmes not appropriate to local contexts) as well as increase the confidence of the teacher.

Principle 2. Language provisions for minority communities should emphasize both maintenance and development of local languages and metropolitan languages throughout the entire educational system.

We have already noted a host of good reasons for this principle. First of all, concerns of social equity and democratic participation imply that indigenous languages should be used and developed in all relevant contexts. Secondly, as we have seen, programmes that emphasize a notion of schooling as fundamentally oriented to transmission of knowledge through the metropolitan language alone fail for a range of reasons. Given the reasoning and justifications that we have referred to above, the only viable solution is to extend a well-qualified intellectual use to indigenous languages throughout the child's entire school career.

A stronger emphasis on knowledge transmission through mother tongues implies that indigenous minority languages must be used for teaching relevant subject content at all levels of education. Instruction in a language must be clearly integrated with content instruction. It is essential that content 
instruction does not become reduced to teaching vocabulary in some language, but that appropriate means be used for doing science or mathematics or history in the language. This is essential so that parents and teachers perceive that time spent on indigenous languages is time spent on teach-ing valuable subject content.

One important way of achieving coverage of relevant knowledge is to integrate school subjects with local activities, e.g. teach concepts of science through local technologies. In some cases, it might be necessary to carefully prepare community members for this. Previous attempts to do something similar has shown namely that parents feel as though teaching local technologies in schools reflects a second rate education, and brings back memories of the vocational schools of many colonial education-al structures. It may also be the case that the association of schooling with book learn-ing may mitigate against learning in real world contexts. Parents and teachers may also feel that the school is competing with the community in areas (such as craft production) that the community may do better. There is also some evidence that suggests that specific ways of incorporating local content into classrooms contributes to the marginalisation of local languages and the disempowerment of their speakers. In other words, some thinking might be required in order to find workable ways of incorporating local technologies into school curricula.

Subjects taught through indigenous languages must be treated in the same way as subjects taught through the metropolitan language. examinable subjects. If there is a system of evaluation and testing in the official language, then there should be a system of evaluation and testing in national languages. This will increase the motivation to teach in and about these languages.

Appropriate secondary discourses in mother tongues need to be developed for this purpose. Gee (2000) underscores how "different material circumstances have deep implications for one's access to different social positions in the knowledge economy of the new capitalism", and how this impacts upon, and is mediated by, the codes used by social actors. The challenge then is how to construct secondary discourses relevant to schooled learning around the primary socialization routines of child-caretaker. Gonzalez (1993) has underscored how the intellectualisation of languages also comprises a necessary condition on their perceived usefulness as languages of instruction. One suggestion is therefore to focus language cultivation on higher academic levels, which would provide an 'academic target' for teachers and pupils to work towards, and will have the effect that registers and vocabulary will cascade down to the level of primary education. Higher-level registers and vocabulary also permit the language to be used in complex daily adult activities, thereby enhancing its value to the community and their willingness to invest in its development. The academic development of indigenous languages will most certainly enhance the political and participatory capital of their speakers.

Principle 3. Language provisions in multilingual contexts should be structured around the notion of language portfolio.

Recognition of multilingual networks is, as we have seen, important for economic and political reasons, and access to multiple languages allow 
the expression of important individual and group identities. Furthermore, a policy that attempts to link language in education to economic development and democracy, as well as to regional peace and stability, demands that multilingualism be taken into account, as local and regional economies, and the democratic expression of voice are tied to the languages that people use. One problem with many bilingual programmes, however, is that they ignore the multilingual complexity of their clients, focusing often on one or two major local languages and the metropolitan language. In other words, educational programmes do not see the areas in which they work as linguistic ecologies. This parsimonious view of bilingual education is encoded in, and reinforced by, concepts such as mother tongue, native language, second language, etc. - a taxonomy that represents a specific, monolingual point of view. In multilingual communities, speakers may have more than one mother tongue: a potential mother tongue in the sense of first learnt may not be the best mastered; a non-primary language may not be a 'second language' in the sense that it can be taught with typical 'second language methodologies. In other words, the set of assumptions laid down in the taxonomy may not at all apply, and may even bias planners towards pedagogical solutions that are not appropriate for the contexts.

A national policy, that deprives local minorities the use of the local languages, constrains the development of their linguistic resources and risks exacerbating community disempowerment. Difficulties in finding solutions to this problem, as with many other areas of development, result from the fact that centrally formulated language policies in developing contexts cannot "take account of the rich diversity of sociocultural realities, and have produced uniform schemes unsuited to local conditions" (Robinson, 1996: 40). The local or regional level, which is the socio-demo-graphic level at which people use their languages on a quotidian basis, may not host the same set of priorities as the national, government level. This, of course, is just one more reason to decentralize decision-making.

In line with this, multilingual education ought to consider building student competencies in more than one indigenous language, or language in daily use. The notion of language portfolio tries to accommodate the fact that speakers utilize the structured diversity and functional links that exist between many languages on an everyday basis in a portfolio of languages. It is designed to capture the individual speakers' solution to the regional or local spread and diversity in patterns of language use. Language provisions should be based on the idea of language portfolios of the citizens and find ways of managing this institutionally in terms of multilingualism as a resource, rather than a problem to be solved through excluding languages.

Commensurate with the concept of language portfolio is the possible existence in a community of a repertoire of literacies, multilingual literacy. Literacy is primarily a sociocultural process, and children are socialized into literate practices through a range of events in everyday contexts in which text, or talk about text and around texts, are at hand. Different literacies in different languages may carry very many different and complementary meanings for their users (cf. Stroud, 1994). Therefore, formal literacy learning should be in the language(s) that the child masters best and mirror the range of literacy types and practices that the community has literate traditions in. As with language, 
learning literacy is facilitated by being taught in routines and events that are culturally familiar to the child.

An orientation to language teaching based on the notion of language portfolio means that descriptive models of working with language need to be complemented with more sociologically oriented studies that explore the composition and meaning of multilingualism for local communities. In line with this, good quality sociolinguistic surveys that will capture community language use, attitudes and developmental trends in language vitality ought to be made available. For example, it is not certain that the mother tongues of children are the preferred or most appropriate teaching media in all cases. The sociolinguistic situation may be such that a second (national) language is learnt and used on a daily basis by the majority of a population.

In practice, multilingual programmes around the concept of language portfolio could be designed in such a way that they 'widen the students' linguistic experiences by progressive differentiation from local speech to supra-dialectal varieties, culminating in a sophisticated grasp of standard and literary styles for the community's motivated specialists" (Khubchandani, 1974). In other words, "work on languages in schools should correspond to the communication patterns among different speech communities prevailing in the region" (ibid.). Children should be made familiar with a wide variety of speech varieties, attuned to variation in usage depending on situational use. Instructional strategies in contact languages should be built on and envelope those interactional strategies that exist outside of the classroom. This means aligning school and out-of-school varieties of the same language.
The notion of language portfolio articulates well with prevailing trends in world markets and the growth of the knowledge-economy. 'Fordist' thinking, that is, the systems logic and mass culture of a world built to accommodate the standard production line of Henry Ford - in essence an attempt "to force the cultural simplicities of homogeneity on a world that was inherently heterogeneous" (Cope \& Atlantis, 2000).

Principle 4. Language cultivation should be conducted from a grassroots perspective and be a central strategy of political empowerment for the community.

One of the most pressing tasks facing many speech communities in developing nations is the maintenance and cultivation of indigenous languages. In many cases, this involves revitalization, "an attempt to add new forms or functions to a threatened language with the ultimate aim of increasing its uses or users" King (1999: 110). We noted earlier the political character of much language cultivation work. Language revitalization/cultivation is an activity infused with political significance. Jaffe (1999: 247) warns that "forms of language activation that reproduce a dominant language ideology also reproduce the structures of domination" and she draws attention to the "dilemmas, ambiguities and limitations of forms of resistance that do not challenge the premises of dominant models of power and value (ibid.: 247). In fact, revitalization is best approached as a medium and a tool for socio-political transformation. I have suggested that practices of language revitalisation require an approach to language that affirms local participation in new expert discourses as part of an empowering politics of language. 
Essentially, successful revitalization should aim to bring about changes in the structural and symbolic resources of a language through concurrently developing the political subjectivities and agencies of minority speakers. The main justification for this is that language revitalization needs to be part of a more general transformative politics in order to succeed, and that language reform by increasing the discursive resources and positions open to speakers is an important means of bringing about social and political transformations. However, a focus on language usage may produce changes in social meanings and empowerment of linguistic resources only under certain conditions. Cameron (1990: 90) notes "campaigns and debates specifically on the issue of 'sexism' in language were instrumental in altering linguistic usage”. Her example of feminist language illustrates an important point, namely that changes in linguistic repertoires come about when linguistic issues are highlighted, debated and contested in the context of more general political and social concerns. In the particular example she discusses, points of language usage are inserted into a more general public political discourse on gender issues. Gender issues are characteristically worked through on a number of different social arenas, and it is through the concerted efforts of groups and individuals to politically produce new resources and new social relations that change in linguistic repertoires and structural resources come about. By situating language questions in a wide range of discourses, both as a rhetorical tool and a politico-linguistic object, speakers also extend the range of alternative meanings available in a language. Cameron's (1990: 90) comments on change in sexist language is interesting in this regard: "historically speaking, there is certainly a relationship between feminist campaigns for equal opportunities and for non-sexist language, but the one has never entailed the other, nor did either just reflect the other". In this context, I have noted the need for speakers of indigenous languages to gain access to political platforms where language issues can be debated in conjunction with other political issues.

Cultivating a language is not a neutral, technical activity, but informed by ideological perspective and goal of the language planner. We therefore need to make decisions on how to conduct corpus work on the basis of socio-political and cultural priorities. For example, the choice of coining new terminology in a Bantu language such as Swahili through either loan adaptations or translations from English is not a neutral, technically linguistic, choice but a tactical one. The coinage of a new term following Bantu word-building principles will potentially have the advantage of the term being more easily adopted by other Bantu languages. Ethnic or minority languages themselves may also contribute linguistic materials to Swahili. The choice of an English term may risk contributing to the elite closure of a register or variety, keeping the general community of speakers away from full mastery of the terminology unless equipped with the proper background and training. Furthermore, the method by means of which terminologies are cultivated may have implications. Terminology development and the development of specialized registers and styles should be conducted in the field in those contexts where they are used and have a natural context of development. Professionals in the field should be provided with the necessary specialist assistance to evolve their own terminologies and registers. 
This means that local input to language terminology development could be in the form of fieldwork in factories and production units to see what words are used at the workbench (as Tanzania has done since $1970 \mathrm{~s}$ - in order to stave off the use of artificial terminologies).

The approach to revitalization advocated here means that speakers' own voices must be heard on issues of language. Because the way languages are debated and politicised not only shapes speakers' practices and ideologies of language (cf. Blommaert, 1999), but also their identities as language users, minorities themselves urgently need to recapture a voice in the political discourse on language. Minority speakers must be able to politicise their own knowledge and representations of language and language use; to decide on contending definitions of what languages are and what they may mean; to advance matters of language as relevant to and determinate of a range of social issues - policy issues and questions of equity - and to have these matters debated with due consideration to discourses on language. This means that they must have available ways of talking about language that can adequately represent and interpret the linguistic realities in which they live. However, much current theorisation of language and politics is often unavailable to those communities who are 'theorised', and, in many cases, fails to provide any clear basis for political action. We therefore also need to consider how discourses on language might be constituted that are able to represent these voices and articulate their multilingual experiences and subjectivities. In this section, I suggest one way in which discourses on language, as well as the material and social conditions of knowledge production, around language, could be transformed so as to provide a 'praxis theory' of language and politics.

One basic requirement for a praxis discourse on language planning is that it should offer an alternative to dominant ways of positioning indigenous languages in social and political space. An example that illustrates what this could mean in practice and, at the same time, underscores the importance of a 'grassroots' perspective' on language issues is the contrasting notions of linguistic variants and varieties entertained by experts and ordinary people. Descriptive linguistics maps out the boundaries and borders ofa 'language' on the basis of formal criteria; different structures for the same meanings are located in different language systems, and superficially different forms that can be argued to originate from the same pattern may be designated as lectal variants. However, experience shows that such a formal determination of language boundaries may not agree with speakers' perceptions of likeness and difference. It may also ignore or disguise the important sociosymbolic work that speakers accomplish when they construct, deconstruct and reconstruct borders between variants with the purpose of positioning themselves variably to other speakers. What appears formally identical to the linguist may carry powerful messages of difference for members of the speech community. Urciuoli (1990: 295) notes that "people build their sense of language around relationships as much as they build relationships around language", and these relationships may depend on the dynamics of race, class and gender as much as on ethnicity. The fluidity with which speakers view boundaries between variants in their languages tells us something important about the complex stratified and shifting communities that 
they live within, and the multiplicity of allegiances that they construct around perceived common variants. One important role of intellectuals and experts in a new discourse on language would be to authenticate different narratives of versions of language and culture such as this, by crafting novel resources and new social meanings into legitimate and authoritative language structures and repertoires. This would help capture precisely how speakers subjectively make sense of their lives as minority speakers, and how they linguistically articulate, embody and play out power relations on a daily basis.

Another facet of a new discourse is a wider and politically conscious construction of linguistics and language planning as a scholarly but also activist field of knowledge. In this context, current forms of social production and distribution of knowledge around language need to be transformed. The most vibrant debate on these and similar issues in the past decade can undoubtedly be found among practitioners of Applied Linguistics (AL) — a debate that promises to substantially transform how practitioners of AL both perceive and practice their fields. Even though language planning and revitalisation make up core areas of AL, very little of substantial or practical consequence from this debate has managed to filter down to these specific areas. And yet, the main themes of the debate are highly pertinent to the concerns that should be addressed in language revitalisation contexts, such as how to integrate personal, moral, social, political and historical factors into the AL research process (in topic selection, project design, fieldwork, analysis and dissemination), the meaning of research neutrality and how to deal with "pluralisation of authority" (Rampton, 1998: 249). Roberts (1998) has proposed quite a radical solution to the problem of how expert discourses recontextualise informants' multilingual 'realities'. She argues that AL should "use interpretative tools that connect with [...] the interactive experience, theories and metaphors of informants and practitioners" (p. 75), that by definition are responsive to participants' social engagement in the field. She claims that "application should properly be part of the intellectual work of the field and not simply a conduit down which theories are successively and reductively poured" (ibid.). This stance is clearly of prime interest for the question of how speakers of indigenous languages may lock their linguistic concerns into more established and authoritative linguistic discourses.

If a new language planning discourse is to include interdisciplinary discourses between researchers and practitioners as an important and integral part of its definition, how then to theorize the process of intellectual negotiation between these two parties? Rampton (1998: 3) sees AL "as a point of interdisciplinary synthesis where theories with their own integrity develop in close interaction with language users and language professionals", i.e. "a productive interdisciplinary dialogue between theory and practice" (p. 4). He points out that applied linguistics in this sense "integrates the investigation of fundamental linguistic processes with dialogue with the community and the professions" (p. 5). Roberts (1998: 68) suggests that "applied linguistics needs to constitute its intellectual base within practical work outside the academy rather than simply applying theory to practice". She notes how "practice' "has often been set aside as not part of the knowledge-making endeavor (emphasis mine)...and notes that [t]here is no sense of knowledge as constituting both an understanding of practice and the 
ability to conceptualize this practice". The point of view captured in these quotes is nothing less than a major shift of perspective and emphasis on the relation of experts to laymen, in that it refashions more traditional relationships of expert-advocacy into a partnership between minority speakers and linguists.

Principle 5 Language cultivation and bilingual programme development should, where feasible, be conducted on a regional basis.

In order to maximize individual access to multilingual networks and to contribute to regional peace and stability through free movement of peoples, language cultivation is most appropriately conducted on a regional basis. Strong regional cooperation would also help cushion the linguistic effects of globalisation, by reinforcing the 'strength' and position of local languages. It could also provide substantive arguments to counteract claims of too great a linguistic diversity among opponents to bilingual education. Regional solutions to language description may increase the constituency of languages by making them mutually accessible to large numbers of speakers through different ways. For example, communities in situations of language and culture contact employ different ways of extending their linguistic repertoires, and have developed diverse mechanisms for coping with language diversity, such as 'natural bilingualism' in the most vital language, translation brokers, etc. Transborder variants should be encouraged and lifted up.

Another way of making diversity manageable is through processes of harmonization of languages. In the ongoing debate in South Africa as to the status of two large languages Nguni and Sotho, Alexander (1989 and 1992) has suggested that a long-term process of standardizing written varieties of these two language clusters should be initiated. This process should be based on the structural convergences that have developed in the language systems over time as a result of intensive long-term contact between them. The suggestion has the advantage of unifying speakers and languages around a common norm. In this respect, the situation for Kiswahili in East Africa is extremely favourable for harmonization, as in Tanzania 90\% of the languages are Bantu languages, and in Kenya $75 \%$. A case in point is provided by the languages Chiyao, Chilomwe, Chisena, and Chinyanja (Chichewa) many of which are widely spoken in Malawi, Mozambique, Zambia and Zimbabwe, especially Chinyanja. There have been proposals that this latter language be developed as a regional lingua franca, which would require a harmonization of work on language descriptions. The development of Chinyanja would undoubtedly contribute to regional integration. There are already some initiatives in working along these lines. Networks of cooperation can also be built between the industrialized world and third world universities and language centre developments by using available technological resources. For example, trials have been made of parsing the Maputo Portuguese Urban Oral Corpus with a parser located in Aarhus in Denmark by means of the Internet. And Larry Hyman in the USA is coordinating a large computerized Bantu lexicography project, and this could also serve as a model for how such technological cooperation could be accomplished.

In order to promote regional cooperation, regional universities/ departments specializing in applied linguistics, multilingualism and education could be created, as well as interregional 
language commissions or boards. At least for Africa, where many languages are spoken cross-border, this would facilitate and provide the infrastructure for the getting together of researchers around the development of common materials. Apart from matters of language description, a programme of implementation geared towards the provincial, regional and/ or local development of a bilingual system of education requires a base of knowledge maximally adapted to the requirements of local actors and institutions - a form of grassroots, doit-yourself manual for setting up and consolidating programmes. Currently, there is very little in the way of available information on bilingual education programmes of sufficient quality that could be funnelled into a database and adapted to serve such a function. Due to the lack of available information on policy and implementation, data should be gathered from all contexts where bilingual programmes are running that could be used for flexible revision of programmes and for impact evaluation. This data could be in the form of information on enrolment, dropout etc that is reported to Ministry of Education statistics. Data could also be in the form of action-research protocols from teachers, principals and language committees monitoring the progress of programmes. Ultimately, a worldwide database should be constructed which all projects can hook up to. This base would provide basic information about projects/personnel/experiences; users will undertake to continually report back evaluations according to specified format for the expansion of the database.

There is of course a continual need for monitoring, information, revision and development work in setting up a bilingual programme on a regional, provincial and/ or local basis. An analysis of consequence to continually view the impact that the setting up of bilingual programmes has on societies along specific parameters should also be part of the development of bilingual language services. One way in which all this could be managed would be to establish a regional centre or university department that would work only with questions of multilingualism, functioning as consultant to communities, and open to all participating countries. One model for this type of institute can be found in the regional universities of Latin America.

Principle 6. Culturally sensitive teaching methodologies should be employed for both indigenous languages and metropolitan languages.

Local control also more easily permits the use of culturally appropriate routines in multilingual teaching contexts. Children's socioculturally determined norms of interaction, learning styles and individual and social identities need to come into play in a learning process, and using mother tongues or indigenous languages is the most important way of doing this. Such routines can make language and content instruction more meaningful for the child by building on community specific values and resources. A number of studies have shown how children from culturally varied backgrounds may experience problems in mainstream classrooms with respect to how teachers manage interactions and assume certain types of learning style (Au, 1980; Shultz, Florio \& Eriksson, 1980; Crago, 1992; Edelsky, 1994). For many children from marginalized language communities, schools are "alien institutions", where the norms governing behaviour, "the goals of the actors and the messages that are conveyed are often 
mysterious" (Snow, 1990: 63). Snow goes on to say that

[m]any studies have shown that academic achievement improves when children are provided with teachers (or even teacher aides) from their own language and cultural groups - adults whose expectations about how the classroom should be organized match the children's experiences, adults who understand and correctly interpret the children's ways of expressing themselves, and adults whose structuring of relationships and of learning contexts re-creates what the children are familiar with. (Snow, 1990: 64)

Using a language and cultural framework that children are familiar with removes the need for children to develop strategies to compensate for non-understanding, and for teachers to resort to mechanical face-saving and class disciplinary moves typical of classrooms where the language is unknown to the children (cf. Hyltenstam \& Stroud, 1993; 1996 for an exemplification of this from Mozambique).

One implication of the language portfolio notion is a different way of conceiving of language use in classroom, and applies specifically to code switching which is a communicative phenomenon that should be explicitly accounted for in decisions about teach- ing methods and language policies. One important facet of code-switching is that it is involved in the negotiation of secondary discourses - switching helps contextualise how the message is to be taken. Code switching should be a communicative resource for pupils, and there should be more tolerance of code switching in spoken and written discourse. Quite simply, more attention should be paid to exactly how teachers and learners get things done with two or more languages in actual classroom practice on a day-today basis.

The teaching of indigenous languages is frequently conducted by means of pedagogies developed for Northern language teaching contexts. For Africa, there is as of yet a lack of formalized experience on teaching African languages as first languages in classroom contexts, and very little knowledge of how to teach African languages as 'second languages' to speakers of other African languages. The same holds for other indigenous language groups. Teachers also need to be given methodological insights into how two (or more) languages can be used in bilingual classroom teaching formats, as well as what teaching a nonnative language in a developing and culturally very different environment entails. Teachers have generally a range of experiences, their own and those of their communities, on what constitutes a good, sound, pedagogy.

Local indigenous languages are not the only languages for which new methods of teaching need to be developed. A fresh look needs to be taken on the way in which teaching of metropolitan languages is done. We have consistently noted how even though metropolitan languages are often viewed as harbingers of wealth and opportunity, they tend to exert a hegemonic effect on communities of speakers from developing nations, and may serve to exclude many people from important social arenas. Despite this, a number of writers have pointed to the potential for indigenised/nativised forms of metropolitan languages to carry a critique and resistance to current socio-economic and political structures and trends in many third world societies. Canagarajah (1999) comments that, 
"in considering how social, economic, governmental and cultural institutions effect inequality, [the perspective that metropolitan languages are merely hegemonic, my note] perspective becomes rather too impersonal and global. What is sorely missed is the individual, the particular. It is important to find out how linguistic hegemony is experienced in the day-to-day life of the people and communities in the periphery. How does English compete for dominance with other languages in the streets, markets, homes, schools and villages or periphery communities?" (pp. 43-44).

Nativised varieties of metropolitan languages such as Indian English, South African Black English or Mozambican Portuguese are varieties borne from a different ecology partially, if not wholly, in opposition to a global metropolitan standard form. Canagarajah claims that "[n]ativized or indigenized versions of metropolitan languages, new post-colonial literatures formulated in the metropolitan language, and the substitution of vernaculars in conventional contexts previously reserved for use by the metropolitan language alone - all these are quiet ways in which resistance against excolonial languages has already begun" (1995: 593). In other words, the promotion of local systems of meaning or ways of using language on public arenas comprise the most powerful alternatives to the status quo.

Using local varieties of metropolitan languages in the classroom, both as objects of teaching and as medium, may enhance local community input in two ways. Firstly, if the teaching of a metropolitan language could capitalize on the way local voices are using the metropolitan language of power, and acknowledge diversity in the form of local knowledge structures and systems of language, this would undoubtedly promote equity and community ownership of language.

Secondly, locally generated metropolitan language practices can also be used to help minority speakers gain a critical access to more standard forms of the metropolitan language, with the understanding that non-standard forms are equally valuable and articulate other types of community and social identity. Norton-Pierce (1989), for English teaching, says that

[t]he teaching of English can be reconceptualized as a pedagogy that opens up possibilities for students and teachers of English, not only in terms of material advancement, but in terms of the way they perceive themselves, their role in society and the potential for change in society. (Norton-Pierce, 1989: 402)

This implies that an emancipatory "counter-discourse" of L2 teaching should build on the ways in which the language is used in the learners' immediate social context. Teachers may find it expedient and pupils may find it fruitful to explicitly compare and contrast, and in other ways, treat, differences between the pedagogical prescriptive norm of the classroom and the norms encountered in the learners social context. Pennycook (1994) points to one way forward in the direction of a critical language pedagogy in the following words:

...first we need to make sure that students have access to those standard forms of the language that are linked to social and economic prestige; second, we need a good understanding of the status and 
possibilities presented by different standards; third, we need to focus on those parts of the language that are significant in particular discourses; fourth, students need to be aware that those forms represent only one set of particular possibilities; and finally, students also need to be encouraged to find ways of using language that they feel are expressive of their own needs and desires. (Pennycook, 1994: 317-318).

Clearly, this view has implications for the notion of language mastery and proficiency. To master a language is not only to be proficient in a standard form, but also to have the ability to use the resources of the language to express alternative voices that may conflict with the mainstream.

Further upgrading of metropolitan language proficiency is probably needed, as many teachers themselves complain about lack of sufficient skills in the metropolitan language

Principle 7. Teacher training should be conducted in the language and culture of the community.

This principle may sound obvious, but it is far from the reality of teacher training as practiced today. Teacher training is often in the metropolitan language, as are syllabi and teachers guides. This implies that teachers will leave their training little familiar with the native language as a language of instruction, and not being comfortably biliterate. In point of fact, teachers should also be offered proficiency training in the local language, especially in the area of literacy skills, as many teachers will not have had literacy training in the language prior to having to teach it.

Training should be provided in how to teach the future language of instruction, and trainers and manuals should also use the vernacular to this purpose in order to familiarize the teacher with the appropriate terminology and register of the disciplines. Teacher trainers would benefit from teacher training texts in national languages of instruction, and one priority should be to provide adequate lexica for teachers, and, more generally, teachers' guides in local languages. This could be one task for teacher training institutes. Remedies such as this will also indirectly contribute to the sorely needed intellectualisation of indigenous minority languages.

One advantage of decentralized teacher training in indigenous languages is that the teachers' insider knowledge of what makes for a 'guiding-lights' pedagogy in indigenous language contexts can be more easily incorporated.

The fact that the teachers themselves may be the only pedagogical input that pupils in rural areas will come into contact with should have consequences for how they are trained. Teachers must receive training where, for example, materials are non-existent, and should emphasize how to attain curriculum goals on the basis of poor texts and limited materials in different school conditions. Teacher training should also experiment with different ways of pursuing local research and development on teaching of local languages. There is in fact no better training instrument that that which allows the teachers to participate in development of materials and routines.

Principle 8. Production of materials should be decentralized to the language communities as much as possible.

Materials production is an important, albeit problematic, component of 
multilingual programmes. Book production can be an important guarantee that bilingual education programmes are actually implemented. Textbooks in local languages may sometimes comprise a 'political weapon' - reducing the impact of an unfavourable, nonchalant or disinterested politics on bilingual education (Komarak, 1996). Books may provide a means for supervising teachers, determining content and progression in the absence of a curriculum, quite apart from serving as a tangible manifestation of the literate presence of African languages.

Printing costs and availability of books are two major problems for the successful provision of multilingual services. Modern information technology offers all sorts of enticing possibilities for producing literacy materials in the community themselves, such as, for example, the employment of modern techniques of desktop publishing. Another method the so-called MultiStrategy Method, (Stringer and Faraclas, 1987) which the authors claim enable "people to create relevant mother tongue literature at the local level in such a way that educational impact is high and financial impact is low". They suggest that teachers may be trained in this method to increase production. Decentralization of materials production will increase materials availability and cut production costs. In general, teachers could be trained in developing materials as the outcome of the teaching process rather than working with published materials at the outset. Teacher training institutes might also find it worthwhile to train teachers in materials production and bilingual textbook design. This could be accomplished in the form of a hands-on, action-research experience an experience that they will surely find beneficial when in "the field" - and through providing frequent and regular seminars. At the very least, teacher feedback on teaching materials should be gathered as extensively as possible, as research has shown that feedback is a crucial component in writing relevant materials.

In line with one of the overriding aims of multilingual education, that of regional peace and cooperation, materials production can also slot into the development of regional markets. There is a need for cooperative development work in terminological, register/style and lexicography over the whole of Southern African region. This suggests the development of regional cooperation and collaboration, in research and development projects. Interregional publication and marketing of books in languages where this is possible, for example, in the languages of north-western Mozambique, Malawi and Zimbabwe would be one step towards this goal. Likewise, regional co-operations and translations of local materials into larger languages such as Swahili would extend markets. This type of language development work should go hand in hand with the production of (adult) literacy materials, which would also extend the market for language materials.

Employing local authors (under supervision of linguistic consultants and Ministry textbook evaluation units) would increase the cultural relevance and authenticity of materials, and thereby also their availability to a wide selection of readership. Cultural centres for production of stories and plays in these languages would help restore community ownership of languages, and increase the spread of varieties available for literate use. There is also the need for higher centres - universities that deal with these languages, or aspects of them, 
for example, the development of subject specific registers. In this context, high school and university students could be requested to produce materials.

There is evidence that materials that are produced locally and that link into adult community networks of local and regional economic activities are more sustainable than materials that are produced only for the school. Literacy and print are major resources not only for language development but also for language preservation. Decentralization of materials production can potentially contribute to local language maintenance and restorative activities. Community participation is essential here.

Principle 10. Multilingual education needs to be integrated into other spheres of society. Appropriate legislation should be formulated and appropriate steps taken for integrating language education into economic development (see also principle 11).

Policies on language and education invariably deal explicitly only with educational matters of language. There is rarely any attempt to link questions of language in education to extracurricular issues such as employment, social welfare, or political participation. As Fishman (1991b) has pointed out, language always exists in a cultural matrix, and it is this matrix that needs to be supported rather than the language per se. From the point of view of language maintenance, there is evidence that including minority languages in schools, although a necessary condition on its acquisition, survival and use in the community, is far from a sufficient condition for the maintenance and development of a language. Attitudes towards a language are the symbolic expression of speakers' structural position in society (see below under ethics, 8.2). This means that attitudes are not easy to change without a real change in the social conditions that frame the use of language. There has to be something that makes a community want to hold onto its language(s). Parents need to feel sufficiently motivated to socialize their children in the language, and this they can only do if the language has a viable and vital role to play on important arenas of people's social life.

Experiences has shown that the development of niche economies, such as tourism or handicraft, which in turn lead to higher incomes, greater prosperity and the development of a middle class or a business sector, contribute positively to a community's determination to maintain their language(s). More fundamentally, as we have noted in chapter 8 , language programmes need to be a central part of a sustainable local programme of development.

Bernasek and Stanfield (1997) discuss how community managed micro-lending through the construction of the Grameen bank in Bangladesh has resulted in increases in vernacular literacy. Veloso (n.d.) reports on how the development of literacy skills was pursued by peasant women in rural areas of Mozambique in conjunction with agricultural cooperatives. Robinson (1996) documents how the use of local languages to manage development projects in Cameroon drastically improved not only their relevance to the local community but also their productivity. A contextually relevant educational programme is one way in which language may come to play a more important role in the community generally. However, this also means that language programmes need to de-emphasize the linguistic bias of such programmes. Decentralization of multilingual programmes would aid the integration of the school programmes in 
real, every-day multilingual networks of interaction, and thereby make language programmes more relevant to a range of community needs. This would also have the added advantage of contributing to the vitality of the indigenous languages.

How then to contextualise educational issues in relevant national legislation on language and labour rights, citizenship rights etc.? More recent approaches to the politics of linguistic representation in the age of twentieth-century pluralism, has been the notion of linguistic human rights. There are, however, a good number of problems with this concept. One of the most vexing is a general problem with a right's approach to social issues, namely its selective and potentially discriminatory and socially divisive nature. To be workable, rights discourses often tend to select disadvantaged groups for special treatments at the felt cost of the majority, such as endorsing positive discrimination on the labour market. Opposed to a rights perspective, therefore, are many who "see the ideals of democracy as pointing towards a politics in which people transcend their localized and practical concerns, getting beyond the narrow mechanism of special interest to address the needs of the community instead" (Phillips, 1995: 290). An alternative notion to linguistic human rights that attempts precisely to factor concerns with language into an ethically and socially just and general and transformative political discourse is linguistic citizenship (Stroud, ftc.). The concept of linguistic citizenship has a number of advantages in comparison to its sister notion, linguistic human rights. Firstly, it is a way of addressing the need for a new concept of citizenship that mediates between "the universal and the particular, between rational and individual expression" (Tourraine, 1994, quoted in Silverman, 1999: 147) through using avenues of commonality to enhance respect for diversity and make room for different forms of individuality (Mouffe, 1993). As such, it has the potential to both advocate and retain local perspectives on empowerment and avoid charges of political particularism. Secondly, linguistic citizenship addresses the very real materiality of language in minority politics by attending to the fact that linguistic minorities suffer from both structural and valuational discrimination. In other words, the injustices that befall speakers of minority languages are related to the structural position that minority speakers have in the politico-economic order at the same time as they are also clearly a reflex of minority speakers' identities as minority language speakers, as the social structures that minority speakers are part of create conditions of existence which are both material and discursive. Fraser (1995: 85) refers to such a collectivity as a bivalent collectivity for which neither "socioeconomic maldistribution or cultural misrecognition are an indirect effect of the other, but both are primary and cooriginal". Bivalent collectivities require concerted and equal action on both structural and ideological or valuational dimensions; issues of redistribution must be explicitly linked to linguistic identities. The notion of linguistic citizenship does precisely this - it links language, subject position and issues of redistribution by locking language as a political and social concept firmly into discourses of welfare and equity.

A third advantage of a citizen concept is that it encourages the strategic use of broad coalitions. Focusing on language issues specific to particular ethnic groups and their requirements from a rights perspective runs the risk 
of creating ethno-linguistic division. The concept of linguistic citizenship permits the formulation of political stands on language issues common to minority speakers in general. It encourages commonality of action and commonality in action, rather than a politics based in group characteristics of a more essentialist nature (Simons, 1995). Attention to what unites actors with respect to language, however, does not preclude sensitivity to issues that divide speakers of different minority languages and how they are positioned in relation to each other. Language activists do not have to agree on a unified and coherent vantage point. Should linguistic citizenship ever be seriously debated in the future, we might expect that contending political interests will offer conflicting representations of speakers and their rights, as is the case with, for example, gendered notions of citizenship (the different notions of feminism). But in like manner to how gender has been variously politicised over the years, with extensive implications for the development of gendered subjectivities and gender politics, so too might the notion of linguistic citizenship extend and diversify debates on the importance and reach of language as a socio-political category. The important point is that this approach to revitalisation also draws on the power of linguistic identity - although non-essentially defined - to mobilise minority language speakers.

Finally, linguistic citizenship can encourage a grassroots' grasp of the notion of language. Language is very much a constructed and contested object and the socio-historical outcome of debate, legislation, divergent ideologies and social conflict. Instead of language viewed solely as a formal object, a notion of language as a pragmatic and consensual set of practices brought into being by a community of users is available for exploitation. Such a view of language has the potential to avoid sculpturing indigenous languages in the image of more powerful and dominant languages, and is one way of side-stepping the frequent divisive and discriminatory debates on purity and correctness that are so detrimental to language maintenance and revitalization (see e.g. Hornberger \& King, 1998; Dorian, 1994).

Principle 11. Adult literacy for programme sustainability.

Many studies have acknowledged the important role of children's primary network of caregivers in forming their language and literacy development. Children quite simply learn to read better when parents-teachers interact, when they themselves have ample opportunities to practice reading, when they pay explicit attention to metalinguistic and metacognitive exercises or tasks that structure reading, and when they have access to a wide range of literacy materials in their environment. Related to this is that sustainable literacy development and effective MT education for children presupposes a literate adult environment, and a number of studies have shown that, in contexts where adult literacy programmes are running, school literacy in mother tongues is enhanced. How then do local conditions need to be organized in order to sustain schooled use of indigenous languages? One answer to this is to that local control over language education should have the beneficial effect of encouraging the input of parents and other caretakers' socialization strategies in the teachinglearning process.

Another way forward is to employ a range of auxiliary strategies and sources to create a literate milieu such as the 
establishment of small libraries or, more generally, local or provincial, cultural centres which may house quantities of available literate materials in local languages. Such cultural centres could be responsible for workshops where culturally competent elders could be assisted by language committees or specialists to capture traditional narratives, where local news bulletins could be produced, and plays and other cultural events staged. The major input here would be in terms of the provision of scarce resources such as paper and pens, and hand presses.

However, the successful attainment of literacy in the Cree case referred to above shows that appropriate literacy can be attained without books and other material accompaniments. In other words, an important hallmark of literacy is that it implies specific ways of dealing with information. Literacy in local languages can very well be anchored in activities of the community, as this makes literacy socially meaningful; skills of reading, writing and learning through print in a natural social environment contextualises school literacy. Encouraging local work related literacies may require adult literacy classes in basic literacy and numeracy, the compilation of records and archives.

\section{Principle 12. Good schools.}

Numerous studies inform us that if a bilingual or mother tongue programme is situated in schools where teachers know what they are doing, where the principals are good leaders and where parents are attentive to the schooling of their children, these programmes tend to be more successful than programmes located in poorer quality schools. Subsequently, it makes sense to suggest that bilingual provisions in the form of more general teacher skill variables, organization and administration of schooling etc. should also be paid serious attention. For example, teacher preparation for bilingual education should not halt at providing didactic solutions specific to bilingual contexts. Many teachers will also need radical schooling in general didactic philosophies and the provision of more adequate role models for their role as teachers. Studies show how the advantages of mother tongue schooling, such as increased pupil participation, are sometimes lost through teachers' rigid adherence to frontal pedagogical techniques. Teachers may, for example, perceive active pupil contributions to be a threat to classroom order. Teachers trained in systems of education using metropolitan languages may not realize to what extent their rote repetitive classroom techniques were a result of having to teach in a language that no pupil understood. Generally, however, teachers need to have sufficient quality in more general pedagogical aspects, as well as good subject specific knowledge that will allow them to meet the demands of teaching different subjects at different primary school levels.

A study by Freeman (reviewed by Corson, 2000) argues that an important parameter in ensuring an adequate implementation of MT teaching is that the local school can withstand the surrounding societal pressures for a language shift to the metropolitan language. Teachers who can comfortably work together in an informed way around issues of multilingualism and reach consensus and closure on appropriate teaching practices and the need to 'protect' the indigenous language speaking child from stigma and other pressures succeed better. Corson, says: "To make this different orientation [language as resource, my note] work, the Oyster school had to take itself very seriously as a single community with common interests and common goals, rather than a set of distinct communities of different ethnocultural groups often in conflict with one another" (2001: 
201). The Oyster school programme started as a grassroots initiative, and developed and incorporated many aspects of programme and practice that went against mainstream educational and societal discourses, such as a maintenance approach to language education, recruitment of culturally competent teachers, culturally sensitive negotiation of classroom discourse norms, etc. (cf. Corson, 2001).

Finally, a point relating to educational policies to promote good schools concerns the general nature of the services offered, rather than restricting the provision of bilingual education to disadvantaged groups only. Multilingual programmes should offer language provisions to a wide segment of pupils. A characteristic of successful bilingual programmes is that they have tended to cater to a broader population. Programmes that are integrated into good schools and/or are run for or by mainstream organizations or parent associations, such as the Canadian immersion programmes, tend to be more acceptable and more successful than programmes targeting minority or socially disadvantaged populations. Depending on the type of programme or curriculum solution, schools may also serve to recruit (second language (L2)) speakers of a minority language. However, Mazrui (1997) estimates that the majority of the pupils who get targeted for special bilingual education policies are workers and peasants, making bilingual education traditionally into a class issue. Clearly, there is scope here for providing bilingual educational options for majority language speakers more generally, as has been attempted with some success in Latin American contexts.

\section{Summary}

In the above, principles for educational language provisions have been framed in a socio-politically sensitive understanding of the workings of language and power. All of the proposals have quite far reaching implications for principles and practices of materials development, teacher roles and methods. They mean stepping back from purely educational solutions to problems in multilingual education, and recognizing the importance of community participation and the development of an adequate socio-cultural matrix that can sustain local literacies and languages in a 'learning for life' context. They also involve acknowledging the complex ways in which questions of politics and power impact on many different levels of multilingual education.

In the final section, we will consider some important dimensions in implementing bilingual and mother tongue programmes that often are overlooked and that may thwart the successful implementation of language education.

\section{SOME COMMON PROBLEMS IN IMPLEMENTATION \\ OF MULTILINGUAL EDUCATION}

\section{Introduction}

The thrust in the preceding has been to detail ways in which official institutions may need to be changed to cope with linguistic diversity in frameworks of sustainable development and local decision-making. However, there is invariably a range of pressing problems 
remaining, some of which arise from the nature of proposed strategies and remedies in this report. In the following sections, I list very briefly some of these problematic issues, and suggest some ways around them.

\section{Ethical issues in the provision of programmes}

Even though the primary goal should be to provide mother tongue education in as many languages as possible, there will always be more languages than there are re-sources in the form of manpower, materials and money. It is also the case that MT language provision for some minority languages is not a viable option for various reasons. Especially for those communities that have undergone extensive language shift, the rudimentary levels of linguistic competence among community members may not be at a level sufficient for educational use. Even with massive input of revitalization efforts, it might prove difficult to reach desired proficiency levels. Policies for $\mathrm{Mt}$ language provision need to be flexible and sensitive to local conditions, which even entails not categorically providing for provision where communities do not request, want it, or would benefit from it.

How then to manage the difficult and ethically contentious task of deciding in what contexts and to what extent Mt provision should be offered, and with what degree of commitment or persuasion? With regard to societies undergoing language shift in dire need of revitalization, Fishman (1991) has designed an eight-stage scale to measure the vitality of the minority language. The highest stage in the scale designates a community whose language is no longer spoken by the youngest generation. In this situation, trans-mission of the language has ceased and it is to all intents and purposes lost to the community. In such a case, any attempt to provide education in the language will require a great deal of work in reinvigorating the language, for which there may no longer even be any heartfelt need or motivation on behalf of the community itself.

Corson \& Lemay (1996) has suggested a set of action principles of an ethical nature that could be applied to these sorts of situations, whereby it should be possible to attain the maximum provision of mother tongue education possible. These authors suggest:

- The first policy principle guarantees the right of children to be educated wherever possible in the same variety of language that is learned at home or is valued most by them.

- When the first policy principle cannot be met, the second principle guarantees the right of children to attend a school that shows full respect for the language variety that is learnt at home or valued most by them, including respect for its role in preserving important ethnic, traditional, social, gender, or religious values and interests. In other words, use of the minority language variety would be encouraged and valued in every school context, even while it is not used as the vehicle of instruction.

- The third policy principle guarantees the right of children to learn, to the highest level of proficiency possible, the standard language variety of wider communication used by the society as a whole. (quoted in Corson, 2001: 32).

There are, however, other aspects involved in the provision of MT education. In chapter 5 on language shift, I noted how Tok Pisin has become essential in the social reproduction of Gapun villagers' 
cultural conception of Self. In many subtle ways, Tok Pisin has come to symbolically stand for valued and desired aspects of Self such as consensus, knowledge and social maturity that villagers admire and refer to as save. The local Taiap language, on the other hand, has become uniquely associated with traits such as femininity, tradition and children, as well as traits such as stubbornness, aggressiveness and egoism, collectively referred to under the label hed. It was traditionally the case that both save and hed were encoded in the local Taiap, but over time, these cultural values have become locked into two separate codes or languages. In other words, although villagers are still socializing their children into culturally competent community members, and the traditional conceptions of self so important to the smooth running of the society are being carefully maintained, this is done through the multilingual juxtaposition of two codes rather than one. The situation in Gapun village is a special case of the general and ubiquitous division of labour between languages, and illustrates the idea of language portfolio introduced in chapter 8 .

The ethical issue that this raises addresses the problem of cultural imposition that promotion of Taiap in Mt education would involve. It would namely be the case that (re)associating Taiap with those values that are currently carried by Tok Pisin would undoubtedly change the configuration of cultural and personal values linked into each language, and have ramifications for socialization, perceptions of power and order etc.

A further point related to all of this concerns the issue of negative attitudes on behalf of community members towards the use of local languages in formal systems of education. We are all too ready to complain that such communities might not know what is best for them, that they may be deceived in their perceptions of the usefulness of metropolitan languages, and that their attitudes need to be changed. There are even some examples in the literature where caregivers who were initially negative towards Mt education became persuaded of its benefits and ardent supporters after having had their children take part in programmes for a while. But these cases are rare. The issue is complex. Many of the remedies that the current report proposes depend on the engagement, interest and voluntarism of the local community to take issues of Mt seriously as part of a more general emancipatory discourse. Forcing caregivers to engage in Mt education goes against the whole grain of such a project, and would surely only lead to the failure of projects that did not have community support from the outset.

In recent years, languages in danger of loss or death have been accorded a great deal of professional and public attention. Many authors represent language shift/loss as a human and ecological catastrophe, with languages in need of extensive linguistic salvaging in order to turn the tide of current developments. There is also a powerful ethical issue involved in language loss/ shift. Crawford (1998) has noted that "we should care about preventing the extinction of languages because of the human costs to those most directly affected" and goes on to say that "after all, language death does not happen in privileged communities. It happens to the dispossessed and disempowered peoples who most need their cultural resources to survive." (p. 163). Coulmas (1998) says that claiming that language shift is a catastrophe might depend on a "nineteenth century romantic 
idea that pegs human dignity as well as individual and collective identity to individual languages" (p. 71). The heavily metaphorically laden discourses of language loss/shift are in fact part of larger discourses of moral pastoralism, that is, "a tradition that continually looks back, often nostalgically, and for moral guidance, to a lost but supposedly more pristine and authentic past" (Gal, 1996: 587). It is important to hold in mind that, despite the numerous organic metaphors, such as death, murder, health, decay, suicide etc. that are used to talk about language shift/ loss, the process can only really be understood in terms of the "socio-political practices, cultural understandings, discursive processes, goals, and cognitive constraints that motivate the speakers and social groups that bring about the linguistic changes" (Gal, 1996: 587). Although pastoral discourses of loss have played an important role in publicly promoting ethical issues implicated in the plight of minority languages and indigenous peoples, we also need to keep in mind that local authenticity and indigenous cultural expression can also be manifested by means of new or indigenous varieties of language. In fact, restricting authenticity to local indigenous languages, is yet one more instance of the effect of the ownership parameter in the standard language debate. By discussing authenticity and indigenousness only in terms of local languages, the important role played by these evolving varieties risks being overlooked.

\section{Cost affordable}

In the 'new world economy', decisions on the desirability, efficiency, relevance etc. of an institution, programme or activity is more and more in the hands of accountants, and the success or failure, the survival or death of an educational programme, is measured predominantly in economic terms alone. MT education has always been subjected to penetrating analysis from cost-efficiency perspectives. Generally speaking, however, there is still no way of determining exactly what MT programmes bring about in the form of added expenditure. Authors such as Dutcher (1997) claim that teaching a child in the mother tongue costs no more than teaching the child in another language, pointing out that both teacher salaries and materials need to be available in any event. Costs only rise in comparison to normal schooling when extra teachers need to be hired. However, Dutcher's reasoning is true only given the supposition that all the necessary development work has already been carried out, that is, that sociolinguistic and community attitude surveys have been conducted on appropriate languages, that languages have been described and standardized and materials written, that teachers have been trained, and evaluation measures planned and implemented. It is precisely these developments that carry the brunt of the increased expenditure often noted in conjunction with bilingual programme implementation. On the efficiency pole of the equation, there is still no clear idea on what expenditure on Mt education means in terms of benefits in relation to other parts of a school curriculum, or in terms of life chances, social mobility, harmony etc.

This report has argued that there is sufficiently clear indication that providing multilingual education may reduce a range and variety of social costs incurred by the marginalisation of communities. Furthermore, decentralisation allows language provisions to be more realistically budgeted, which will save 
costs. Smaller-scaled implementation of multilingual provisions may be more cost-effective than large-scale, national, projects. The question of maximum resource utilization (economy and manpower) needs to be approached by means of a number of alternative ways of increasing the economic margins of bilingual programmes. Ways of increasing the economic sphere of action may not just be through injecting more money, but in devising more appropriate or alternative ways of working within, or circumventing the constraints of, the budget limits. Programmes that are resourced sufficiently and/or realistically succeed better than those that are under capacitated.

The question of cost, however, cannot be seen apart from the aims of multilingual language provisions. In the literature, there are numerous suggestions for reducing the cost of producing materials. Some of these suggestions emphasize production of one book for all subjects for each year instead of books for each academic subject. Books will be durable and useable over generations of school children if children are not permitted to write in them, but required to write in special note-books. This cuts costs through cutting printing needs. Furthermore, the total costs of printing textbooks will go down if the number of subjects at primary school level for which books are required is decreased. Fewer subjects mean fewer printing costs. Part of the cost for printing textbooks of course goes to the production of teachers' manuals. Savings could also be made here if teacher manuals were less detailed or less subject specific, and made to focus more on general pedagogical poses than specific disciplinary methodologies. The production of dual language books for certain subjects, where the same content is encoded in both the metropolitan language and an indigenous language will also reduce costs overall, as the metropolitan part of the book could be used throughout the national school system, only necessitating change of print to accommodate different national languages. However, what all these suggestions share is a concern with cost of books at the expense of depth, coverage and appropriateness of materials - for both teachers and pupils. General pedagogical hints can never replace discipline specific knowledge and method training. And cutting down on the number of disciplines minority language children need study puts the minority child at an academic disadvantage compared to elite, majority, metropolitan speaking peers. Once again, recommendations such as these illustrate how different choices and priorities are made for minority and non-minority children.

\section{Decision structures}

It is important to consider within what institutional structures the administration of programme implementation should be managed on a daily basis. First of all, infrastructure and logistics must be created to manage the delivery of language provisions on an appropriate scale. Given the conditions for bilingual education in many parts of the developing sphere (language and power dynamics, sociolinguistic ecologies, policy model, nature of institutions, limitations of resources in manpower and materials, etc.), bilingual education, taken in the sense of promotion of diversity, seems, quite simply, not to be the type of activity that can be easily accommodated in national school structures and/or centralized systems of educational management and control. This is even more so today, where, as 
noted above (chapter 4) and by Corson (2001: 25) "present-day education almost everywhere in the English-speaking world is set firmly within capitalist social relations", given recent developments where public policy and the world itself are saturated with the homogenizing ideology of market relations.

In the practical implementation or delivery of language provisions for education, consideration should be given to the fact that implementation is not a mere technocratic execution of policy decisions but in point of fact a political process in itself. Swarts (1996) states that

[p]olicy implementation is a multifaceted and multi-layered process with the result that the policy itself is open to many interpretations at all levels of the educational system. This in turn results in contradictions and gaps, which can be exploited in accordance with power bases. (Swarts, 1996: 117)

Decisions made by actors or institutions charged with implementing a policy reflect political choices. The actors and agencies involved in implementing a language educational reform come from various positions of interest, and many of the national actors at this level belong to the indigenous elite who may have reached their posts through, among other ways, the good use of the symbolic capital implicit in the metropolitan language. These actors may subsequently provide pockets of resistance to the implementation of a reform.

There is a certain amount of plausibility in the argument that existing government structures such as departments of education and institutes of curriculum development should be responsible for coordinating the implementation of bilingual programmes. The advantages here are many. Firstly, maximum utilization of existing structures radically reduces the cost of promoting bilingual education through saving on not having to start up new institutions, with all their requirements in infrastructure, machines, manpower etc., instead riding piggyback on e.g. distributional networks for books, teacher salaries and placement procedures already set up and in place. This solution also permits a more rapid implementation of a programme, as all structures are already in place, and no build up time is required.

Secondly, using existing structures might ensure a better integration of bilingual education into the national school system, which in some studies at least has been quoted as a positive factor facilitating wider acceptance of bilingual education. The argument of sustainability is also a factor to reckon with in this context; clearly, it is much easier to sustain structures that are older and established than new, less stable institutions.

On the other hand, from the perspective of a decentralised provision of multilingual education proposed in this report, there are some obvious disadvantages in bilingual programme implementation using already extant national educational hardwiring. Foremost among these disadvantages is that historically in many cases a nation's whole educational system has been geared towards promoting the metropolitan language at the expense of local or national languages. One should not ignore the constraints at all levels of functioning that this historical mandate places on the introduction of novelties into a rigid system. Personnel and power dynamics between different interest groups defined in terms of personal career opportunities may work against the smooth incorporation of a bilingual programme into an old structure. The advantages that might be won in time through using existing structures will probably be offset by the practical need 
for major internal reorganization, such as the recruitment of new personnel (a time consuming process), the creation of new departments within the structure to manage the daily workings of bilingual programmes, the promotion of new section heads with unclear and competitive jurisdictions etc. The visibility of the bilingual programme will be less apparent. Furthermore, the advantage of integration into existing structures of education that such incorporation might provide, need not necessarily be all advantage as there is the risk that national programmes will swallow the bilingual programme.

One advantage of creating a new set of administrative structures for bilingual education is that these structures could be both cooperative and competitive with national structures as needs and conditions dictate. It is also easier to 'reorganize' or construct new structures on new principles, for example, with a heavy provincial bias. Furthermore, new work routines and inter-departmental collaborations are easier to initiate. For example, a department of bilingual education would need to establish links with and work closely with local and provincial departments of labour, culture etc. A new department could also recruit new people from the language groups themselves that would greatly increase the symbolic capital of the language and the value of activities related to the promotion of the language. It would also be a concrete step well in line with a pluralist programme for multilingual national development.

\section{Teacher training and job satisfaction}

One problem that a decentralized and local provision of multilingual education services will encounter is that many teachers, when upgraded, move to urban areas. Clearly, different types of strategy that may increase job satisfaction in rural areas is needed. Alternative ways of augmenting teachers' salaries and improving conditions, by means of, for example, local community and national trade union activities need to be found. This could be developed in the form of "gardens" or school crafts that would bring in more money to staff generally. Bilingual teachers associations could be formed where these do not exist in order to increase a sense of pride and professional identity. General and very basic improvements in professional conditions, such as upgrading of office space, providing coffee rooms or kitchens extending school library facilities etc. will also contribute to better well-being. Teacher's Unions need to be encouraged to follow up problems of professionalisation of bilingual personnel at national level.

Aside from these issues, there are a number of sensitive political factors that deter-mine the organization of teacher training. Where should the training of bilingual teachers take place? Within what type of structure or logistical framework should it be conducted? Generally speaking, in line with the decentralized approach to bilingual education advocated in this report, the teacher training institutes should be placed in the provinces or areas where the languages are used as instructional media. This would facilitate recruiting candidates with the desired languages, and provide a necessary "handicap" for those candidates who may like to compete for a place at a teacher training institute that come from majority language or other-language back-grounds. To ensure sufficient candidates with the 'right' languages, some form of quota system might need to be introduced. Decentralized teacher training could 
also be used to benefit more locally appropriate teacher training. Teacher training institutes should also be small in intake to maximize educational efficiency.

The question of how this training should be implemented raises a number of possibilities as well as several problems. Pre-service training of teachers has a number of advantages when it is a question of putting a new system of education into place at a national level. It is clear that in-service training of new teachers cannot meet the demand for teachers in an expanding system of bilingual education. Pre-service training, however, requires a large input of cost, and if taking place within existing institutions needs to proceed slowly in order to give the institution time to adapt. This problem of pre-service training is compounded by the fact that governments need to develop and certify and control such a training, which tends to delay the implementation of teacher training. In-service training has the advantage of using teachers already versed in classroom methodologies and the problems of teaching, but has the disadvantages that teachers' conceptions of what teaching is about are already well grounded and not always adequate. In-service training of bilingual teachers also comes up against the problem of mobility and language competencies of teachers who had been placed in schools in an old, metropolitan system. However, in-service training is hands-on training; it reaches larger numbers of teachers, and this allows for the rapid distribution of new methodologies and insights. The problem is how to distribute resources across these different services. Generally, how resources are distributed between these different services is a question of local choice based on many different local contingencies. In experimental situations, or in contexts where governments might not be enthusiastic about bilingual education, in-service training might be the preferable or most viable alternative. It could be seen as a strategy for disseminating the culture of bilingual education, and for preparing the ground for future bilingual programmes (cf. Komarek, 1996, for an extended and insightful discussion on these issues). Where governments have made decisions to implement nation wide bilingual schooling, pre-service training is an important additional component given the necessary levels of funds.

In-service training furthermore could take place also in schools at the district or provinciallevels. Onewaywould be to develop systems of pedagogical advisors for classroom observation and assistance (cf. the Mozambican system of Zones of Pedagogical Influence, which designates a grouping of schools into units under chosen pedagogical leaders who assist teachers with lesson planning and other day to day affairs of classroom management). The pedagogical advisors could be chosen from among especially competent or proficient teachers in the local schools concerned Supervision should also be coordinated with a research and evaluation unit. One option for teacher training is to use self-study or distance materials in radio instruction. However, not all teachers may have the experience or capability of working independently with such materials. Generally, a system utilizing pedagogical advisors should be built up on a local basis, allowing easy daily contacts and exchanges be-tween schools.

The length of training should also be carefully considered. Pre-service training should probably be ideally dimensioned at around 2-3 years. Likewise, time allotted to in-service training needs to be expanded considerably - two weeks every six months, for example, is far from adequate to meet the demands of a new teaching philosophy. In both cases, intensive hands-on training in context, 
that is, an apprenticeship model is to be preferred.

There should be higher educational institutions where it is possible for good teachers to go, become even better and receive certification (see above on regional centres or universities). In these establishments, there would be a clear bilingual teaching option that students could follow - perhaps to a degree or diploma level. Stipends should also be available for this. This would also have the advantage of bringing practical teaching experience with bilingual forms of teaching back into teaching institutions. It would also quite likely increase the status of and demand for bilingual education.

Staff working at central institutes also needs professional staff upgrading, for example, National Institutes of Education Development, staff. Teacher trainers should have field experience of teaching in schools. Currently, many supervisors do not have access to most recent theory and practices of bilingual education. It is especially important that the school leaders are supportive of and understanding towards bilingual education, as many studies show how a good school leadership is an important factor in the success of a programme. Likewise, all personnel should have requisite language competencies. This includes secretaries, school leaders and others. The reason for this is that an environment saturated with the language of schooling pro-motes its use in diverse and varied functions even outside of the classroom.

\section{On relevant applied language research}

In many countries, the cultivation and development of local languages is of low priority and commands few resources in national budgets. One way around reliance on government units would be to set up NGOs (like the British based literacy organization, REFLECT) to do this type of work - in close collaboration with local communities. Another requirement is the development of a more relevant, applied linguistics, training of field linguistic personnel. The fact that the bulk of descriptive work is carried out by scholars who are non-native to the community, or scholars who are trained in Western academic institutions of theoretical linguistics, is far from satisfactory. Not infrequently, the concerns and interests of Western academics are geared to career structures at first world universities, and revolve around theoretical minutiae. These priorities are not necessarily the first concerns of third world scholars in educational linguistics, who need solid descriptive studies of languages that can be used for applied linguistic purposes. Obviously, training as an academic linguist is an asset, although it needs to be subordinated more relevant concerns. One promising attempt to address this problem was the recently toppled LICCA network - an organization comprising both African and European researchers who were committed to developing alternative and more relevant work on local African languages.

Of prime importance for an approach to revitalisation that empowers speakers of the languages concerned is the development of an applied linguistics discourse that is a joint construction of expert and speakers of the languages. I have also suggested that we should explore what institutional framing conditions the development of such an AL discourse requires. Many more intricate questions remain to be discussed, and have not even been broached in this report. Future studies might address the politics of the consumption, production 
and circulation of ideas on language - the political economy of language, to use Irvine's terminology (Irvine, 1986). It would surely be beneficial and necessary for the language politics I have attempted to sketch here to chart the role of 'public intellectuals' in African public discourse (cf. also Warren, 1996). Are there public intellectuals? What is the relationship between political economy and intellectual agendas? How do public intellectuals refute and dispute power structures? How do social critics see the application of their thinking to wider social movements? What involvement do intellectuals typically have in a community? What is the ensemble of social relations that producers of authoritative knowledge partake in? Answers to questions such as these would definitely aid us in designing reasonable and working strategies for doing community-based language work of relevance to issues of revitalization.

What needs to be isolated is what characterizes the diverse settings where people find voice as social critiques and producers of knowledge? Generally speaking, concerns such as those noted above are more likely to be addressed in political contexts that allow the formation of alternative and parallel institutions of AL. This might come about in the wake of a new government in concert with other tangible social and structural changes. In South Africa, for example, the formation of the University of Cape Town based Centre for Applied Language Studies and Language Services in Africa (CALSSA) and the institutionalisation of the Project for Alternative Education in South Africa (PRAESA) have offered historical opportunities to address a broad range of grassroot language concerns, previously ignored. Young (ms) underscores the felt need to reconceptualise applied linguistics in South Africa in the aftermath of Apartheid. In Malawi, new post-Banda politics encouraged the formation of new language research units, which are capable of addressing the many issues raised by the country's multilingualism. Tanzania is another case in point, where a socially concerned Ujama linguistics took off after independence.

The principle question we need to answer is under what circumstances can social critique and knowledge production become institutionalised as an autonomous discipline? Bordieu's notion of field autonomy pertains to the extent to which the practitioners in the field ${ }^{2}$ are in control of deciding on and distributing individual and collective 'rewards' or capital as opposed to outside agents. A number of factors typically delimit whether a field can be considered autonomous or not, one of which refers to whether there exists a body of independent theory, a canon of research, and a set of research procedures that uniquely define the discipline. Related to this is the existence of so-called consecrational institutions such as critics, refereed journals, associations for applied linguistics (such as AILA, the International Association of Applied Linguistics), and the ability to translate issues from outside the field into field relevant concepts. Other important factors comprise to what extent the field generates its 'own capital' and whether its practitioners partake in a reverse economy, that is, are motivated to work within applied linguistics for the discipline specific rewards, such as academic prestige, that are shared among successful practitioners. Field autonomy for a specific discipline of AL is important simply because the way a discipline is institutionalised dictates how power is manifested and by whom, for example, who gets to decide syllabi, 
recruitment of students and direct and manage research proposals, type of academic status conferred on different representatives of different academic discourses etc.

One factor that determines the social composition of the field and the type of research undertaken, and thereby indirectly the way a discipline becomes institutionalised is funding. From the British context, Brumfit (1985: 71-72) notes how funding structures in interaction with the age profiles of those who study and go on to practice AL severely constrain the type of work that can be done. Brumfit argues that applied linguistics research conducted in Britain is characterized foremost by a generalist stance, the application of theories from multi-disciplines, and the declaration of independence from theoretical linguistics, as opposed to immersion in data bases specific to a problem area. He explains this precisely as a direct consequence of the level and type of funding available to researchers. Closely connected to the institutional and funding parameters is the training that applied linguists receive. Different types of $\mathrm{Ph}$. D. programme attract different types of student, and this favours different types of research. In Africa, if current trends continue, it will probably remain the case that the largest supply of doctoral applicants will be people working in education. These individuals are typically somewhat older than the typical post-graduate student, with a number of years' practical teaching experience behind them and with some very focused questions on language teaching, materials development and curriculum design at the forefront of their interests. They are also quite likely to be firmly entrenched in a non-academic career structure. Given the practical needs of these graduates, and the constraints in resources and classroom structures that typify their everyday working environment, it is highly un-likely that they will want to explore an alternative and grassroots approach to AL discourses.

The transnational nature of the linguistic market also exerts a strong influence on what trajectory the discipline of AL can take in African contexts. Reports of staff being sent to American and European universities to do degrees in a specific field of applied interests, and returning as fully fledged theoretical linguists are legio. African linguists are often quite simply treated as a pool for 1st World linguistics. The training they return with comprises facility with decontextualised linguistic and analytical skills, and models of argumentation and evidence that cannot accommodate plural authorities or multiple authorships. It is doubtful if linguists trained in these forms of language theory will easily embrace approaches to language that, for example, incorporate moral, political and local social concerns into research programmes, or be applicable or relevant to the social and political context of languages of the African community.

A final point related to this is the genesis of the field of AL in specific contexts. Generally, fields may arise out of other fields, such as the field of psychology, which was originally part of pedagogy, or be generated from practical concerns (see Broady, 1998). Departments of AL appear to have different origins and

\footnotetext{
2 Every field has its own specific type of capital, the symbolic or material benefits of the field - and, in fact, the notion of field was originally conceived as one means of exploring how different forms of 'capital' are forged and distributed.
} 
multiple historical sources in different polities. For example, in South Africa, many AL departments have arisen from concerns with teaching English as a Second Language (ESL). In Zimbabwe, a new centre for applied language studies has grown out of a single project on computerized lexicography. Obviously, the focus of a department will depend very much on its history and on the contacts members have with other disciplines.

All in all, current trends in financing, transnational influences, student profiles, and forms of institutional cooperation do not seem to be particularly favourable to the development of alternative and empowering AL discourses.

\section{Global discourses of multilingualism}

One danger that may reduce the benefits of a multilingual education policy is that many postmodern discourses, while superficially seeming to promote diversity and grassroots influence, may, in actual fact, serve to appropriate these polices in the service of global developments. Corson (2001) says that "one of the main features of postmodernity is a trend away from centralization and toward diversity and devolution of control [...] Yet, in hearing these new voices now appearing at local levels, powerful forces beyond the local are still ignoring the messages they raise. This is especially true of education, and it is true of education for diversity in particular" (p. 25). Gee (e.g., 2000) has noted how seemingly postmodernist radical discourses of social constructivism, and non-hierarchical work relations have been appropriated in the service of global capitalism. In like manner, discourses of multilingualism, previously the purview of pluralist and liberal political discourse, are also well on their way to becoming the new ideological tools of world market capitalism. The cultivation of a critical awareness, and a continual vigilance that the benefactors of multilingual education are the marginalized community itself, should offset the more dire consequences. However, one ought not to look askance at all post-industrial developments, as the focus on productive diversity in relation to language in a world economy will in some cases have positive effects on local languages.

\section{Conclusion}

In this report, I have been particularly concerned to critically review some of the more prominent paradigms and policies relevant to language and education in multilingual developing contexts. I have tried to argue that much of what is done in the name of a multilingual education for diversity is geared to the management of multilingualism in a world economy increasingly dominated by global multinational economies and managed by the political thinking of an international elite political community. I have consistently tried to interpret problems of language and education in a framework of power, ideology and a politics of global, mainstream, thinking, versus a framing of language issues in local discourses of sustainable development and grass-roots empowerment. In this spirit, I have argued that the principal problem confronting successful provision of multilingual education is political. I have pointed to how the abysmal condition of many bilingual programmes could be traced to the more or less complete absence of indigenous and local participation in areas of curriculum design, materials development etc. In other words, metropolitan values and realities were given priority, and this must change if we seriously wish to see 
empowerment of minority languages. In order to succeed, policy makers need to recognize the disempowering effects of their current policies (even when these policies are addressed ostentatiously to the preservation and cultivation of indigenous languages and multilingualism — such as multiculturalism), and they must seek to develop a set of political premises that can accommodate linguistic and cultural diversity instead of stifling it. The way forward is given through an examination of how multiple languages contribute to individual and social empowerment in diverse ways in local communities, and to use this as a basis for formulating new political discourses of empowerment that can underlie policy decisions. The model, in other words, is to demand public and political recognition for the lived realities and contingencies of marginalized populations by tying language issues into a politics of recognition and redistribution. The effectiveness of different forms of bilingual education depends on the distribution of power and economy in society and, ultimately issues of democracy, equity and people's political participation (see e.g. Stroud, ftc, on linguistic citizenship; Hyltenstam \& Stroud, 1996; Pütz, 1997).

To meet these goals, some radical departures from prevailing practices of bilingual programme management must be made. First of all, the distribution of power in determining the content, structure and implementation of multilingual education must be radically changed. The communities concerned themselves must be able to compose new acceptable discourses on language. This implies a greater emphasis on local and decentralized control over issues of multilingualism in education.

Secondly, indigenous languages need to be ideologically reinstated as sufficient and adequate primary languages of instruction at all levels of education. This implies that the weight that teaching in metropolitan languages has been granted up until today must be diminished, and that secondary discourses in local languages must be developed so as to facilitate knowledge transmission throughout the entire school system. Just as importantly, it involves relinquishing the idea of transitional/maintenance programmes. These programme types have long been the preferred solution to introducing mother tongues in school contexts, but they are typically a product of hegemonic thinking.

A third point that needs to change is the reigning negative appreciation of multilingualism. Schools should be willing to use more than one language in the classroom, and be prepared to adapt their teaching methodologies accordingly. The important role that mastery and use of multiple languages plays in the ecologies of minority communities should be educationally acknowledged.

And fourthly, mindset and general orientation to bilingual education needs changing. The elites of society know how to harness capital returns from multilingualism, and they design bilingual education accordingly. In Canadian immersion programmes or in Finnish bilingual schools, minority languages are being used throughout pupils' school career. In elite European Union schools, many languages, not just one or two, are used concurrently in the classroom and as instructional media. In other words, many of the principles or design features suggested in this report are, in point of fact, already being successfully implemented in other, first world, contexts. However, for some reason, the solutions that the elites themselves prefer are not considered viable in developing contexts. 


\section{BIBLIOGRAPHY}

Akinnaso, F. N. Toward the development of a multilingual language policy in Nigeria. Applied Linguistics 12(1): 29-61.

Andersen, R. (ed.). 1984. Second languages: A cross-linguistic perspective. Rowley: Mass. Newbury House.

Althusser, L. 1971. Lenin, philosophy and other essays. London: New Left Books.

Arnold, Thierry. 1989. Le Multilinguisme facteur de développement ou le paradox francophone en Afrique. In Chaudenson, R. \& D. de Roubilliard (eds.), 115-131.

Arthur, J. 1994. Talking like teachers: Teacher and pupil discourse in Botswana primary classrooms. Language, Culture, and Curriculum 7: 29-40.

Arthur, J. 2001. Codeswitching and collusion: classroom interaction in Botswana Primary Schools. In M. Heller and M. Martin-Jones (eds.), Voices of Authority. Education and Linguistic Difference. London: Ablex Publishing.

$\mathrm{Au}, \mathrm{K} .1980$. Participation structures in a reading lesson with Hawaiian children: Analysis of a culturally appropriate instructional event. Anthropology and Education Quarterly, 11(2): 91-115.

Auer, Peter. 1998. (ed.). Code-Switching in Conversation. Language, interaction and identity. London and New York: Routledge.

Bain, B. 1974. Bilingualism and cognition: toward a general theory. In S. Carey (ed.).

Bain, B. \& Yu, A. 1980. Cognitive consequences of raising children bilingually: One parent, one language. Canadian Journal of Psychology, 34: 304-313.

Baker, C. 1990. The effectiveness of bilingual education. Journal of Multilingual and Multicultural Development 11(4): 269-277

Baker, C. 1996. Foundations of bilingual education and bilingualism. Clevedon: Multilingual Matters.

Bamgbose, Ayo. 1991. Language and the Nation: The language question in Sub-Saharan Africa. Edinburgh: Edinburgh University Press.

Barnett, T. 1997. States of the State and Third Worlds. In Golding, P. and Harris, P. (eds.).

Bassnet, Susan \& Trivedi, Harish. 1999. (eds.). Post-Colonial Translation: Theory and Practice. London: Routledge.

Bedi, A. S. \& Gaston, N. 1999. Using variation in schooling ability to estimate schooling returns for Honduras. Economics of Education Review 18: 107-16.

Ben Zeev, S. 1977. The influence of bilingualism on cognitive development and cognitive strategy. Child Development, 48: 1009-48.

Bernasek, A. \& Stanfield, J. R. 1997. The Grameen Bank as progressive institutional adjustment. Journal of Economic Issues 31: 359-66.

Blommaert, J. 1996. Language planning as a discourse on language and society: The linguistic ideology of a scholarly tradition. In Language Problems and Language Planning, 20, 3: 199-222.

Blommaert, J. (ed.), 1999. Language Ideological Debates. Berlin and London: Mouton de Gruyter

Broady, D. (ed.) 1998. Kulturens fall. En antologi. Stockholm: Daidalos AB.

Brock-Utne, B. 2000. Whose education for all? Recolonization of the African mind. London: Taylor \& Francis Publishers.

Brumfit, C. 1997. How Applied Linguistics is the same as any other science. International Journal of Applied Linguistics 7(1): 86-94.

Bruthiaux, P. 2000. Supping with the dismal scientists: Practical interdisciplinarity in language education and development economics. Journal of Multilingual and Multicultural Matters, 21, 4:269-291.

Burnaby, B. \& T. Ricento (eds.). 1998. Language and politics in the United States and Canada.Myths and Realities. Mahwah, N. J.: Lawrence Erlbaum.

Cameron, D. 1990. Demythologizing sociolinguistics: why language does not 
reflect society. J. E. Joseph \& T. J. Taylor (eds.).

Canagarajah, A. S. 1995. Review of Robert Phillipson: Linguistic Imperialism. In Language and Society, 4 (4), 590-94.

Canagarajah, A. S. 1999. Resisting linguistic imperialism in English teaching. Oxford: OUP

Carey, S. (ed.). 1974. Bilingualism, Biculturalism and education. Edmonton: University of Alberta Press.

Castells, M. 1989. The informational city: information technology, economic restructuring and the urban-regional process. Oxford: Blackwell.

Castells, M. 1996. The Information Age: Economy, society and culture. Vol. 1: The rise of the network society. Oxford: Blackwell.

Cerrón-Palomino. 1989. Language policy in Peru: a historical overview. In International Journal of Sociology of Language, 77: pp. 11-35.

Chambers, Robert. 1993. Challenging the Professions: Frontiers for Rural Development. London: Intermediate Technology Publications.

Chambers, J. K. 1994. Sociolinguistic theory. Oxford: Blackwell.

Chaudenson, Robert \& Didier de Roubilliard. 1989. (eds.). Langues, économie et développement (tome 1). Aix-enProvence: Institut d'études Créoles et Francophones.

Collier, V. P. 1987. Age and rate of acquisition of second language for academic purposes. TESOL Quarterly, 21, 617-641.

Collier, V. P. \& Thomas, W. P. 1988. Acquisition of cognitive-academic second language proficiency: A six-year study. Paper presented at AERA, New Orleans.

Cooper, R. L. 1989. Language planning and social change. Cambridge: CUP.

Cope, B. \& Kalantzis, M. (eds.) 2000. Multiliteracies: literacy, learning and design of social futures. London and New York: Routledge.

Corson, D. 1993. Language, minority education and gender. Linking social justice and power. Clevedon: Multilingual Matters.
Corson, D. 2000. Language diversity and education. New Jersey and London: Lawrence Erlbaum Publishers

Cordon, D. \& Lemay, S. 1996. Social justice and language policy in education: The Canadian research. Toronto, ON: OISE, University of Toronto Press.

Crago, M. 1992. Communicative interaction and second language acquisition: An Inuit example. TESOL Quarterly, 26(3):487-507.

Crawhall, N. 1998. Going to a better life: perspectives on the future of language in education for San and Khoe South Africans. In L. Limage (ed.). Pp. 231-249.

Crawford, J. 1989. Bilingual education: History, politics, theory and practice. Trenton, NJ: Crane.

Cummins, J. 1978. Educational implications of mother tongue maintenance in minority language groups. The Canadian Modern Language Review, 34: 395-416.

Cummins, J. 1996. Negotiating Identities: education for empowerment in a diverse society. OISE.

Cummins, J. \& M. Galustan 1974. Some effects of bilingualism on cognitive functioning. Ms University of Alberta, Edmonton.

De Avila, E. 1987. Bilingualism, cognitive function and language minority group membership. In P. Homel, M. Palij \& D. Aronson (eds.).

De Beaugrande, R. 1997. Society, education, linguistics and language: inclusion and exclusion in theory and practice. Linguistics and Education 9: 99-158.

Delors, J. 1996. Learning: The treasure within. Report to UNESCO of the International Commission on Education for the 21st Century. Paris: UNESCO.

Djité, P. G. 1993. Language and development in Africa. International Journal of the Sociology of Language, 100/101: 149-166.

Dorian, N. (ed.). 1989. Language obsolescence: studies in language contraction and death. New York: CUP. 
Dorian, N. 1994. Purism vs. compromise in language revitalization and language revival. Language in society 23: 479-494.

Durgunoglu, A. Y. \& L. Verhoeven, (eds.). 1998. Literacy Development in a Multilingual Context. Cross-Cultural Perspectives. London: Lawrence Erlbaum Associates.

Dutcher, N. The use of first and second languages in education: A review of educational experience.. World Bank, East Asia and the Pacific Region, country department III. Washington, D.C.

Edelsky, C. 1991. With literacy and justice for all: Rethinking the social in education and language. London: Falmer Press.

Ellis, R. 1994. The Study of Second Language Acquisition. Oxford: Oxford University Press.

Fabian, J. 1986. Language and colonial power. Berkeley: University of California Press.

Fardon, R. and Furniss, G. 1994.

Introduction: Frontiers and boundaries - African languages as political environment. In Fardon, R. \& Furniss, G. (eds.).

Fardon, R, and Furniss, G. 1994. (eds.). African Languages, Development and the State. London: Routledge.

Fettes, M. 1998. "Life on the edge": Canada's Aboriginal languages under official bilingualism. In B. Burnaby \& T. Ricento (eds.).

Fishman, J. 1985. Language maintenance and ethnicity. In J. Fishman, M. Gertner, E. G. Lowry \& W. G. Milan (eds.).

Fishman, J. 1991(a). An interpolity perspective on the relationship between linguistic heterogeneity, civil strife and per capita gross national product. International Journal of Applied Linguistics, $1: 5-18$.

Fishman, J. 1991 (b). Reversing language shift: theoretical and empirical foundations of assistance to threatened languages. Clevedon: Multilingual Matters.

Fishman, J. A. 1994. Critiques of language planning: a minority languages perspective. Journal of Multilingual and Multicultural Development, 15, 91-9
Fishman, J., M. Gertner, E. G. Lowry \& W. G. Milan (eds.) 1985. The rise and fall of ethnic revival. Berlin: Mouton.

Fishman, J. \& Solano, F. R. 1989. Cross-polity linguistic homogeneity and per capita gross national product: an empirical exploration.. Language Problems and Language Planning 13: 103-18.

Fraser, Nancy. 1995. From redistribution to recognition? Dilemmas of justice in a 'post-socialist' age. In New Left Review, 212 (July/August), pp. 68-91.

Funkhouser, E. 1999. Cyclical economic conditions and school attendance in Costa Rica. Economics of Education Review 18:31-50

Gal, S. 1979. Language shift: Social determinants of linguistic change in bilingual Austria. New York: Academic Press.

Gal, S. 1996. Language shift. In H. Goeble, P. Nelde, A. Stary, and W. Wolck (eds.). Pp. 586-593.

Garcia, O. (ed.) 1991. Bilingual education: Festschrift in honor of Joshua A. Fishman. Amsterdam: John Benjamins.

Gee, P. 1990. Social linguistics and literacies: ideologies in discourse. London: Falmer.

Gee, P. 2000. New people in new worlds: networks, the new capitalism and schools. In B. Cope \& M. Kalantzis (eds.). Gilmore, P. \& A. Glatthorn (eds.). 1980. Ethnography and education: Children in and out of school. Philadelphia: University of Pennsylvania Press.

Gilroy, P. 1987. There Ain't No Black in the Union Jack. London: Hutchinson.

Goeble, H., P. Nelde, A. Stary, and W. Wolck (eds.). 1996. Contact Linguistics. Berlin: Mouton de Gruyter.

Golding, P. and Harris, P. 1997. (eds.). Beyond Cultural Imperialism: Globalization, Communication and the New International Order. London. Sage.

Gonzalez, A. 1993. An overview of language and development. Journal of Multilingual and Multicultural Development, 14 (1,2): 5-23.

Grin, F. 1993. European economic integration and the fate of lesser-used 
languages. Language Problems and Language Planning, 17, 2:101-116.

Hamelink, Cees. j. 1997. (ed.). Ethics and Development. On making moral choices in development co-operation. Amsterdam: kok kampen.

Heath, S. B. 1983. Ways with words. Cambridge: CUP

Hettne, B. 1987. Etniska konflikter och internationella relationer. Rapport 6 från DEIFO, Stockholm: DEIFO.

Heugh, K. 1995. Disabling and enabling: implications of language policy trends in South Africa. In R. Mesthrie (ed.).

Hidalgo, M. 1994. Bilingual education, nationalism and ethnicity in Mexico. In Language Problems and Language Planning. 18 (3); 185-208.

Hill, J. 1985. The grammar of consciousness and the consciousness of grammar. American Ethnologist, 12: 725-737

Hill, J. \& K. Hill. 1986. Speaking Mexicano: Dynamics of a syncretic language in Central Mexico. Tucson: University of Arizona Press.

Hill, J. 1993. Structure and practice in language shift. In K. Hyltenstam \&. A. Viberg (eds.).

Ho M-L. \& Platt, J. 1993. Dynamics of a Contact Continuum. Singaporean English. Oxford: Oxford University Press.

Homel, P., M. Palij and D. Aronson (eds.). 1987. Childhood bilingualism: Aspects of linguistics, cognitive and social development. Hillsdale, NJ: Lawrence Erlbaum Associates.

Hornberger, N. 1991. Extending enrichment bilingual education: revisiting typologies and redirecting policy. In O. Garcia (ed.).

Hornberger, N. \& King, K. 1998. Authenticity andunification in Quichua language planning. Language, Culture and Curriculum, 11(3): 390-410.

Hurst, P. Review of Verspoor, A.: Pathways to change - Improving the quality of education in developing countries. Comparative Education Review, 35, 3, 1991 ,

Hyltenstam, Kenneth \& Stroud, Christopher. 1993. Final report and recommendations from the Evaluation of teaching materials fro lower primary education in Mozambique. II. Language Issues. Maputo: INDE.

Hyltenstam, K. \& A. Viberg (eds.). 1993. Progression and regression in language. Sociocultural, neuropsychological and linguistic perspectives.. Cambridge: CUP.

Hyltenstam, K. \& C. Stroud 1996. Language Maintenance. In H. Goeble, P. Nelde, A. Stary, and W. Wolck (eds.). Pp. 567-578.

Ianco-Worral, A. D. 1972. Bilingualism and cognitive development. Child Development 43: 1390-1400.

Irvine, J. 1989. When talk isn't cheap: language and political economy. In American Ethnologist, 16: 248-267.

Jaffe, A. 1999. Ideologies in action: Language politics on Corsica. Berlin: Mouton de Gruyter.

Jernudd, B. \& M. J. Shapiro (eds.). 1989. The politics of language purism. Berlin: Mouton de Gruyter.

Jernudd, B. \& das Gupta, J. 1971. Towards a theory of language planning. In J. Rubin \& B. Jernudd (eds.).

Joseph, J. \& Taylor, T. (eds.). 1990. Ideologies of language. London: Routledge

Kachru, B. (ed.). 1992. The Other tongue: English across cultures. UrbanaChampagne Illinois: University of Illinois Press.

Kaplan, R. \& Baldauf jr. R. B. 1997. Language Planning: from practice to theory. Clevedon: Multilingual Matters.

Khubchandani, L. 1974. Fluidity in mother tongue identity. In A. Verdoodt (ed.).

King, K. 1999. Inspecting the unexpected: Language status and corpus shifts as aspects of Quichua language revitalization in Saraguro, Ecuador. Language Problems and Language Planning, 22, 2: 109-132.

Kokala, H. 1995. Education pays off. Helsinki: Department for International Development Cooperation, Ministry for Foreign Affairs of Finland.

Komarek, K. 1996. Mother-tongue in SubSaharan countries. Conceptual and strategic considerations for the promotion of mothertongue education in Africa. Hamburg: GTZ. 
Krauss, M. 1992. The world's languages in crisis. Language 68: 4-10.

Kulick, D. 1992. Language shift and cultural reproduction: Socialization, Self and syncretism in a Papua New Guinean village. Cambridge: CUP

Kulick, Don. 1998. Travesti. Sex, gender and culture among Brazilian transgendered prostitutes. Chicago: University of Chicago Press.

Kulick, D. \& Stroud, C. 1993. Conceptions and uses of literacy in a Papua New Guinean village. In B. Street (ed.). Pp. $30-61$.

La Belle, T. 2000. The changing nature of non-formal education in Latin America. Comparative Education 36: 21-36.

Lankshear, C. 1997. Changing literacies. Buckingham, Philadelphia: Open University Press.

Larsen-Freeman, D. \& Long, M. H. 1991. An Introduction to Second Language Acquisition Research. London: Longman.

Le Page, R. B. \& Tabouret-Keller, A. 1985. Acts of identity: Creole-based approaches to language and ethnicity. Cambridge: CUP.

Legère, K. (ed.) forthcoming. Language and Democracy. Windhoek: Longman.

Lieberson, S. 1980. Procedures for improving sociolinguistic surveys of language maintenance and language shift. International Journal of the Sociology of Language, 25, 11-27.

Limage, L. (ed.) 1998. Comparative perspectives on language and literacy. Selected papers from the work of The Language and Literacy Commission of the 10th World Congress of Comparative Education Societies, Cape Town, 1998. Dakar: UNESCO.

Lippi-Green, R. 1997. English with an accent: Language, ideology and discrimination in the United States. London: Routledge.

Liu, Z. 1998. Earnings, education and economic reforms in urban China. Economic Development and Cultural Change 46: 697-725.

Long, M. 1993. Second language acquisition as a function of age: research findings and methodological issues. In K. Hyltenstam \& A. Viberg (eds.).

Marshall, J. 1993. Literacy, Power and Democracy in Mozambique. The Governance of Learning from Colonization to the Present. Colorado: Westview Press.

Martin-Jones, Marilyn. \& Bhatt, Arvind. 1998. Literacies in the lives of young Gujarati speakers in Leicester. In Durgunoglu, A. Y. \& L. Verhoeven, (eds.).

Mazrui, Alamin 1997. The World Bank, the language question and the future of African education. In Race E Class, 38 (3).

McLaughlin, B. 1986. Multilingual education: Theory east and west. In B. Spolsky (ed.).

Mertz, E. 1989. Sociolinguistic creativity: Cape Breton Gaelic's linguistic tip. In N. Dorian (ed.). Mesthrie, R. (ed.). 1995. Language and social history: Studies in South African sociolinguistics. Cape Town: Oxford.

Milk, R. D. 1986. A cross-national comparison of sociolinguistic contexts for bilingual education. Journal of Multilingual and Multicultural Development 7 (6): 451-463.

Mohanty, A. K. 1994. Bilingualism in a multilingual society: Psychological and pedagogical implications. Mysore: Central Institute of Indian Languages.

Mouffe, C. 1993. Liberal socialism and pluralism. Which citizenship? In J. Squires (ed.). Pp. 69-84.

Muysken, P. 1984. The Spanish that Quechua speakers learn: Second language acquisition as norm-governed behaviour. In R. Andersen (ed.).

Mühlhäusler, P. 1996. Linguistic ecology: Language change and linguistic imperialism in the Pacific region. London and $\mathrm{New}$ York: Routledge.

Nettle, D. \& S. Romaine 2000. Vanishing voices: the extinction of the world's languages. Oxford and New York: OUP.

Norton-Pierce, B. 1989. Toward a pedagogy of possibility in the teaching of English internationally: People's English in South Africa. TESOL Quarterly, 23: 401-420. 
Norton, B. 1997. Language, identity and the ownership of English. TESOL Quarterly, 31(3): 409-429

Ochs, E. 1988. Culture and language development: Language acquisition and language socialization in a Samoan village. Cambridge: CUP.

Ochs, E. and Schieffelin, B. B. 1983. Acquiring conversational competence. New York: Cambridge University Press.

Othman, S. 1996. (unpubl.). Medium of instruction or medium of power? Language practices in Tanzanian education. Paper presented at International Seminar on Language and Education, Cape Town.

Padilla, A., H. Fairchild \& C. Valdez (eds.). 1990. Bilingual Education: Issues and strategies. London: Sage.

Peal, E. \& Lambert, W. E. 1962. The relation of bilingualism to intelligence. Psychological monographs, 76, 546:1-23

Pennycook, Alastair. 1994. The cultural politics of English as an international language. London: Longman.

Pennycook, Alastair. 1998. English and the discourses of colonialism. London: Routledge.

Phillips, A. 1995. Democracy and difference: Some problems for feminist theory. In

W. Kymlicka (ed.), The rights of minority cultures. (Pp. 288-302) Oxford: OUP.

Phillipson, R. \& T. Skutnabb-Kangas (eds.) 1995. Linguistic human rights: overcoming linguistic discrimination. Berlin: Mouton de Gruyter.

Platt, J. 178. The concept of a "Creoloid". Exemplification: Basilectal Singaporean English. Papers in Pidgin and Creole Studies. Series A, 54:53-65. Canberra: ANU Press.

Phillipson, Robert. 1992. Linguistic Imperialism. Oxford: Oxford University Press.

Psacharopoulos, G. Why educational reforms fail? A comparative analysis. International Review of Education. 35, 21989.

Pratt, M. L. 1992. Imperial Eyes: Travel Writing and Transculturation. London \& New York: Routledge.
Pütz, M. (ed.). 1995. Discrimination through language in Africa? Perspectives from the Namibian experience.. Berlin and New York: Mouton de Gruyter.

Rampton, Ben. 1995. Crossing. Language and Ethnicity among Adolescents. Harlow: Longman.

Rampton, B. 1997. Retuning in applied linguistics. International Journal of Applied Linguistics, 7, 1: 3-25.

Rampton, Ben. 1998. Language crossing and the redefinition of reality. In Auer, P. (ed). Rassool, N. 1999. Literacy for sustainable development in the age of information. Clevedon: Multilingual Matters.

Rehbein, J. 1984. Diskurs und Verstehen: Zur Role der Muttersprache bei der Textarbeitung in der Zweitsprache. University of Hamburg.

Roberts, C. 1997. There is nothing so practical as some good theories. International Journal of Applied Linguistics, 7,1: 66-78.

Robinson, Clinton, D. W. 1996. Language Use in Rural Development: An African Perspective. Berlin: Mouton de Gruyter.

Rubin, J. \& B. Jernudd (eds.). 1971. Can language be planned? Honolulu: University Press of Hawaii.

Schieffelin, B. B. 1990. The Give and Take of Everyday Life. Language Socialization of Kaluli Children. Cambridge: Cambridge University Press.

Schieffelin, B. \& E. Ochs, (eds.). 1986. Language socialization across cultures. Cambridge: CUP.

Schieffelin, B., K. Woolard, \& P. Kroskrity (eds.). 2000. Language ideologies. Practice and theory. New York: Oxford: OUP.

Shapiro, M. 1989. A political approach to language purism. In B. Jernudd and M. Shapiro (eds.).

Shultz, J., Florio, S, \& Eriksen, I. 1980. Where's the floor? Aspects of the cultural organization of social relationships in communication at home and at school. In P. Gilmore \& A. Glatthorn (eds.).

Silverman, M. 1999. Facing postmodernity: contemporary French thought on culture and society. London: Routledge. 
Simberg, K. 1997. Bilingual Education in Developing Countries. IV. A Bibliography and Resource List. Stockholm University, Centre for Research on Bilingualism.

Simon, Sherry. 1999. Translating and interlingual creation in the contact zone: border writing in Quebec. In S. Bassnet \& H. Trivedi (eds.).

Simons, J. 1995. Foucault and the political. London: Routledge.

Snow, C. 1990. Rationales for native language instruction. Evidence from research. In A. Padilla, H. Fairchild and C. Valdez (eds.)

Skutnabb-Kangas, T. \& Phillipson, R. 1997. Linguistic human rights and development. In Hamelink, C. J. (ed.).

Spolsky, B. (ed.). 1986. Language and education in multilingual settings. Clevedon: Multilingual Matters.

Squires, J. (ed.). 1993. Principled positions, postmodernism and the rediscovery of value. London: Lawrence and Wishart.

Sreberny-Mohammadi, Annabelle. 1997. The many cultural faces of imperialism. In Golding, P. and Harris, P. (eds.).

Sridhar, K. \& S. Sridhar 1992. Bridging the paradigm gap: Second language acquisition theory and indigenised varieties of English. In B. Kachru, (ed.).

Street, B. 1984. Literacy in theory and practice. Cambridge: CUP.

Street, B. (ed.), 1993. Cross-cultural approaches to literacy. Cambridge: CUP.

Stroud, C. 1994. Literacies. Sociolinguistic and ethnographic perspectives on reading and writing. In I. Ahlgren \& K. Hyltenstam (eds.), Bilingualism in Deaf Education. Hamburg: Signum. 201-218.

Stroud, C. 1997. Bilingual Education in Developing Countries. I. Concepts, Models and Theories.

Stockholm University, Centre for Research on Bilingualism.

Stroud, C. 1999. Portuguese as ideology and politics in Mozambique: semiotic (re)constructions of a postcolony. In J. Blommaert (ed.). Pp. 342-380.
Stroud, C., Obondo, M, Quick, B. and Thornell, C. 1997. Bilingual Education in Developing Countries. III. Dataset of Programmes, Policies and Practicies. Stockholm University, Centre for Research on Bilingualism.

Stroud C. and M. Wingstedt 1989. Språklig chauvinism. Invandrare och Minoriteter, 5: $5-8$.

Stroud, C. forthcoming(a). Language and Democracy. The notion of linguistic citizenship and mother tongue programmes. In K. Legère (ed.).

Stroud, C. forthcoming (b) Two foci for a politics of African mother tongue teaching: Linguistic citizenship versus linguistic human rights. Journal of Multilingual and Multicultural Development.

Stubbs, M. Text and corpus analysis. Computer assisted studies of language and culture. Oxford: Blackwell.

Swarts, P. 1996. (unpubl.). Language equality as a policy contradiction. The case of the Namibian language policy for schools. Paper presented at International Seminar on Language and Education, Cape Town.

Tollefsen, James. W. 1991. Planning Language, Planning Inequality. London: Longman.

Torrance, E., Gowan, J., and Alliotti, M. 1970. Creative functioning of monolingual and bilingual children in Singapore. Journal of Educational Psychology, 61: 72-75.

Tourraine, A. 1994. Qu'est-ce que la democratie. Paris: Fayard.

Urciuoli, B. 1990. The political topography of Spanish and English; the view from a New York Puerto Rican neighbourhood. American Ethnologist.

Verdoodt, A. (ed.). Applied Sociolinguistics vol. 2. AILA proceedings. Heidelberg: J. Groos Verlag.

Verspoor, A. Pathways to change. Improving the quality of education in developing countries. World Bank Discussion Papers, 53. The World Bank., Washington, D.C. 
Warman, A. 1980. "We come to object": The peasants of Morelos and the national state. Baltimore: John Hopkins Press.

Weedon, C. 1987. Feminist practice and poststructuralist theory. Oxford: Blackwell.
Weeks, Jeffrey. unpublished. The Sexual Citizen. Paper given at the Norwegian Research Council, Gender in Transition Conference 'Sexing the Self: Sexuality, Gender, Ambiguity' Oslo, 13-14 October 1997. 